\title{
THE HODGE NUMBERS OF O'GRADY 10 VIA NGÔ STRINGS
}

\author{
MARK ANDREA A. DE CATALDO, ANTONIO RAPAGNETTA, GIULIA SACCÀ,
}

\begin{abstract}
We determine the Hodge numbers of the hyper-Kähler manifold known as O'Grady 10 by studying some related modular Lagrangian fibrations by means of Nĝo strings, which we introduce via a refinement of the Ngô Support Theorem.
\end{abstract}

\section{Contents}

1. Introduction 2

2. Notation and preliminaries 5

2.1. General notation and facts 5

2.2. The notion of $\delta$-regular weak abelian fibrations $\quad 7$

2.3. Weak abelian fibrations and holomorphic symplectic manifolds 9

3. The manifolds $\widetilde{M}$ and $N$ as $\delta$-regular weak abelian fibrations 13

3.1. OG10-type manifolds $\quad 13$

3.2. Pure dimension one sheaves and the manifolds $\widetilde{M}$ and $N \quad 14$

3.3. The group schemes 18

3.4. The manifolds $\widetilde{M}, N, M^{\prime}$ and $M^{\prime(2)}$ as $\delta$-regular weak abelian fibrations 20

4. The top direct image sheaf $R^{10}$ for the Lagrangian fibrations $\widetilde{M}, N \rightarrow B \quad 22$

4.1. The Decomposition Theorem for the blow up $b: \widetilde{M} \rightarrow M \quad 22$

4.2. Irreducible components of the fibers: main results 24

4.2.1. Irreducible components arising from the exceptional divisor 24

4.2.2. Irreducible components over reduced curves 24

4.2.3. Irreducible components over non-reduced curves: the statement 25

4.3. The fibers over non-reduced curves 26

4.3.1. Irreducible components over non-reduced curves: sheaves of type I 26

4.3.2. Preliminary results towards the analysis of type II 27

4.3.3. Irreducible components over non-reduced curves: sheaves of type II 28

4.4. The top degree direct image sheaves $R^{10}$ and the local systems $\mathscr{L} \quad 36$

5. The Ngô Strings of several Lagrangian fibrations 40

5.1. Some relevant strings 41

5.2. The Decomposition Theorem for the genus two universal curve $\mathcal{C}^{\prime} \rightarrow B^{\prime} \quad 42$

5.3. The Decomposition Theorem for the genus five universal curve $\mathcal{C} \rightarrow B \quad 43$

5.4. The Ngô strings for the Lagrangian fibration $p: M^{\prime} \rightarrow B^{\prime} \quad 44$

5.5. The Ngô strings for the Lagrangian fibration $p^{2}: M^{\prime} \times M^{\prime} \rightarrow B^{\prime} \times B^{\prime} \quad 45$

5.6. The Ngô strings for the Lagrangian fibration $p^{(2)}: \operatorname{Sym}^{2} M^{\prime} \rightarrow \operatorname{Sym}^{2} B^{\prime} \quad 46$

5.7. Ngô strings for the Lagrangian fibrations $\widetilde{M}, N \rightarrow B \quad 47$

6. Proofs of Theorems A, B and B' 49 
6.1. Proof of the main Theorem A 50

6.2. The main Theorem B' 52

7. Appendix: Hodge-theoretic Ngô Strings 54

References $\quad 58$

\section{INTRODUCTION}

Irreducible holomorphic symplectic manifolds are simply connected compact Kähler manifolds with a unique up to scalar holomorphic two-form, which is furthermore symplectic. They are hyper-Kähler manifolds and are one of the three building blocks for compact Kähler manifolds with trivial first Chern class [Bea-1983, Bo-1973]. In every even complex dimension, there are two series of known examples of irreducible holomorphic symplectic manifolds: those that are deformation equivalent to the Hilbert schemes of $n$-points on a K3 surface, called of $\mathrm{K} 3^{[n]}$-type, and those that are deformation equivalent to generalized Kummer varieties, called of generalized Kummer type [Bea-1983, Fu-1983]. In addition to these, there are two sporadic deformation classes occurring in dimension 6 and 10 discovered by O'Grady [O'G-1999a, O'G-1999b] as symplectic resolutions of a singular moduli spaces of sheaves on a K3 surface (in the case of the 10-dimensional example) and on an abelian surface (in the case of the 6 -dimensional example). The class of irreducible holomorphic symplectic manifolds deformation equivalent to the 10 dimensional example is denoted by $O G 10$ and manifolds in this deformation class are said to be of OG10-type. Similarly, for the 6-dimensional example.

The Betti and Hodge numbers of irreducible holomorphic symplectic manifolds of K $3^{[n]}$ type and of generalized Kummer type are known thanks to Göttsche's Formula [Go-1990, Go-So-1993] (see also [de-Mi-2000, de-Mi-2002]). The Betti and Hodge numbers of the deformation class $O G 6$ were calculated in [Mo-Ra-Sa-2018]. The purpose of this paper is to compute the Betti and Hodge numbers of the remaining known deformation class, namely OG10. Previously, the only known invariants of this class manifolds were the second Betti number $b_{2}=24$ [Ra-2008] and the Euler number $e=176,904$ [Mo-2007, Hu-La-Sa-2019]. Our first main result is the following theorem:

Theorem A. The odd Betti numbers of the ten-dimensional irreducible symplectic manifolds of OG10-type are zero and the even ones are:

$$
\begin{array}{|l|l|l|l|l|l|}
\hline b_{0}=1 & b_{2}=24 & b_{4}=300 & b_{6}=2899 & b_{8}=22150 & b_{10}=126156 . \\
\hline
\end{array}
$$

The relevant part of the Hodge diamond is as follows:

\begin{tabular}{|c|c|c|c|c|}
\hline & \multicolumn{4}{|r|}{1} \\
\hline & & & 1 & 22 \\
\hline & & 1 & 22 & 254 \\
\hline & 1 & 23 & 276 & 2299 \\
\hline 1 & 22 & 276 & 2531 & 16490 \\
\hline 22 & 254 & 2299 & 16490 & 88024. \\
\hline
\end{tabular}


We prove the theorem by comparing the cohomology of an irreducible symplectic variety of $O G 10$-type with that of an irreducible symplectic variety of $\mathrm{K} 3^{[5]}$-type. This is done by considering two Lagrangian fibered irreducible holomorphic symplectic manifolds, denoted $\widetilde{M} \rightarrow \mathbb{P}^{5}$ and $N \rightarrow \mathbb{P}^{5}$, where the first is of $O G 10$-type and the second is of $\mathrm{K} 3^{[5]}$-type. These are chosen so that over a dense open subset of the base the two fibrations are torsors over the same group scheme. In this setting, the crucial ingredient is Ngô's Support Theorem, which allows us to exploit the Lagrangian fibration structure and to express the difference between the cohomology of $\widetilde{M}$ and that of $N$ in terms of the cohomology of lower dimensional irreducible symplectic manifolds.

Let us describe the two Lagrangian fibrations. Let $(S, H)$ be a general polarized $\mathrm{K} 3$ surface of genus 2 and let $\mathcal{C} \rightarrow \mathbb{P}^{5}$ be the universal family of the genus 5 linear system $|2 H| \simeq \mathbb{P}^{5}$. The relative compactified Jacobian $M \rightarrow \mathbb{P}^{5}$ of a given even degree of the linear system $|2 H|$ is a singular projective symplectic variety. We let $\widetilde{M} \rightarrow M$ be the blowup of its singular locus (with its reduced induced structure). This is an irreducible holomorphic symplectic manifold of $O G 10$-type and $\widetilde{M} \rightarrow M$ is a symplectic resolution. If instead we consider a relative compactified Jacobian of a given odd degree, we get a smooth projective irreducible holomorphic symplectic manifold $N \rightarrow \mathbb{P}^{5}$, deformation equivalent to the Hilbert scheme of 5 points on $S$. The degree 0 relative Picard variety $P \rightarrow \mathbb{P}^{5}$ of the linear system is a group scheme over $\mathbb{P}^{5}$, and it acts naturally and fiberwise on both spaces. It is important to note that there are dense open subsets of $\mathbb{P}^{5}$ over which the fibrations are torsors over (the restriction of) the group scheme $P / \mathbb{P}^{5}$. Note that the Lagrangian fibration $\widetilde{M} \rightarrow \mathbb{P}^{5}$ is the same used by Mozgovoy in his determination [Mo-2007] of the Euler number of $O G 10$. It is a degeneration of the Intermediate Jacobian fibration [LSV-2017, Ko-La-Sa-Vo-2018], which was used in [Hu-La-Sa-2019].

The starting point of our analysis is that each of the two Lagrangian fibrations, together with the natural action of $P$, gives rise to what Ngô calls a $\delta$-regular weak Abelian fibration (see §3.4). Before showing this, we prove in Section $\S 2.3$ some general results on weak Abelian fibrations arising from certain Lagrangian fibrations.

Ngô's Support Theorem is a topological result concerning the Decomposition Theorem for $\delta$-regular weak Abelian fibrations. Roughly speaking, it states that if in the Decomposition Theorem for the fibration a subvariety of the target is the support of a direct summand of the derived direct image of the constant sheaf, then such a subvariety (called a support for the fibration) is the support of a non trivial direct summand of the top degree direct image sheaf. What makes this theorem powerful is the possibility of restricting the set of potential supports by only using the top degree direct image sheaf.

To carry out our cohomological computation, we first need to refine the statement of Ngô Support Theorem. Given a subvariety of the base that is a support for the Decomposition Theorem of a $\delta$-regular weak Abelian fibrations, the refinement consists in identifying the direct summands supported on that subvariety. We show that they can be expressed in terms of contributions coming from lower dimensional $\delta$-regular weak abelian fibrations that naturally appear in the picture. We call these direct summands Ngô Strings (see Theorem 7.0.3 and Definition 7.0.4 ). We believe this is a result of independent interest. In addition to this, we need to make sure that a given Ngô String contributes the same pure Hodge structure, regardless of which weak Abelian fibration it appears in (cf. Remark 
7.0.5). Since we are not aware of a reference, we offer some context and a sketch of proof, which involves M. Saito's mixed Hodge modules. This is done in Appendix 7.

In order to identify which subvarieties of the base appear as supports, we need to study the top-degree higher direct images for the Lagrangian fibrations $\widetilde{M} / \mathbb{P}^{5}$ and $N / \mathbb{P}^{5}$. The determination of these top degree sheaves is the subject of the key Proposition 4.4.3, which is based on the detailed analysis of the moduli spaces of semi-stable sheaves over the locus parametrizing non-reduced curves in the linear system $|2 H|$ (see $\S 4.2-4.3$ ).

In $\S 5$ we identify the Ngô Strings of the two fibrations, i.e., we determine the direct summands appearing in the Decomposition Theorem for $\widetilde{M} / \mathbb{P}^{5}$ and $N / \mathbb{P}^{5}$. This is the content of Proposition 5.7.2. Actually, this is done up to some indeterminacy which is present in both fibrations. Remarkably, these two indeterminacies cancel out when comparing the Hodge numbers of the two fibrations, and we are thus able to deduce our main Theorem A in $\S 6.1$.

The following observations are key for successfully carrying out this strategy. First of all, the full support Ngô Strings (i.e., the direct summands whose support is the whole $\mathbb{P}^{5}$ ) are nothing but natural extensions to $\mathbb{P}^{5}$ of the local systems associated to the restriction of the fibration to the smooth locus. By our choice of $\widetilde{M} / \mathbb{P}^{5}$ and $N / \mathbb{P}^{5}$, these local systems are the same for the two fibrations and thus the contribution to the cohomology of $\widetilde{M}$ and of $N$ of the full support direct summands are the same. Secondly, we show that the Ngô Strings of the two fibrations that are supported on proper subvarieties of $\mathbb{P}^{5}$ appear also in the Decomposition Theorem for certain lower-dimensional Lagrangian fibrations associated with other moduli spaces of sheaves on the same $K 3$ surface $S$. The geometry of these is considerably simpler and their Hodge numbers are known. It follows that we may express the difference between the Hodge numbers of $\widetilde{M}$ and of $N$ in terms of the (known) Hodge numbers of lower-dimensional irreducible holomorphic symplectic manifolds.

Upgrading these cohomological comparisons to the appropriate Grothendieck group, we express the "difference" between the pure Hodge structure of $\widetilde{M}$ and the pure Hodge structure of $N$ in terms of the pure Hodge structures of lower dimensional moduli spaces of sheaves on the degree 2 K3 surface $S$. Thanks to Goettsche's formula, these can in turn be expressed as functions of the Hodge structure of $S$. This gives Theorem B below, which determines the pure Hodge structure of the manifolds $\widetilde{M}$ (which define a codimension 3 locally closed subset in the moduli space of varieties of $O G 10$-type) in terms of the pure Hodge structure of $S$.

Theorem B. Let $(S, H)$ be a general polarized $K 3$ surface of genus 2 and let $\widetilde{M}$ and $N$ be the irreducible symplectic manifolds introduced above. Let $\mathbb{S}_{(-)}$be the Schur functors $[\mathrm{Fu}-\mathrm{Ha}$, Ch 6]. Let $\langle\bullet\rangle:=[-2 \bullet](-\bullet)$. By abuse of notation, for a projective manifold $X$, we denote by $X$ also the graded rational polarizable pure Hodge structure $H^{*}(X, \mathbb{Q})$. Then we have isomorphisms:

$$
\begin{aligned}
\widetilde{M}= & S^{(5)} \oplus\left[S^{(4)}\langle-1\rangle\right]^{\oplus 2} \oplus \mathbb{S}_{(2,2)}(S)\langle-1\rangle \oplus\left[S^{(3)}\langle-2\rangle\right]^{\oplus 2} \oplus \\
& \oplus\left[\mathbb{S}_{(2,1)}(S)\langle-2\rangle\right]^{\oplus 2} \oplus[S \otimes S]\langle-3\rangle \oplus\left[S^{(2)}\langle-3\rangle\right]^{\oplus 3} \oplus[S\langle-4\rangle]^{\oplus 2}
\end{aligned}
$$




$$
N=S^{(5)} \oplus\left[S^{(3)} \otimes S\right]\langle-1\rangle \oplus\left[S \otimes S^{(2)}\right]^{\oplus 2}\langle-2\rangle \oplus\left[S^{2}\right]^{\oplus 2}\langle-3\rangle \oplus S\langle-4\rangle .
$$

Note that (2) above is well-known and it is only included for completeness. Theorem $\mathrm{B}$ is here formulated only for a general genus $2 \mathrm{~K} 3$ surface. In Section 6.2 we give the slightly more general statement (Theorem $\mathrm{B}^{\prime}$ on page 52 ), as well as its proof.

After our paper appeared, the paper [G-K-L-R-2019] computed the Hodge structure of an irreducible holomorphic symplectic variety of OG10-type (in fact, the cohomology is even determined as a module over the so-called Loojenga-Lunts-Verbitsky algebra [Lo-Lu-1997, Ve-96]), conditionally to assuming that the odd Betti numbers are zero. Then the paper [Fl-Fu-Zh-2019] appeared, where it is shown that odd Betti numbers of OG10 are trivial. Our methods and those of [G-K-L-R-2019] are completely different and we view the two papers as complementary.

Acknowledgments. We would like to thank E. Arbarello, K. Hulek, J. de Jong, R. Laza, E. Markman, L. Maxim, M. McLean, A. Toth, D. Varolin for conversations related to the topic of this paper, as well as I. Grosse-Brauckmann for pointing out several typos. M. A. de Cataldo, who has been partially supported by NSF grants DMS 1600515 and 1901975, would like to thank the Max Planck Institute for Mathematics in Bonn and the Freiburg Research Institute for Advanced Studies for the perfect working conditions; the research leading to these results has received funding from the People Programme (Marie Curie Actions) of the European Union's Seventh Framework Programme (FP7/2007-2013) under REA grant agreement n. [609305]. A. Rapagnetta acknowledges the MIUR Excellence Department Project awarded to the Department of Mathematics, University of Rome Tor Vergata, CUP E83C18000100006. G. Saccà acknowledges partial support from NSF grant DMS-1801818. We would also like to thank the anonymous referee for many suggestions that have greatly improved the exposition of the paper.

\section{Notation AND PRELIMINARIES}

\subsection{General notation and facts.}

We work over the field of complex numbers. A variety is a separated scheme of finite type over $\mathbb{C}$. Unless otherwise stated, by point we mean a closed point.

We work with the following two categories attached to a variety $S$ : the constructible bounded derived category $D^{b}(S, \mathbb{Q})$, whose objects are bounded complexes of sheaves on $S$ of rational vector spaces whose cohomology complexes are constructible with algebraic strata (see [de-Mi-2009] for the basics and references); M. Saito's bounded derived category $D^{b} M H M_{a l g}(S)$ of algebraic mixed Hodge modules with rational structure, which is endowed with the formalism of weights; see [Sai-90] for the foundations, and [Sch-14] especially $\S 22$ and $\S 23$, which contains the basics and references. We call the objects of $D^{b} M H M_{a l g}(S)$ simply "objects."

If in Theorem A one is interested only in Betti numbers, then it is enough to work with $D^{b}(-, \mathbb{Q})$. If one is interested in the Hodge numbers, then it seems necessary to work with $D^{b} M H M_{a l g}(-)$. 
There is the natural functor rat $: D^{b} M H M_{a l g}(S) \rightarrow D^{b}(S, \mathbb{Q})$. The functor rat is compatible with the usual operations (push-forwards, pull-backs, tensor, hom, duality, vanishing/nearby cycles). Via the functor rat, the standard $t$-structure on the domain corresponds to the middle perversity $t$-structure on the target (see [de-Mi-2009] for the basics on middle perversity and references); in particular, if $K \in M H M_{a l g}(S)$, then $\operatorname{rat}(K)$ is a perverse sheaf on $S$. There is a second $t$-structure on $D^{b} M H M_{a l g}(S)$ which corresponds to the standard $t$-structure in $D^{b}(S, \mathbb{Q})$ via the functor rat; [Sai-90, Remark 4.6.2]. In particular, given a morphism $f: X \rightarrow Y$ of varieties, one promotes direct image cohomology shaves $R^{i} f_{*} K, R^{i} f_{!} K$ of a complex $K$ in the essential image of rat to objects in $D^{b} M H M_{a l g}(S)$; by restricting such objects to suitably Zariski dense open subvarieties of the regular locus of their support, these objects are admissible polarizable variations of mixed Hodge structures.

The functor rat is neither essentially surjective, nor fully faithful; what is important in this paper is the evident fact that a splitting of an object $K$ induces a splitting of $\operatorname{rat}(K)$. By abuse of notation and for simplicity, we do not distinguish notationally between an object $K$ and its counterpart $\operatorname{rat}(K)$.

If $Z \subseteq S$ is a closed irreducible subvariety and $L$ is a polarizable variation of rational pure Hodge structures of some weight $w(L)$ on some Zariski-dense open subset $V$ contained in $Z^{\text {reg }}$, then the intersection cohomology object $I C_{Z}(L) \in M H M_{a l g}(S)$ yields, via rat, the usual intersection cohomology complex of $Z$ with coefficients the local system underlying $L$; such a local system must be self-dual, i.e. $L \simeq L^{\vee}(-w(L))$, and semi-simple, i.e. $L$ splits into a direct sum of simple objects. One can shrink $V$, if desired; this is useful when one desires $V$ to be subject to certain conditions.

The objects of $M H M_{a l g}(S)$ which are pure of weight $w$ are exactly the ones of the aforementioned form $I C_{Z}(L)$, with $L$ pure of weight $w(L)=w-\operatorname{dim} Z$; they are semisimple, and every simple object has this form with, $L$ simple as polarizable variation of pure Hodge structures. Note that the corresponding object $\mathscr{I} \mathscr{C}_{Z}(L):=I C_{Z}(L)[-\operatorname{dim} Z]$ in $M H M_{a l g}(S)[-\operatorname{dim} Z]$ in $D^{b} M H M_{a l g}(S)$ is pure of weight $w-\operatorname{dim} Z$; the cohomology sheaves of $\operatorname{rat}\left(\mathscr{I C}_{Z}(L)\right)$ are in non-negative degrees, starting at zero. For the geometric statements, e.g. (64), we use $\mathscr{I} \mathscr{C}$, and at times, in order to exploit the simplified bookkeeping stemming from Poincaré-Verdier and Relative Hard Lefschetz dualities, we use $I C$, e.g. (65).

Let $Z$ be irreducible and complete and let $L$ (on some $V \subseteq Z^{\text {reg }} \subseteq Z$ ) be as above, pure of weight $w(L)$. Then the cohomology groups $I H^{\bullet}(Z, L):=H^{\bullet}\left(Z, \mathscr{I} \mathscr{C}_{Z}(L)\right)$ are polarizable pure Hodge structures of weight $w(L)+\bullet$. Polarizations are typically not unique, but the pure Hodge structures on $I H^{\bullet}(Z, L)$ do not depend on a choice of polarization. The category of rational polarizable pure Hodge structures is abelian semisimple: every subobject admits a direct sum complement; the induced splitting does not need to preserve given polarizations.

An object $L$ as above of weight $2 v \in \mathbb{Z}^{\text {ev }}$ is said to be of pure Hodge-Tate type $(v, v)$ if the pure Hodge structure on the stalks is of type $(v, v)$. If the polarizable variation of pure Hodge structures $L$ is of pure Hodge-Tate type and of weight $2 v$, then the underlying local system determines the structure of polarizable variation of pure Hodge structure of weight $2 v$ on $L$ uniquely, i.e. if $L, L^{\prime}$ are two polarizable variations of pure Hodge structures, 
which are both pure of Hodge-Tate type and of the same weight $2 v$, and such that the underlying local systems are isomorphic, then $L \simeq L^{\prime}$ as objects in $M H M_{a l g}(S)$; this is simply because the associated Hodge filtration is trivial.

Let $f: X \rightarrow Y$ be a proper morphism of varieties and let $K \in M H M_{\text {alg }}(X)$ be pure of weight $w$. E.g. $K=I C_{X}$, or even $K=\mathbb{Q}_{X}[\operatorname{dim} X]$ when $X$ is irreducible nonsingular; in either case, $w=\operatorname{dim} X$. In this context, the Decomposition and the Relative Hard Lefschetz Theorems (see [de-Mi-2009] for references) give : $R f_{*} K \in D^{b} M H M_{a l g}(Y)$ is pure of weight $w$, it splits as the direct sum $\oplus_{b}{ }^{p} H^{b}\left(R f_{*} K\right)[-b]$ of its shifted perverse cohomology sheaves, and each ${ }^{p} H^{b}\left(R f_{*} K\right)[-b]$ is pure semisimple of weight $w-b$; if in addition, $f$ is projective and $\mathfrak{l}$ is the first Chern class of an ample line bundle on $X$, then cupping with the powers of $\mathfrak{l}$ induces the Relative Hard Lefschetz isomorphism $\mathfrak{r}^{k}:{ }^{p} H^{-k}\left(R f_{*} K\right) \simeq{ }^{p} H^{k}\left(R f_{*} K\right), \forall k \geq 0$. Of course, similar statements hold for a shifted $K[a]$, so that, after the evident bookkeeping, they apply, for example, to $\mathscr{I}_{\mathscr{C}_{X}}$ (this is defined for not necessarily irreducible nor pure dimensional varieties and is pure of weight zero as the direct sum of the $\mathscr{I} \mathscr{C}$ 's of the irreducible components (cf. [de-2012, §5]).

Similar consideration hold for $R f_{*} \mathscr{I}_{\mathscr{C}_{X}}$ in $D^{b}(X, \mathbb{Q})$. A statement in $D^{b} M H M_{\text {alg }}(-)$ may contain Tate shifts; when considering the resulting statement in $D^{b}(-, \mathbb{Q})$ it is understood that one omits the meaningless Tate shifts.

If $f: X \rightarrow Y$ and $K$ are as above, then the closed subvarieties that are the supports of a simple summand of the direct image $R f_{*} \mathscr{I}_{\mathscr{C}}$ are called the supports of $f$. The notion of support is of paramount importance in this paper. The determination of the supports of a morphism is a subtle problem. Of course, one can define the supports of $R f_{*} K$ for any object, by taking the supports of the constituents of $R f_{*} K$, which is the unique finite collection of simple perverse sheaves appearing in a Jordan-Hölder decomposition of the perverse sheaves ${ }^{p} H^{k}\left(R f_{*} K\right)$ for $k \in \mathbb{Z}$.

Let $f: X \rightarrow Y$ be a morphism, let $Z \subseteq Y$ be a subvariety. Then we set $X_{Z}:=f^{-1}(Z)$. In particular, if $y \in Y$ is a point, then $X_{y}=f^{-1}(y)$ denotes the fiber.

2.2. The notion of $\delta$-regular weak abelian fibrations. In [Ngô-2010, Ngô-2011] B.C. Ngô introduced the notion of $\delta$-regular weak abelian fibration, which is a notion of paramount importance in our approach to the proof of Theorem A. For more details on what follows, we refer to the papers of Ngô quoted above as well as to our Appendix §7, which contains the refinement of Ngô Support Theorem needed in this paper.

Let $g: P \rightarrow S$ be a smooth commutative group $S$-scheme, and let $g^{o}: P^{o} \rightarrow S$ be its identity component. Given any Zariski point $s$ in $S$, there is the canonical Chevalley devissage of the fiber $P_{s}^{o}$ of $P$ at $s$, i.e. a canonical short exact sequence of connected commutative group schemes $0 \rightarrow R_{s} \rightarrow P_{s}^{o} \rightarrow A_{s} \rightarrow 0$ over the perfect field $k(s)$, where $R_{s}$ is affine and connected, and maximal with these properties, and where $A_{s}$ is an abelian variety (cf. e.g.: [Mi-2012, Thm 10.25, Prop 10.24, Prop 10.5 (and its proof), Prop 10.3].

We introduce the quantity $\delta(s):=\operatorname{dim}_{k(s)} R_{s}$, which plays an important role in this paper. For example, if $P_{s}$ is the Jacobian of a reduced projective curve $C$, then $\delta$ is the dimension of $\operatorname{ker}\left[\nu^{*}: \operatorname{Pic}(C) \rightarrow \operatorname{Pic}(\hat{C})\right]=\left(\nu_{*} \mathcal{O}_{\hat{C}}^{\times} / \mathcal{O}_{C}^{\times}\right) / \operatorname{Im} H^{0}\left(\hat{C}, \mathcal{O}_{\hat{C}}^{\times}\right)$where $\nu: \hat{C} \rightarrow C$ is the normalization morphism (cf. [EGA IV(4), 21.8.5]). If $C$ is integral with one node or cusp and no other singular point, then $\delta=1$; if $C$ is a curve with two nodes and no 
other singularities, then $\delta=2$ when $C$ is irreducible, and $\delta=1$ when $C$ is the union of two irreducible components. The function $\delta$ is upper-semicontinuous on $S$. If $Z \subseteq S$ is an irreducible subvariety, then one sets $\delta(Z)$ to be the value $\delta\left(\eta_{Z}\right)$ at the generic point $\eta_{Z}$ of $Z$. In this case, we have that $\delta(Z)$ coincides with the value $\delta(z)$ at a general point $z \in Z$, and also with the minimum of $\delta(-)$ on the points of $Z$. A crucial role is played by the $\delta$-loci, which are the locally closed subvarieties $S_{i} \subseteq S$ defined by setting: $S_{i}:=\{s \in S \mid \delta(s)=i\}$. Let $d:=\operatorname{dim} P / S$ be constant (it is so on the connected components of $S$ ). The Tate module $P / S$ is the object $T(P)=T(P / S):=R^{2 d-1} g_{!}^{o} \mathbb{Q}_{P^{o}}(d)$ in $D^{b} M H M(S)_{a l g}$ (cf. [Ngô-2010, §4.12]; the Tate shift $(d)$ is in the sense of M. Saito's theory of mixed Hodge modules (cf. §2.1). Given a point $s$ of $S$, we have the short exact sequence $0 \rightarrow T\left(R_{s}\right) \rightarrow T\left(P_{s}^{o}\right) \rightarrow T\left(A_{s}\right) \rightarrow 0$ of rational mixed Hodge structures. Note that $\operatorname{dim} T\left(R_{s}\right) \leq \delta(s)$ and the strict inequality is possible, e.g. a projective rational curve with a cusp.

For a fixed prime $\ell$, let $T_{\text {et, }}(P / S)$ be the $\overline{\mathbb{Q}}_{\ell}$-adic counterpart to $T(P / S)$ (defined by the analogous formula, using the étale topology/cohomology formalism). We say that $T_{\text {et, } \overline{\mathbb{Q}}_{\ell}}(P / S)$ is polarizable if étale-locally there is a pairing $T_{\text {et, }, \overline{\mathbb{Q}}_{\ell}}(P / S) \otimes T_{\text {et, }, \overline{\mathbb{Q}}_{\ell}}(P / S) \rightarrow$ $\overline{\mathbb{Q}}_{\ell}(1)$ such that, for every point $s$ of $S$, the null space of the pairing at $s$ is precisely $T_{\text {et, } \overline{\mathbb{Q}}_{\ell}}\left(R_{s}\right)$.

Definition 2.2.1. ( $\delta$-regular weak abelian fibration) $A$ weak abelian fibration is a pair of morphisms: $(f: M \rightarrow S, g: P \rightarrow S)$ such that:

(a) $f$ is proper;

(b) $P$ is a smooth commutative group scheme;

(c) There is an action $a: P \times_{S} M \rightarrow M$ of $P$ on $M$ over $S$;

(d) $f$ and $g$ have the same pure relative dimension, denoted by $d$;

(e) the action has affine stabilizers at every point $m \in M$;

(f) the Tate module $T_{\text {et, } \overline{\mathbb{Q}}_{\ell}}(P / S)$ of $P / S$ is polarizable. In context, we abbreviate $(f$ : $M \rightarrow S, g: P \rightarrow S)$ to $(M, S, P)$.

If, in addition, we also have the following property of $P / S$ :

$$
\operatorname{codim} S_{i} \geq i, \quad \forall i \in \mathbb{Z}^{\geq 0},
$$

then we say that $(M, S, P)$ is a $\delta$-regular weak abelian fibration.

Remark 2.2.2. Note that the $\delta$-regularity inequality (3) is equivalent to:

$$
\operatorname{codim}_{S} Z \geq \delta(Z), \quad \text { for every locally closed integral subvariety } Z \subseteq S .
$$

Moreover, (3) implies that if $(M, S, P)$ is a $\delta$-regular weak abelian fibration, then the general fiber $P_{s}^{o}$ is complete, i.e. it is an Abelian variety.

If $P \rightarrow S$ is the relative degree- 0 Picard scheme of a family of integral curves parametrized by $S$, then the fibration is $\delta$-regular if and only if for all $\delta \geq 0$, the locus of curves of cogenus $\delta$ has codimension $\geq \delta$. Examples of $\delta$-regular weak abelian fibrations will be given in Example 2.3.3 and in $§ 3.3$.

Over a perfect field the Chevalley devissage exists and is unique. Since we are working in characteristic zero, by working with Zariski points on $S$, one sees that (cf. [Ngô-2010, 
$\S 7.4 .8]$ ), given any integral locally closed subvariety $Z \subseteq S$, there exists an open and dense subvariety $V \subseteq Z$ and a short exact sequence of smooth commutative group schemes over $V$ with connected fibers:

$$
0 \rightarrow R_{V} \rightarrow P_{\mid V}^{o} \rightarrow A_{V} \rightarrow 0
$$

that realizes the Chevalley devissage point-by-point on $V$. One can shrink $V$, if needed.

\subsection{Weak abelian fibrations and holomorphic symplectic manifolds.}

In this section we consider weak abelian fibrations arising from Lagrangian fibrations and we show that under some assumptions they are $\delta$-regular (Proposition 2.3.2). Examples of such Lagrangian fibrations are given in Example 2.3.3 and §3.

In this section, we fix the following data: let $\left(M, \sigma_{M}\right)$ be a quasi-projective holomorphic symplectic manifold of dimension $2 d$ with holomorphic symplectic form $\sigma_{M}$; let $S$ be a smooth variety of dimension $d$; let $f: M \rightarrow S$ be a proper Lagrangian fibration, i.e. $f$ is a proper surjective morphism with connected fibers whose general fiber is a Lagrangian subvariety; let $P / S$ be a smooth commutative group scheme with connected fibers, acting on $M / S$.

By [Ma-2000, Thm 1], every irreducible component of every closed fiber of $f$ is Lagrangian. This means that for every $s \in S$, the pullback of the symplectic form to a resolution of the singularities of any component of the fiber $M_{s}$, endowed with the reduced structure, is trivial. In particular, $f$ is equidimensional. By [Bea-1999, Prop 1], the morphism $f$ is an algebraic completely integrable system. It follows that the general fiber of $f$ is a compact complex torus.

As we show in Lemma 2.3.1, the fact that $M \rightarrow S$ is a Lagrangian fibration has consequences on the action of $P / S$ on $M$. This Lemma is then used to prove the $\delta$-regularity property. The action of the group scheme $P / S$ on the Lagrangian fibration $M / S$ defines a commutative diagram with Cartesian squares:

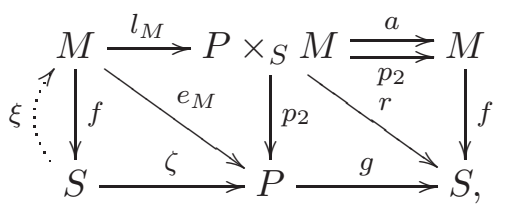

where $\zeta: s \mapsto e_{s} \in P_{s}$ (the identity section), $l_{M}: M_{s} \ni m_{s} \mapsto\left(e_{s}, m_{s}\right), a$ is the action, $p_{i}$ the projections. Note that $e_{M}\left(m_{s}\right)=e_{s}$. The dotted arrow $\xi$ is not part of the initial data, but is produced, after a suitable base change, under the hypotheses of Lemma 2.3.1.(2a).

Lemma 2.3.1. (Duality for the action) Let $M / S, \sigma_{M}, P / S$ and $a_{M}$ be as above. 
(1) There is a commutative diagram:

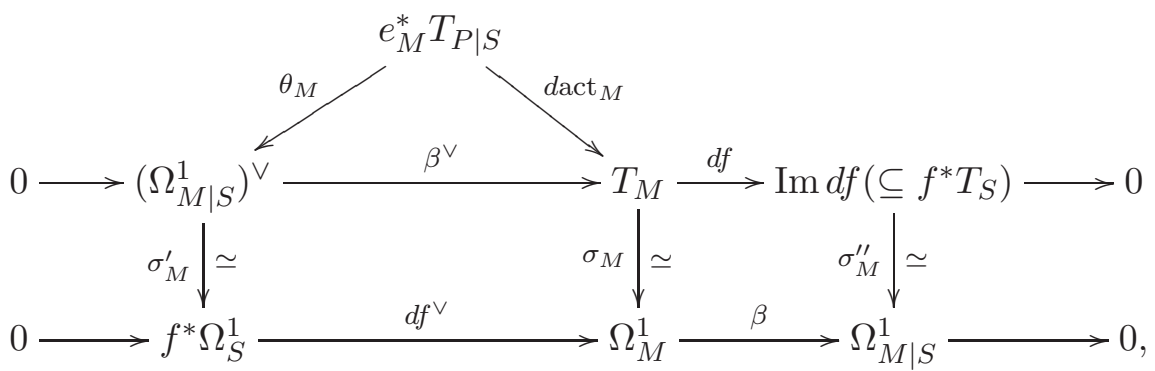

where: $e_{M}^{*} T_{P \mid S}=f^{*} \zeta^{*} T_{P \mid S}$ is the pull-back via $f$ of the vector bundle on $S$ with fibers the Lie algebras $\operatorname{Lie}\left(P_{s}\right) ; \Omega_{M \mid S}^{1}$ is torsion free; $\operatorname{Ker} d f=\left(\Omega_{M \mid S}^{1}\right)^{\vee}=$ $\mathcal{H}_{\text {om }} \mathcal{O}_{M}\left(\Omega_{M \mid S}^{1}, \mathcal{O}_{M}\right)$ is locally free; the vertical arrows are isomorphisms induced by the symplectic form $\sigma_{M}$; rows two and three are short exact sequences.

The formation of the diagram is compatible with étale base change $S^{\prime} \rightarrow S$ and with restriction to non-empty, not-necessarily P-invariant, open subsets of $M$. In particular, $\theta_{M}$ is an isomorphism if an only if it is an isomorphism after base change $S^{\prime} \rightarrow S$ given by an étale covering. The roof of the diagram depends on all the initial data, while the rest of the diagram depends only on $M / S$ and $\sigma_{M}$.

(2) Assume in addition that:

(a) either there is an open subset $M^{\circ} \subseteq M$ such that $M^{\circ} / S$ is a $P / S$-torsor;

(b) or there are: an open subset $S^{\natural} \subseteq S$, with $\operatorname{codim}\left(S \backslash S^{\natural}\right) \geq 2$, and an open subset $M^{\natural \circ} \subseteq M^{\natural}$ such that $M^{\natural \circ} / S^{\natural}$ is a $P^{\natural} / S^{\natural}$-torsor.

Then $\theta_{M}$ is an isomorphism. In particular, dact ${ }_{M}$ and $d f$ are dual to each other via the symplectic form $\sigma_{M}$, i.e., for every $m \in M$ with $s:=f(m)$, we have the commutative diagram at the level of fibers at $m$ :

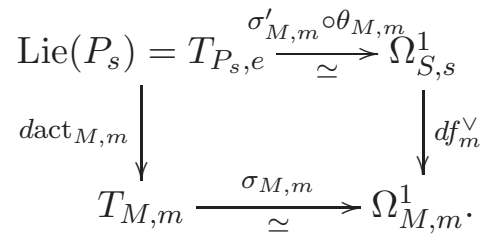

Proof. We start by explaining the isomorphism of the two horizontal short exact sequences in (7). Consider the short exact sequence $0 \rightarrow f^{*} \Omega_{S}^{1} \stackrel{d f^{\vee}}{\rightarrow} \Omega_{M}^{1} \stackrel{\beta}{\rightarrow} \Omega_{M \mid S}^{1} \rightarrow 0$. The first morphism is injective because it is generically injective ( $f$ is a dominant generically smooth morphism) and $f^{*} \Omega_{S}^{1}$ is locally free. Dualizing, we get a short exact sequence $0 \rightarrow\left(\Omega_{M \mid S}^{1}\right)^{\vee} \stackrel{\beta^{\vee}}{\rightarrow} T_{M} \stackrel{d f}{\rightarrow} \operatorname{Im} d f \rightarrow 0$, where $\operatorname{Im}\left[d f: T_{M} \rightarrow f^{*} T_{S}\right] \subset f^{*} T_{S}$. Since $f$ is Lagrangian, the composition $\beta \sigma_{M} \beta^{\vee}$ is zero, because it is generically zero and hence zero by torsion-freeness of the source. This gives, uniquely, an injective $\sigma_{M}^{\prime}$ and a surjective $\sigma_{M}^{\prime \prime}$ making the diagram commutative. Since $\sigma_{M}^{\prime \prime}$ is a surjective morphism of coherent sheaves of the same rank, it is also generically injective. By the torsion-freeness of its source $\sigma_{M}^{\prime \prime}$ 
is injective and thus an isomorphism. At this juncture, $\sigma_{M}^{\prime}$ must be an isomorphism as well.

Now we construct the roof of (7). The action $a$ induces a morphism $a^{*} \Omega_{M \mid S}^{1} \stackrel{d a^{\vee}}{\rightarrow}$ $\Omega_{P{ }_{S} M \mid S}^{1}=p_{1}^{*} \Omega_{P \mid S}^{1} \oplus p_{2}^{*} \Omega_{M \mid S}^{1}$. Precomposing with $a^{*} \beta: a^{*} \Omega_{M}^{1} \rightarrow a^{*} \Omega_{M \mid S}^{1}$ and postcomposing with the projection proj : $\Omega_{\mathrm{P} \times{ }_{\mathrm{S}} \mathrm{M} \mid \mathrm{S}}^{1} \rightarrow \mathrm{p}_{1}^{*} \Omega_{\mathrm{P} \mid \mathrm{S}}^{1}$ yields a morphism of vector bundles $a^{*} \Omega_{M}^{1} \rightarrow p_{1}^{*} \Omega_{P \mid S}^{1}$, which we dualize: this gives a morphism $p_{1}^{*} T_{P \mid S} \rightarrow a^{*} T_{M}$, which we pullback via $l_{M}^{*}$. We obtain a morphism of vector bundles

$$
d \operatorname{act}_{M}: l_{M}^{*} p_{1}^{*} T_{P \mid S}=e_{M}^{*} T_{P \mid S} \rightarrow l_{M}^{*} a^{*} T_{M}=T_{M}
$$

Notice that $e_{M}^{*} T_{P \mid S}$ is the pull-back via $f$ of the vector bundle on $S$ with fibers the Lie algebras of the fibers of $P / S$. Notice also that the morphism $d$ act $_{M}$ deserves its name: by construction, for every $s \in S$ and every $m \in M$ with $m$ over $s$, the fiber $\left(e_{M}^{*} T_{P \mid S}\right)_{m}=\operatorname{Lie} P_{s}$, and $d \operatorname{act}_{m}(v)=d a_{m}(v)$, where $a_{m}: P_{s} \rightarrow M_{s} \subset M$ sends $p \in P_{s}$ to $p \cdot m$ (where $\cdot$ denotes the action of $P / S$ on $M / S$ ).

Note that $a^{*}$ and duality commute by the smoothness of $a$. By construction, $d \operatorname{act}_{M}$ factors as $e_{M}^{*} T_{P \mid S} \longrightarrow l_{M}^{*} a^{*}\left(\Omega_{M \mid S}^{1}\right)^{\vee}=\left(\Omega_{M \mid S}^{1}\right)^{\vee} \stackrel{\beta^{\vee}}{\longrightarrow} T_{M}$, where the first morphism, which we denote by $\theta_{M}$, is $l_{M}^{*}\left(\operatorname{proj} \circ d a^{\vee}\right)^{\vee}: e_{M}^{*} T_{P \mid S} \longrightarrow\left(\Omega_{M \mid S}^{1}\right)^{\vee}$.

Let us also note the following. Assume that the general stabilizer of the action of $P / S$ on $M / S$ is trivial. Then the morphism $d$ act $_{M}$, and thus $\theta_{M}$, is injective. If $U \subseteq M$ is a $P$-invariant Zariski-dense open subset with the property that the stabilizers of its points are trivial, then the restriction $\theta_{U}$ is an isomorphism, for the action of $P$ is free on $U$.

Let us prove part (2a). There is an étale covering of finite type $S^{\prime} \rightarrow S$ such that (we denote, temporarily, objects after base change with a prime) the torsor $\left(M^{\circ}\right)^{\prime} / S^{\prime}$ admits a section $\xi: S^{\prime} \rightarrow\left(M^{\circ}\right)^{\prime}$. It is enough to show that $\theta_{M^{\prime}}$ is an isomorphism. We may thus assume that the original $M^{\circ}$ is a trivializable $P / S$-torsor, and we drop the primes. Since our aim is to verify that $\theta_{M}$ is an isomorphism, we may assume that $M$ is irreducible. Let $\tau: P \stackrel{\sim}{\rightarrow} M^{\circ}$ be the corresponding trivialization: $P_{s} \ni p \mapsto p \cdot \xi(s)$. The structural $S$-morphism of $P / S$ is Lagrangian for the symplectic form $\sigma_{P, \tau}:=\tau^{*} \sigma_{M^{\circ}}$, so that we have the standard natural isomorphism $u_{\sigma_{P, \tau}}: \Omega_{S}^{1} \stackrel{\sim}{\rightarrow} \zeta_{P}^{*} T_{P \mid S}$, with pull-back $g^{*} u_{\sigma_{P, \tau}}: g^{*} \Omega_{S}^{1} \stackrel{\sim}{\rightarrow} g^{*} \zeta_{P}^{*} T_{P \mid S}=e_{P}^{*} T_{P \mid S}$ given by $\theta_{P}^{-1} \circ\left(\sigma_{P, \tau}^{\prime}\right)^{-1}$. Pulling-back via $f$, gives the isomorphism: $f^{*} u_{\sigma_{P, \tau}}: f^{*} \Omega_{S}^{1} \stackrel{\sim}{\rightarrow} f^{*} \zeta_{P}^{*} T_{P \mid S}=e_{M}^{*} T_{P \mid S}$.

CLAIM: $u_{M, \tau}:=\sigma_{M}^{\prime} \circ \theta_{M} \circ f^{*} u_{\sigma_{P, \tau}} \in \operatorname{End}\left(f^{*} \Omega_{S}^{1}\right)$ is an isomorphism, so that, since the first and third factors are isomorphisms, $\theta_{M}$ is an isomorphism, and (2a) follows.

Proof of the CLAIM (and end of proof (2a)). Note that the section $\xi$ of $M^{\circ} / S$ also defines a section, denoted by the same symbol, of $M / S$, i.e. of $f$. We now have diagram (6) in its entirety. Using the adjoint pair $\left(f^{*}, f_{*}\right)$, that $f \circ \xi=\operatorname{Id}_{S}$, the projection formula, and the fact that $f_{*} \mathcal{O}_{M}=\mathcal{O}_{S}$ ( $f$ is its own Stein factorization), we see that the adjunction isomorphism $\operatorname{Hom}\left(f^{*} \Omega_{S}^{1}, f^{*} \Omega_{S}^{1}\right) \stackrel{\sim}{\rightarrow} \operatorname{Hom}\left(\Omega_{S}^{1}, \Omega_{S}^{1}\right)$ coincides with $\xi^{*}$, and has inverse $f^{*}$ : a morphism $\varphi$ in the former group is the pull-back via $f$ of a unique morphism in the latter, namely $\xi^{*} \varphi$, and, moreover, $\varphi$ is an isomorphism if and only if $\xi^{*} \varphi$ is an isomorphism. It remains to show that $\xi^{*} u_{M, \tau}$ is an isomorphism. Since the first and last factors are 
isomorphisms, it remains to show that $\xi^{*} \theta_{M}$ is an isomorphism. This is automatic, since $\xi(S)$ is inside the $P$-torsor $M^{\circ}$ and, as it has been observed above, $\theta_{M^{\circ}}$ is an isomorphism.

Let us prove part (2b). Note that (2b) implies (2a), however, for clarity, we have chosen to prove (2a) first. We apply (2a) to the situation of $S^{\natural}$. We obtain that the morphism $\theta_{M}$ of vector bundles on $M$ is an isomorphism in codimension two, hence an isomorphism.

In the next proposition, by abuse of notation, when we write something like $T_{P_{s}, e}$, etc., we mean the fiber of the corresponding vector bundle, not the stalk of the coherent sheaf.

Proposition 2.3.2. ( $\delta$-regularity) Let $(M, S, P)$ be a weak abelian fibration such that: $M$ and $S$ are nonsingular; $M$ is quasiprojective; $M / S$ is a proper Lagrangian fibration; $P / S$ has connected fibers; there is an open subset $M^{\circ} \subseteq M$ such that $M^{\circ} / S$ is a $P / S$ torsor. Then $(M, S, P)$ is $\delta$-regular (cf. (4)).

Proof. Let $Z \subset S$ be a locally closed integral subvariety. By shrinking $Z$ if necessary, we have the Chevalley devissage (5) $0 \rightarrow R_{Z} \rightarrow P_{Z}^{o} \rightarrow A_{Z} \rightarrow 0$ of the identity component $P_{Z}^{o}$ in affine and Abelian parts. For a general point $z \in Z, \operatorname{dim} A_{z}=\operatorname{dim} S-\delta(Z)$.

It is enough to show that for a general point $z \in Z$, we have a surjection:

$$
T_{A_{z}, e} \rightarrow \Omega_{Z, z}^{1},
$$

since this implies that $d-\delta(Z) \geq \operatorname{dim} Z$, which is our contention (4).

It is enough to obtain a surjection (9) after an étale base change $Z^{\prime} \rightarrow Z$ with $Z^{\prime}$ integral.

Let $M_{Z} \rightarrow Z$ be the restriction of $M \rightarrow S$ over $Z$. Let $\eta_{Z} \in Z$ be the generic point. Since $f$ is proper, $M_{\eta_{Z}} / \eta_{Z}$ is complete.

Let $L / k\left(\eta_{Z}\right)$ be a finite field extension so that $M_{L} / L$ has an $L$-rational point and such that the affine solvable $R_{L}$ is $L$-split. By the Borel fixed-point theorem [Bo-1991, $\S \mathrm{V}$, Proposition 15.2], $M_{L}$ admits an $L$-rational point that is fixed by $R_{L}$. By taking the integral closure $Z^{\prime}$ of $Z$ in $L$, and after shrinking $Z^{\prime}$ if necessary, we obtain a morphism $Z^{\prime} \rightarrow Z$ such that the resulting $M_{Z^{\prime}} \rightarrow Z^{\prime}$ admits a section -corresponding to the fixed $L$-rational point found above- fixed by $R_{Z^{\prime}}$, and such that $Z^{\prime} \rightarrow Z$ is étale. Let $Q_{Z}^{\prime}$ be the image of this section.

In view of our objective (9), we may now assume without loss of generality that $Z=Z^{\prime}$.

The action of $R_{Z}$ is trivial on $Q_{Z}$. If $m \in Q_{Z}$, and $z \in Z$ is the point over which $m$ lies, then the infinitesimal action of $P$ on $M$ at $m$ factors as follows: $\operatorname{Lie}\left(P_{z}\right) \rightarrow \operatorname{Lie}\left(A_{z}\right) \rightarrow$ $T_{M, m}$. The compositions $Q_{Z} \rightarrow Z \rightarrow S$ and $Q_{Z} \rightarrow M \rightarrow S$ coincide. By combining this coincidence of compositions, with the factorization of the infinitesimal actions, and with (8), we obtain the commutative diagram:

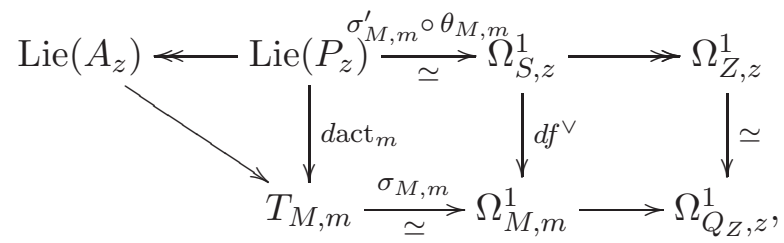

It follows that the image of $T_{A_{z}, e}$ in $\Omega_{Q_{Z}, z}^{1}$ coincides with the injective image of $\Omega_{Z, z}^{1}$. Hence the desired surjection (9) at a general point of $Z$. 
Example 2.3.3. Let $(S, H)$ be a general polarized $K 3$ surface. Let $\chi$ be an integer, set $v=(0, H, \chi)$, and let $\pi: M_{v}(S) \rightarrow|H|$ be the moduli space of pure dimension 1 sheaves on $S$ with Mukai vector $v$ (see §3.2). This is a smooth projective irreducible holomorphic symplectic manifold. Let $P \rightarrow|H|$ be the relative degree-0 Picard scheme of the family of curves in $|H|$, which exists as a scheme since the curves in $|H|$ are reduced an irreducible ([Bo-Lu-Ra-1990, Thm 1 §8.2]). Then $P /|H|$ acts on $M_{v}(S) /|H|$ with affine stabilizers (see the forthcoming Lemma 3.4.1 and 3.4.4), the Tate modules is polarizable (cf. Lemma 3.4.5) so the pair $\left(M_{v}(S), P\right)$ is a weak abelian fibration. Over the open subset $\mathcal{U} \subset M_{v}(S)$ parametrizing line bundles on the curves of $|H|$, the action is free. Since the fibers of $\pi$ are compactifed Jacobians of locally planar integral curves, the assumption of Proposition 2.3.2 are satisfied (with $M^{\circ}=\mathcal{U}$ ) and the fibration is $\delta$-regular.

For more details on the background and context for this example and for other weak abelian fibrations see $\S 3.3$.

\section{The Manifolds $\widetilde{M}$ And $N$ as $\delta$-Regular Weak abelian Fibrations}

In this section we introduce the Lagrangian fibered irreducible holomorphic symplectic manifolds $\widetilde{M}$ and $N$, as well as other auxiliary fibrations. We start by assembling some known facts about $O G 10$-type varieties (§3.1) and about moduli spaces of pure dimension one sheaves (§3.2) that are needed in the paper. Then we show that the Lagrangian fibrations we have introduced are $\delta$-regular weak abelian fibrations ( $§ 3.3$ and 3.4).

3.1. OG10-type manifolds. Let $(S, H)$ be a polarized K3 surface with Néron-Severi group $N S(S)=\mathbb{Z} H$. We identify vectors $v:=\left(v_{0}, v_{2}, v_{4}\right) \in \mathbb{Z}^{3}$ with elements in $H_{\text {alg }}^{\text {even }}(S, \mathbb{Z})$, via the obvious identification, and we consider the even quadratic form $v^{2}:=v_{2}^{2} H^{2}-2 v_{0} v_{4}$ on $H_{\text {alg }}^{\text {even }}(S, \mathbb{Z})$ induced by the Mukai pairing. A vector $v \in H_{\text {alg }}^{\text {even }}(S, \mathbb{Z})$ is called positive (cf. [Yos-01, Def. 0.1] and [Ba-Ma-2014b, Def. 5.1]) if $v^{2} \geq-2$ and if either: $\left.a\right) v_{0}>0$; or b) $v_{0}=0, v_{2}>0$; or $\left.c\right) v_{0}=v_{2}=0$ and $v_{4}>0$. We say that a Mukai vector $v$ is primitive if $v$ is not of the form $k v^{\prime}$, for $k \neq \pm 1$. For a coherent sheaf $\mathcal{F}$ on $S$ we denote by $v(\mathcal{F}):=\operatorname{ch}(\mathcal{F}) \cup \sqrt{\operatorname{td}(\mathrm{S})}$ the Mukai vector of $\mathcal{F}$.

Remark 3.1.1. Conditions $a), b)$ and $c$ ) are necessary on $v$ for the existence of a coherent sheaf $\mathcal{F}$ with $v(\mathcal{F})=v$. In [Yos-01, Def. 0.1] and [Ba-Ma-2014b, Def. 5.1], in case b) it is assumed that $v_{4} \neq 0$ in order to ensure that for primitive $v$ and generic polarization, semistability implies stability for $\mathcal{F}$. Since this is automatic if $N S(S) \simeq \mathbb{Z}$, we drop this assumption (cf. [Pe-Ra-2017, Rmk 2.6]). However, in the few cases where we deal with K3 surfaces with higher Picard rank (Remark 3.2.3 and Theorem $B^{\prime}$ ), we refer to the notion of positivity for Mukai vectors given in [Yos-01, Def. 0.1] and [Ba-Ma-2014b, Def. 5.1]

Let $v^{\prime}$ be a primitive and positive Mukai vector, let $m \geq 1$, and set $v:=m v^{\prime}$. What follows is classical work of several authors [Mu-1984, Yos-01, Ka-Le-So-2006, O'G-1999a]. The moduli space $M_{v}(S)$ of Gieseker-semistable pure sheaves on $S$ with Mukai vector equal to $v$ is a normal irreducible projective variety of dimension $v^{2}+2$ [Gi-1977, Theorem 0.3] and [Ka-Le-So-2006, Theorem 4.4]. The points of $M_{v}(S)$ are the isomorphism classes of polystable sheaves or, equivalently, the S-equivalence classes of semistable sheaves [Hu-Le-1997, Theorem 4.3.3]. By [Mu-1984], the smooth locus of $M_{v}(S)$ is precisely the 
locus parametrizing stable sheaves and it admits a holomorphic symplectic form. It follows that the moduli space is smooth if and only if the locus parametrizing strictly semistable sheaves is empty. When $N S(S) \simeq \mathbb{Z}$, this happens if and only if $m=1$, i.e. if and only if $v$ is primitive. If this is the case, the moduli space is an irreducible holomorphic symplectic manifold deformation equivalent to the Hilbert scheme of $\frac{1}{2} v^{2}+1$ points on $S$ [Yos-01, Theorem 8.1]. For $m \geq 2$, the singular moduli space $M_{v}(S)$ admits a symplectic resolution if and only if $m=2$ and $v^{2}=2$ [O'G-1999a, Ka-Le-So-2006, Le-So-2006]. In this case, the following result holds:

Theorem 3.1.2. (OG10-type manifolds) Let $v=2 v^{\prime}$, with $v^{\prime}$ positive and $v^{\prime 2}=2$.

(1) The singular locus of $M_{2 v^{\prime}}(S)$ is naturally identified with the 8-dimensional $\operatorname{Sym}^{2} M_{v^{\prime}}(S)$.

(2) Locally in the classical topology, the singularities of $M_{2 v^{\prime}}(S)$ do not depend on the choice of $v^{\prime}$ with $v^{\prime 2}=2$. Moreover, the blow up $\widetilde{M}_{2 v^{\prime}}(S) \rightarrow M_{2 v^{\prime}}(S)$ of $M_{2 v^{\prime}}(S)$ along the singular locus, with its reduced induced structure, is a symplectic resolution.

(3) The symplectic resolution $\widetilde{M}_{v}(S)$ is an irreducible holomorphic symplectic manifold deformation equivalent to O'Grady's 10-dimensional exceptional example.

Proof. (1) Follows from the fact that since $N S(S)=\mathbb{Z}$ the polystable sheaves with Mukai vector $v$ are of form $F_{1} \oplus F_{2}$, with $v\left(F_{i}\right)=v^{\prime}$. (2) This is [Le-So-2006, Thms 1.1 and 4.5] (for the rank 2 case, see also [Ka-Le-2007, Prop 2.2]). (3) This is [Pe-Ra-2013, Thm 1.6].

We recall that O'Grady's original example is $\widetilde{M}_{(2,0,-2)}(S)$.

3.2. Pure dimension one sheaves and the manifolds $\widetilde{M}$ and $N$. By Theorem 3.1.2 (3), in order to study the Hodge numbers of $O G 10$-manifolds we are free to choose any Mukai vector $v=2 v^{\prime} \in H_{\text {alg }}^{\text {even }}(S, \mathbb{Z})$ with $v^{\prime 2}=2$. To make sure the corresponding projective model $\widetilde{M}_{2 v^{\prime}}(S)$ admits a Lagrangian fibration, we chose for $v^{\prime}$ the Mukai vector of a pure dimension 1 sheaves on $S$ [Ra-2008].

Recall that a coherent sheaf $\mathcal{F}$ on a scheme is said to be of pure dimension $d$ if the support of $\mathcal{F}$, as well as the support of all non-trivial subsheaves of $\mathcal{F}$, has dimension $d$ [Hu-Le-1997, §1.1]. Let $(S, H)$ be a polarized K3 surface with $N S(S)=\mathbb{Z} H$ and consider the positive Mukai vector:

$$
v=(0, k, \chi) .
$$

Pure sheaves on $S$ with Mukai vector $v$ are of pure dimension 1, have Euler characteristic $\chi$ and first Chern class $k H$. For example, if $\Gamma \in|k H|$ is a smooth curve and $i: \Gamma \rightarrow S$ is the closed embedding, then sheaves with Mukai vector equal to $v$ and support $\Gamma$ are of the form $i_{*} L$, where $L$ is a line bundle on $\Gamma$ with $\chi(L)=\chi$.

The Fitting support of a pure dimension one sheaf on $S$ is a pure dimension 1 subscheme on $S$ which represents the first Chern class of the sheaf and is defined as follows:

Remark 3.2.1. ( [LP-1993a, §2.2], [Hu-Le-1997, §1.1]) Let $\mathcal{F}$ be a pure dimension one sheaf on a smooth projective surface. Then $\mathcal{F}$ has depth one and hence admits a length one resolution

$$
0 \rightarrow A \stackrel{j}{\rightarrow} B \rightarrow \mathcal{F}
$$


where $A$ and $B$ are vector bundles on of the same rank $r$. The Fitting support Fitt $(\mathcal{F})$ is defined as the vanishing subscheme of the induced morphism $\operatorname{det} j: \operatorname{det} A \rightarrow \operatorname{det} B$. The scheme thus defined does not depend on the resolution, it always contains the set theoretic support of $F$, and it represents $c_{1}(\mathcal{F})$. For example, if $C^{\prime} \in S$ is a curve in the linear system $|H|, \mathcal{F}_{1}$ is the push forward to $S$ of a rank $k$ vector bundle on $C^{\prime}$ and $\mathcal{F}_{2}$ is the push forward to $S$ of a line bundle on $C:=k C^{\prime} \in|k H|$, then the Fitting support of $\mathcal{F}_{1}$ and $\mathcal{F}_{2}$ is the precisely the curve $C$.

Using the reduced Hilbert polynomial, we get the following definition of stability for pure dimension one sheaves.

Definition 3.2.2. (Gieseker stability for pure dimension one sheaves [Hu-Le-1997, Def. 1.2.3, Prop 1.2.6]) A pure dimension 1 sheaf $\mathcal{F}$ on a polarized surface $(X, H)$ is called Gieseker-(semi)stable with respect to $H$ if and only if for all proper pure dimension one quotients $\mathcal{F} \rightarrow \mathcal{E}$ the following inequality holds:

$$
\frac{\chi(\mathcal{F})}{c_{1}(\mathcal{F}) \cdot H} \underset{(=)}{<} \frac{\chi(\mathcal{E})}{c_{1}(\mathcal{E}) \cdot H} .
$$

For example, if the Fitting support of $\mathcal{F}$ is integral, then $\mathcal{F}$ is the push forward of a rank 1 torsion free sheaf on an integral curve, so $\mathcal{F}$ has no proper quotient and is automatically stable. If $\mathcal{F}$ is the pushforward of line bundle on a curve $C$, then the only pure dimension 1 proper quotients of $\mathcal{F}$ are the restrictions to the pure dimension one subschemes of $C$. It follows that $\mathcal{F}$ is (semi)-stable if and only if

$$
\frac{\chi(\mathcal{F})}{C \cdot H} \underset{(=)}{<} \frac{\chi\left(\mathcal{F}_{\mid D}\right)}{D \cdot H}
$$

for every proper subcurve $D \subset C$. In Lemmas 3.3.2 and 4.3.7 and Proposition 4.3.8 we will consider the stability of pure dimension one sheaves on certain non reduced curves.

Remark 3.2.3. (Non generic polarizations for pure dimension one sheaves) $A$ priori, the notion of stability depends on the choice of polarization. Since in this paper we are mostly concerned with the case of a Picard rank one surface, the polarization is unique up to scalars and we omit it from the notation for the moduli spaces and simply write $M_{v}(S)$. However, since in Theorem $B^{\prime}$ we consider moduli spaces of sheaves on K3 surfaces of higher Picard rank, we now recall some facts about this more general setting. Consider a positive $v$ (cf. Remark 3.1.1). If $v$ is primitive, then there is always a polarization for which there are no strictly semistable sheaves, i.e., all semistable sheaves are in fact stable; if $v=k v^{\prime}$ is not primitive, then there is always a polarization for which the summands of the polystable sheaves with Mukai vector $v$ have Mukai vector a multiple of $v^{\prime}$ [Hu-Le-1997, §4.C], [Yos-01]. For a given $v$, a polarization with these properties is called v-generic. If $v$ is primitive and $L$ is a v-generic polarization, then the moduli space $M_{v, L}(S)$ of L-stable sheaves on $S$ with Mukai vector $v$ is an irreducible holomorphic symplectic manifold of dimension $v^{2}+2$, deformation equivalent to the Hilbert scheme of $v^{2} / 2+1$ points on $S$. The birational class of $M_{v, L}(S)$ is independent of $L$ [Ba-Ma-2014a]. If $v=2 v^{\prime}$ with $\left(v^{\prime}\right)^{2}=2$ and $H$ is any polarization, then $M_{2 v^{\prime}, H}(S)$ has a symplectic resolution $\widetilde{M}_{2 v^{\prime}, H}(S)$, which is of OG10-type and whose birational class does not depend on H ([Le-So-2006], [Pe-Ra-2013, Thm 1.6], [Ar-Sa-2018, Prop 2.5],[Me-Zh-2016]). 
Let $g$ be the genus of the general curve in $|k H|$, so that $|k H| \cong \mathbb{P}^{g}$. The Le Potier morphism [LP-1993a, §2.2],[LP-1993b, §2.3]:

$$
\pi: M_{v}(S) \rightarrow \mathbb{P}^{g},
$$

associates with each sheaf its Fitting support. Since the fiber of $\pi$ over an integral curve is precisely the degree $d$ compactified Jacobian of the curve itself, by abuse of notation we refer to $M_{v}(S)$ as the relative compactified Jacobian of degree $d:=\chi+g-1$ of the universal curve over $|k H|$. Note, however, that if curve is non-reduced, the locus in a fiber parametrizing line bundles might be empty (cf. Corollary 4.3.9). By [Mu-1984, Bea-1991], the morphism (12) has Lagrangian fibers when restricted to the smooth locus $M_{v}(S)^{\text {reg }}$.

In order for $v=2 v^{\prime}$ with $\left(v^{\prime}\right)^{2}=2$ to be the Mukai vector of pure dimension one sheaves, one needs $v_{0}=0$ and $H^{2}=2$. To achieve this consider a polarized K3 surface of degree 2, i.e., a surface $S$ realized as a $2: 1$ cover:

$$
r: S \rightarrow \mathbb{P}^{2},
$$

ramified along a smooth plane sextic curve. The linear system $H:=r^{*} \mathcal{O}_{\mathbb{P}^{2}}(1)$ is of genus 2 , i.e., its general member is a nonsingular connected curve of genus 2 and $|H| \cong \mathbb{P}^{2}$. If the smooth sextic curve is general then $N S(S) \simeq \mathbb{Z}\langle H\rangle[\mathrm{Bu}]$. From now on, unless otherwise stated, we will assume that $S$ is a general in this sense. The Mukai vector

$$
v^{\prime}:=\left(0,1, \chi^{\prime}\right)
$$

is positive and satisfies $v^{\prime 2}=2$. With these choices, the moduli space $M_{v^{\prime}}(S)$ is a smooth projective irreducible holomorphic symplectic fourfold, with a Lagrangian fibration

$$
M_{v^{\prime}}(S) \rightarrow|H|=\mathbb{P}^{2},
$$

realizing it as the relative compactified Jacobian of degree $d^{\prime}:=\chi^{\prime}+1$ of the universal curve $\mathcal{C}^{\prime} /|H|$.

Remark 3.2.4. For general $S$ as above, the curves in $|H|$ are reduced and irreducible and have only nodes and (simple) cusps as singularities. Moreover, the rational curves in the linear systems have only nodes [Ch-2002]. By [Re-1980], the fibers of (14) are irreducible of dimension 2 .

The linear system $|2 H|$ is base point free and has dimension 5, and its general member is a connected nonsingular hyperelliptic curve of genus 5 [SD-1972], which is a ramified double cover of a plane conic. Consider the Mukai vector:

$$
2 v^{\prime}=\left(0,2,2 \chi^{\prime}\right) \text {. }
$$

The support morphism $M_{2 v^{\prime}}(S) \rightarrow|2 H|$ realizes this 10-dimensional singular moduli space as the relative compactified Jacobian of degree $d=2 \chi^{\prime}+4$ of the universal curve $\mathcal{C} /|2 H|$. By [Ma-2000, Thm 1], composing this morphism with the symplectic resolution gives the structure of Lagrangian fibration (cf. §2.3)

$$
\widetilde{M}_{2 v^{\prime}}(S) \rightarrow M_{2 v^{\prime}}(S) \rightarrow|2 H| \cong \mathbb{P}^{5},
$$

on this projective manifold $O G 10$-type. We also consider the moduli space associated with the primitive Mukai vector:

$$
w=(0,2, \chi), \quad \text { with } \chi \text { odd } .
$$


With this choice of Mukai vector, the moduli space $M_{w}(S)$ is a smooth irreducible holomorphic symplectic 10-fold of K3 $3^{[5]}$-type, with Lagrangian fibration $M_{w}(S) \rightarrow|2 H|$ realizing it as odd-degree $\chi+4$ relative compactified Jacobian of the universal curve $\mathcal{C} /|2 H|$.

Remark 3.2.5. (The subvarieties $\Delta \subseteq \Sigma \subseteq|2 H|$ ) The non integral curves in $|2 H| \simeq$ $\mathbb{P}^{5}$ are the preimages under $r$ of the non integral conics. They are parametrized by an irreducible divisor $\Sigma \subset|2 H|$, which is identified with $\mathrm{Sym}^{2}|H| \simeq \mathrm{Sym}^{2} \mathbb{P}^{2}$. The nonreduced curves are the preimages of the non-reduced conics (double lines) and they are parametrized by the irreducible 2-dimensional subvariety $\Delta_{|H|} \subset \operatorname{Sym}^{2}|H| \subset|2 H|$.

The analysis of the irreducible components of the fibers of $\widetilde{M}_{2 v^{\prime}}(S) \rightarrow|2 H|$ and $M_{w}(S) \rightarrow$ $|2 H|$ over $\Sigma$ and $\Delta$ plays a crucial role in this paper and will be carried out in Propositions 4.2.1, 4.2.2, and 4.2.4. For the moment, we review the following general properties.

Remark 3.2.6. (Flatness of the Lagrangian fibrations $\widetilde{M}_{2 v^{\prime}}(S), M_{w}(S), M_{v^{\prime}}(S)$, and $M_{2 v^{\prime}}(S)$.) The varieties $\widetilde{M}_{2 v^{\prime}}(S), M_{w}(S)$ and $M_{v^{\prime}}(S)$ are irreducible holomorphic symplectic manifolds. By [Ma-2000], the Lagrangian fibrations $\widetilde{M}_{2 v^{\prime}}(S) \rightarrow \mathbb{P}^{5}, M_{w}(S) \rightarrow \mathbb{P}^{5}$, and $M_{v^{\prime}}(S) \rightarrow \mathbb{P}^{2}$ are equidimensional and therefore flat. The following three facts concerning the morphism $M_{2 v^{\prime}}(S) \rightarrow|2 H| \simeq \mathbb{P}^{5}$ hold true: (i) since $M_{2 v^{\prime}}(S)$ has a symplectic resolution, it has canonical singularities, and is thus Cohen-Macaulay (see Theorem 5.10 and Corollary 5.24 of [Ko-Mo-1998]); (ii) since the fibers of $M_{2 v^{\prime}}(S) \rightarrow|2 H| \simeq \mathbb{P}^{5}$ are dominated by the corresponding fibers of $\widetilde{M}_{2 v^{\prime}}(S) \rightarrow \mathbb{P}^{5}$, they all have the same dimension five; the base of the fibration $M_{2 v^{\prime}}(S) \rightarrow|2 H|$ is nonsingular. By [Ma-1986, Thm 23.1], the morphism $M_{2 v^{\prime}}(S) \rightarrow|2 H|$ is flat. As a consequence, the fibers of $M_{2 v^{\prime}}(S) \rightarrow|2 H|$, $\widetilde{M}_{2 v^{\prime}}(S) \rightarrow \mathbb{P}^{5}, M_{w}(S) \rightarrow \mathbb{P}^{5}$, and $M_{v^{\prime}}(S) \rightarrow \mathbb{P}^{2}$ are Cohen-Macaulay.

For later use, we collect some known results:

Proposition 3.2.7. Let $v^{\prime}=\left(0,1, \chi^{\prime}\right)$ and let $v=(0,2, \chi)$. Denote by $S^{[n]}$ the Hilbert scheme of $n$ points of the K3 surface $S$.

(1) The birational class of the irreducible holomorphic symplectic manifold $M_{v^{\prime}}(S)$ depends on the parity of $\chi^{\prime}$ alone. If $\chi$ is odd, then $M_{v^{\prime}}(S)$ is an irreducible holomorphic symplectic manifold birational to $S^{[2]}$.

(2) The birational class of $M_{v}(S)$ depends on the parity of $\chi$ alone. If $\chi$ is odd, then $M_{v}(S)$ is an irreducible holomorphic symplectic manifold birational to $S^{[5]}$. If $\chi$ is even, then $M_{v}(S)$ is singular; blowing up its singular locus (with its reduced induced structure) produces an irreducible holomorphic symplectic manifold $\widetilde{M}_{v}(S)$ in the deformation class $O G 10$;

(3) For odd $\chi^{\prime}$ and $\chi$, there are isomorphisms of integral Hodge structures $H^{*}\left(M_{v^{\prime}}(S), \mathbb{Z}\right) \cong$ $H^{*}\left(S^{[2]}, \mathbb{Z}\right)$ and $H^{*}\left(M_{v}(S), \mathbb{Z}\right) \cong H^{*}\left(S^{[5]}, \mathbb{Z}\right)$.

(4) The isomorphism class of the integral Hodge structure of $\widetilde{M}_{v}(S)$ is the same for every even $\chi$.

Proof. The general curves in the linear systems $|H|$ and $|2 H|$ are smooth hyperelliptic, so tensoring a line bundle supported on a smooth curve with the unique $g_{2}^{1}$ on its support defines birational maps $M_{\left(0,1, \chi^{\prime}\right)}(S) \rightarrow M_{\left(0,1, \chi^{\prime}+2\right)}(S)$ and $M_{(0,2, \chi)}(S) \rightarrow M_{(0,1, \chi+2)}(S)$. 
The statement that $M_{v^{\prime}}(S)$ and $M_{v}(S)$, with $\chi^{\prime}=\chi=1$, are birational to the relevant Hilbert scheme is well-known (cf. [Bea-1999]). Finally, the statements about the isomorphisms of Hodge structures follow from [Hu-2003, Thm 2.5 Cor. 2.6].

3.3. The group schemes. Recall the definitions (13), (15) and (16) of the Mukai vectors $v^{\prime}, 2 v^{\prime}$, and $w$. As we shall see in $\S 3.4$, each of the moduli space $M_{v^{\prime}}(S), M_{w}(S)$ and $\widetilde{M}_{2 v^{\prime}}(S)$ is part of the data forming a $\delta$-regular weak abelian fibration. We now introduce the relevant group schemes as open subsets:

$$
\begin{gathered}
P^{\prime} \subseteq M_{(0,1,-1)}(S), \quad \text { for } M_{v^{\prime}}(S), \text { and } \\
P \subseteq M_{(0,2,-4)}(S), \quad \text { for } M_{w}(S) \text { and } \widetilde{M}_{2 v^{\prime}}(S) .
\end{gathered}
$$

Lemma 3.3.1. For every Mukai vector $v$ as in (13), (15), and (16), the locus $P_{v}(S) \subset$ $M_{v}(S)$ parametrizing stable sheaves that are push forwards of line bundles on their schematic supports is a non empty Zariski open subset.

Proof. An application of Nakayama's Lemma shows that a pure dimension 1 sheaf $\mathcal{F}$ on $S$ is a line bundle on its schematic support if and only if every non zero fiber of $\mathcal{F}$ has rank 1. The predicated openness then follows from the upper semicontinuity of the rank of the fibers of a coherent sheaf, while the non-emptiness is clear for the general fiber, where the curves are integral.

We remark that while this open set $P_{v}(S)$ intersects non trivially the fibers corresponding to an integral curve [Re-1980], it may not intersect some fibers of the Le Potier morphism over the non-reduced locus (cf. Corollary 4.3.9 ).

The following lemma describes the sheaves corresponding to closed point of $P_{v}(S)$ in the cases we are interested in, i.e. for $v=(0,2, \chi)$. More general statements along these lines appear in Section 4.3 (cf. Proposition 4.3.1, Lemma 4.3.7, and Proposition 4.3.8).

Lemma 3.3.2. Let $\mathcal{F}$ be a coherent sheaf on $S$ with $v(\mathcal{F})=(0,2, \chi)$. Assume that $\mathcal{F}$ is the push forward of a line bundle on a curve $C \in|2 H|$.

(1) If $C=2 C^{\prime}$ for a curve $C^{\prime} \in|H|$, then the Euler characteristic $\chi$ is even, $\mathcal{F}$ is stable, and the degree of the restriction of $\mathcal{F}$ to $C^{\prime}$ is $\operatorname{deg}\left(\mathcal{F}_{\mid C^{\prime}}\right)=(\chi / 2)+2$.

(2) (a) If $C=C_{1}+C_{2}$, for $C_{1} \neq C_{2} \in|H|$ and $\chi$ is even, then the sheaf $\mathcal{F}$ is stable if and only if $\operatorname{deg}\left(\mathcal{F}_{\mid C_{1}}\right)=\operatorname{deg}\left(\mathcal{F}_{\mid C_{2}}\right)=(\chi / 2)+2$.

(b) If $C=C_{1}+C_{2}$, for $C_{1} \neq C_{2} \in|H|$, and $\chi$ is odd, the sheaf $\mathcal{F}$ is stable if and only if $\operatorname{deg}\left(\mathcal{F}_{\mid C_{1}}\right)=2+(\chi+1) / 2$ and $\operatorname{deg}\left(\mathcal{F}_{\mid C_{2}}\right)=2+(\chi-1) / 2$ or viceversa.

Proof. Let $L$ be the line bundle on $C$ whose push forward on $S$ is $\mathcal{F}$. In case 1) let $i: C^{\prime} \rightarrow$ $C$ be the inclusion and let $\mathcal{I}$ be the sheaf of ideals of $C^{\prime}$ in $C$. Since $L$ is a line bundle on $C=2 C^{\prime}$, tensoring by $L$ the exact sequence of $\mathcal{O}_{C}$-modules $0 \rightarrow \mathcal{I} \rightarrow \mathcal{O}_{C} \rightarrow i_{*} \mathcal{O}_{C^{\prime}} \rightarrow 0$ gives the exact sequence

$$
0 \rightarrow \mathcal{I} \otimes i_{*} L_{\mid C^{\prime}} \rightarrow L \rightarrow i_{*} L_{\mid C^{\prime}} \rightarrow 0 .
$$

Note that since $\mathcal{I}^{2}=0, \mathcal{I}$ is an $\mathcal{O}_{C^{\prime}}$-module which is isomorphic to $\omega_{C^{\prime}}^{\vee} \cong \mathcal{O}_{C^{\prime}}\left(-C^{\prime}\right)$ and has degree -2 on $C^{\prime}$ (cf. (26)). It follows that $\chi=\chi(\mathcal{F})=\chi(L)=2 \chi\left(L_{\mid C^{\prime}}\right)-$ 
$2=2 \chi\left(\mathcal{F}_{\mid C^{\prime}}\right)-2$, hence $\chi$ is even and $\chi\left(\mathcal{F}_{\mid C^{\prime}}\right)=(\chi / 2)+1$. Formula (11) becomes $\chi(\mathcal{F})<2 \chi\left(\mathcal{F}_{\mid C^{\prime}}\right)$, so the sheaf $\mathcal{F}$ is stable and, since the arithmetic genus of $C^{\prime}$ is 2 , we conclude that $\operatorname{deg}\left(\mathcal{F}_{\mid C^{\prime}}\right)=\chi\left(\mathcal{F}_{\mid C^{\prime}}\right)+1=(\chi / 2)+2$.

In case 2), by formula (11), the sheaf $\mathcal{F}$ is stable if and only if $\chi=\chi(\mathcal{F})<2 \chi\left(\mathcal{F}_{\mid C_{i}}\right)$ for $i=1,2$. Using that the arithmetic genus of $C$ is 5 and the arithmetic genus of $C_{i}$ is 2 , the last inequality becomes $\operatorname{deg}(\mathcal{F})-4<2\left(\operatorname{deg}\left(\mathcal{F}_{\mid C_{i}}\right)-1\right)$ or equivalently $\operatorname{deg}\left(\mathcal{F}_{\mid C_{i}}\right)>$ $(\operatorname{deg}(\mathcal{F}) / 2)-1$. Since $\operatorname{deg}\left(\mathcal{F}_{\mid C_{1}}\right)+\operatorname{deg}\left(\mathcal{F}_{\mid C_{2}}\right)=\operatorname{deg}(\mathcal{F})=\chi-4$ item (2) follows.

\section{Corollary 3.3.3.}

For $v=(0,1,-1)$ (respectively, $v=(0,2,-4))$, the open set

$$
P_{v}(S) \subset M_{v}(S)
$$

can be identified with the relative degree-0 Picard scheme $\mathrm{Pic}_{\mathcal{C}^{\prime} /|H|}^{0}$ of the family $\mathcal{C}^{\prime} /|H|$ of curves of the linear system $|H|$ (respectively, the relative degree-0 Picard scheme $\mathrm{Pic}_{\mathrm{C} /|2 H|}^{0}$ of the family $\mathrm{C} /|2 \mathrm{H}|$ of curves of the linear system $|2 \mathrm{H}|$ ).

Proof. We prove the statement for $v=(0,2,-4)$, since the proof for $v=(0,1,-1)$ is analogous. Since $|2 H|$ is an ample linear system on a K3 surface, for every $C \in|2 H|$ the space $H^{0}\left(C, \mathcal{O}_{C}\right)$ has dimension 1 . This implies that the family $\mathcal{C} /|2 H|$ is cohomologically flat, i.e., in the language of [Al-Kl-1980], the structure sheaf $\mathcal{O}_{\mathcal{C}}$ of $\mathcal{C}$ is $|2 H|$-simple.

By [Al-Kl-1980, Cor. 7.6] the étale sheafification of the relative Picard functor of the family $\mathcal{C} /|2 H|$ is represented by an algebraic space and the same holds for the étale sheafification of the relative degree-0 Picard functor by [EGA IV(3), Cor. 15.6.5] or [Bo-Lu-Ra-1990, §8.4, Thm 4]. The representing object of the latter functor is, by definition, the nonsingular algebraic space $\operatorname{Pic}_{\mathcal{C} /|2 H|}^{0} \rightarrow|2 H|$. By [Bo-Lu-Ra-1990, §9.3, Cor. 13] closed points of $\operatorname{Pic}_{\mathcal{C} /|2 H|}^{0}$ correspond to line bundles on curves of $|2 C|$ having degree zero on every integral subcurve. By Lemma 3.3.2 the nonsingular variety $P:=P_{(0,2,-4)}(S)$ parametrizes the same set of line bundles seen as sheaves on $S$ and, since $P$ is an open subset of $M_{(0,2,-4)}(S)$, it corepresents the corresponding moduli functor by [Hu-Le-1997, Thm 4.3.4]. It follows that there exists a bijective morphism $\varphi: \operatorname{Pic}_{\mathcal{C} /|2 H|}^{0} \rightarrow P_{(0,2,-4)}(S)$; since the algebraic spaces $\operatorname{Pic}_{\mathcal{C} /|2 H|}^{0}$ and $P_{(0,2,-4)}(S)$ are smooth, the morphism $\varphi$ is an isomorphism and the algebraic space $\operatorname{Pic}_{\mathcal{C} /|2 H|}^{0}$ is identified with the scheme $P_{(0,2,-4)}(S)$.

To fix ideas, in the remainder of the paper we work with the the following varieties and morphisms, which have been introduced in the previous sections:

$$
\begin{aligned}
& m^{\prime}: M^{\prime}:=M_{(0,1,1)}(S) \longrightarrow B^{\prime}:=|H| \\
& \widetilde{m}: \widetilde{M}:=\widetilde{M}_{(0,2,2)} \stackrel{b}{\longrightarrow} M:=M_{(0,2,2)} \stackrel{m}{\longrightarrow} B:=|2 H| \\
& n: N:=M_{(0,2,1)} \longrightarrow B \\
& p: P:=\operatorname{Pic}_{\mathcal{C} /|2 H|}^{0} \longrightarrow B \\
& p^{\prime}: P^{\prime}:=\operatorname{Pic}_{\mathcal{C}^{\prime} /|H|}^{0} \longrightarrow|H| .
\end{aligned}
$$


Remark 3.3.4. Everything we say in the remainder of the paper about $\widetilde{M}, N$, and $M^{\prime}$ can be formulated also for any other moduli space with Mukai vector equal to $2 v^{\prime}, w$, and $v^{\prime}$ as in $(13,15,16)$, respectively.

3.4. The manifolds $\widetilde{M}, N, M^{\prime}$ and $M^{\prime(2)}$ as $\delta$-regular weak abelian fibrations. The goal of this section is to prove the forthcoming Proposition 3.4.6, to the effect that the triples listed there are $\delta$-regular weak abelian fibrations as in Definition 2.2.1. Recall the notation (19). We start by lifting the action of $P$ on $M$ to an action of $P$ on $\widetilde{M}$.

Lemma 3.4.1. (The action of the Picard group) Tensoring a sheaf by a line bundle with the same Fitting support induces:

(1) a group scheme structure on $P$ over $B$ with actions $a_{M}: P \times_{B} M \rightarrow M$ and $a_{N}: P \times_{B} N \rightarrow N$, on $M$ and $N$, over $B$,

(2) a group scheme structure on $P^{\prime}$ over $B^{\prime}$ with an action $a_{M^{\prime}}: P^{\prime} \times{ }_{B^{\prime}} M^{\prime} \rightarrow M^{\prime}$ on $M^{\prime}$ over $B^{\prime}$.

Proof. This is a general statement. Tensoring a pure dimension one sheaf whose schematic support is contained in a curve $C$ by an element of $\operatorname{Pic}^{0}(C)$, does not change the Euler characteristic of the sheaf itself, nor of its quotient sheaves. By Definition 3.2.2, (semi)stability is preserved by this operation. The fact that this set theoretic action is algebraic follows from the fact that the fiber product of the Picard scheme and the moduli space corepresents the appropriate product functor.

The following corollary follows immediately, since $b: \widetilde{M} \rightarrow M$ is the blow up of a locus which is left invariant by the action of the group scheme.

Corollary 3.4.2. The action $P \times{ }_{B} M \rightarrow M$ lifts to a, necessarily unique, action $P \times{ }_{B}$ $\widetilde{M} \rightarrow \widetilde{M}$.

At this stage it is clear that the requirements (a)-(d) in the Definition 2.2.1 of weak abelian fibration are met. The following two lemmata ensure that the remaining requirements (e) and (f) are met as well.

Lemma 3.4.3. Let the notation be as in Lemma 3.3.1. The open subsets

$$
P_{(0,2,2)} \subset \widetilde{M}, \quad \text { respectively } P_{(0,1,1)} \subset M^{\prime},
$$

surject onto $B$, respectively $B^{\prime}$, and are torsors under the group scheme $P$, respectively $P^{\prime}$. The image of the open subset $P_{(0,2,1)} \subset N$ in $B$ is $\Sigma \backslash \Delta$ and $P_{(0,2,1)}$ is a torsor under the restriction of $P$ to $\Sigma \backslash \Delta$. Moreover, there is no open set of $N$ which is a $P$-torsor.

Proof. The proof uses results proved in the forthcoming $\S 4.2-4.3$. Since the curves in $|H|$ are integral (cf. Remark 3.2.4), the surjectivity of $P_{(0,1,1)} \rightarrow B^{\prime}$ follows from Rego's result on the irreducibility of the moduli space of rank 1 torsion free sheaves on an integral locally planar curve [Re-1980]. The surjectivity of $P_{(0,2,2)} \rightarrow B$ follows from Proposition 4.2.2 and Proposition 4.3.10. It is clear that the action of $P$ (and of $P^{\prime}$ ) on the moduli spaces is free on the open invariant sets parametrizing sheaves that are line bundles on their scheme theoretic support. The assertion about the image of $P_{(0,2,1)} \rightarrow B$ follows 
from Proposition 4.2.2 and Corollary 4.3.9. The last assertion follows from Remark 4.3.19 and Corollary 4.3.11.

Lemma 3.4.4. The actions of $P$ on $\widetilde{M}, M$ and $N$, and of $P^{\prime}$ on $M^{\prime}$ and on $M^{\prime(2)}$ have affine stabilizers.

Proof. Recall that the action of the group scheme on the moduli spaces of sheaves is given by tensorization. We start by considering the following case. Let $F$ be a rank $r$ vector bundle on a smooth projective curve $C$ and suppose $F \cong F \otimes L$, for $L \in \operatorname{Pic}^{0}(C)$. Taking determinants, we see that $L^{r} \cong \mathcal{O}_{C}$ so $L$ belongs to a finite subgroup of $\operatorname{Pic}^{0}(C)$. Now suppose that $F$ is a pure (see Definition 3.2.2) sheaf on a curve $C=\sum C_{i}$, where $C_{i}$ are the irreducible, but possibly non-reduced, components of $C$. If $L \in \operatorname{Pic}^{0}(C)$ is such that $F \cong F \otimes L$, then we have that

$$
F_{\mid \widetilde{C}_{i}^{r e d}} / T o r \cong F_{\mid \widetilde{C}_{i}^{r e d}} / \text { Tor } \otimes L_{\mid \widetilde{C}_{i}^{\text {red }}}
$$

where $j_{i}: \widetilde{C}_{i}^{r e d} \rightarrow C_{i}^{r e d} \rightarrow C_{i}$ is the normalization of the reduced underlying curve and where $F_{\mid \widetilde{C}_{i}^{\text {red }}} /$ Tor is the pullback of $F$ to $\widetilde{C}_{i}^{\text {red }}$, modulo its torsion. Since $F_{\mid \widetilde{C}_{i}^{\text {red }}} /$ Tor is a vector bundle on a smooth curve, we can apply the previous observation to conclude that there is an integer $r_{i}$ such that $L_{\mid \widetilde{C}_{i}^{\text {red }}}^{r_{i}} \cong \mathcal{O}_{\widetilde{C}_{i}^{\text {red }}}$. Hence we conclude that, up to a finite quotient group, $L$ lies in the kernel of the natural morphism:

$$
\operatorname{Pic}^{0}(C) \stackrel{\prod j_{l}^{*}}{\longrightarrow} \prod \operatorname{Pic}^{0}\left(\widetilde{C}_{i}^{r e d}\right)
$$

which is precisely the affine part of $\operatorname{Pic}^{0}(C)$. From these observations it follows that the points of $M, N$, or $M^{\prime}$ corresponding to stable sheaves all have affine stabilizers. Hence, the points of $M^{\prime(2)}$ also also have affine stabilizers. The same reasoning, applied to a polystable sheaf $F=F_{1} \oplus F_{2}$, shows that the points corresponding to the singular locus of $M$ also have affine stabilizers. Indeed, the stabilizer of a point corresponding to a polystable sheaf is contained in the automorphism group of the polystable sheaf, which is a product of affine linear groups. By Corollary 3.4.2 the morphism $b: \widetilde{M} \rightarrow M$ is equivariant, so the stabilizer of a point $m \in \widetilde{M}$ is contained in the stabilizer of $b(m) \in M$. Thus if the points of $M$ have affine stabilizers, so do the points of $\widetilde{M}$.

Lemma 3.4.5. (Polarizability of Tate module) The $\overline{\mathbb{Q}}_{\ell}$-adic counterparts $T_{\text {et, }} \overline{\mathbb{Q}}_{\ell}(-)$ of the Tate module $T(-)$ associated with $P \rightarrow B$-the relative $\mathrm{Pic}^{0}$ for $\mathcal{C} \rightarrow B$-, and with $P^{\prime} \rightarrow B^{\prime}$-the relative $\mathrm{Pic}^{0}$ for $\mathcal{C}^{\prime} \rightarrow B^{\prime}$-are polarizable. The same is true if we restrict the families of curves to any subfamily of curves over a locally closed subvariety of $B$, or of $B^{\prime}$.

Proof. The polarizability result [de-2017, Theorem 3.3.1], which is stated for the family of spectral curves for the $G L_{n}$-Hitchin system, is in fact proved for any family of curves obtained via base change from a linear system of curves on a nonsingular surface. Therefore, the polarizability result holds for the families of curves $\mathcal{C}^{\prime} \rightarrow B^{\prime}$ and $\mathcal{C} \rightarrow B$.

By putting together the preceding contents of this section, we get the following 
Proposition 3.4.6. ( $\delta$-regularity) The triples:

$$
(\widetilde{M}, B, P),(N, B, P),\left(M^{\prime}, B, P^{\prime}\right),\left(M^{\prime 2}, B^{\prime 2}, P^{\prime 2}\right),\left(\operatorname{Sym}^{2} M^{\prime}, \operatorname{Sym}^{2} B^{\prime}, \operatorname{Sym}^{2} P^{\prime}\right)
$$

are $\delta$-regular weak abelian fibrations satisfying the assumptions of Theorem 7.0.3 (Direct image is sum of Ngô strings). The group schemes in the triples appear in, or are related to, (58).

Proof. In view of Lemma 3.4.1, Corollary 3.4.2, and Lemma 3.4.4, all the triples above are weak abelian fibrations and we only need to prove $\delta$-regularity. In the cases $(\widetilde{M}, B, P)$ and $\left(M^{\prime}, B, P^{\prime}\right), \delta$-regularity follows from Proposition 2.3.2 and Lemma 3.4.3. In the cases $\left(M^{\prime 2}, B^{\prime 2}, P^{\prime 2}\right)$ and $\left(\mathrm{Sym}^{2} M^{\prime}, \mathrm{Sym}^{2} B^{\prime}, \mathrm{Sym}^{2} P^{\prime}\right), \delta$-regularity follow formally from the $\delta$ regularity of $\left(M^{\prime}, B, P^{\prime}\right)$. In the case $(N, B, P)$ it is not true that $N$ contains a $P$-torsor over $B$, so that this case is not covered directly by Proposition 2.3.2. On the other hand, $\delta$-regularity is a property of the group scheme $P$, and this has already been established when dealing with $(\widetilde{M}, B, P)$.

4. The top Direct image sheaf $R^{10}$ For the Lagrangian fibrations $\widetilde{M}, N \rightarrow B$

The comparison of the derived direct images of the constant sheaves of the two fibrations $\widetilde{M}, N \rightarrow B$ involves understanding the direct summands of these derived direct images, as well as their supports. Thanks to Ngô's Support Theorem, it is often enough to understand the top degree (in this case, degree 10) direct image sheaves. The aim of this section is to prove Proposition 4.4.3, which describes, over certain relevant subvarieties of $B$, the top direct image sheaves of the constant sheaf for the two fibrations. This result will be used in $\S 5$ to determine the Decomposition Theorem for the two fibrations. Note that as a simple consequence of the structure of the symplectic resolution $\widetilde{M} \rightarrow M$ (Fact 4.1.1), Corollary 4.1.4 immediately singles out three subvarieties of $B$ as supports for the Lagrangian fibration $\widetilde{M} \rightarrow B$. However, to show that these subvarieties are the only supports of the fibrations and to determine the summands on each support actually requires Proposition 4.4.3. A first step towards this proposition is to identify the irreducible components (as well as their monodromy) of the fibers of the equidimensional fibrations $\widetilde{M}, N \rightarrow B$. This identification is done in $\S 4.2$ and uses the results developed in $\S 4.3$.

4.1. The Decomposition Theorem for the blow up $b: \widetilde{M} \rightarrow M$.

By Theorem 3.1.2 and Remark 3.2.5, we have the following commutative diagram

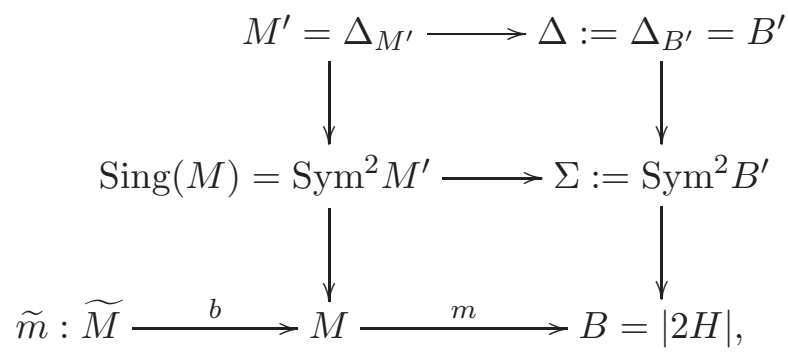


where the morphisms $\widetilde{m}$ and $m$ are as in (19) and the morphism $b$ is the blow up of $M$ along its singular locus $\operatorname{Sing}(M) \simeq \operatorname{Sym}^{2} M^{\prime}$ (with the reduced induced structure). The study of the local analytic structure of the singularities of $M$ from [Le-So-2006] gives the following:

Fact 4.1.1. (The symplectic resolution $b: \widetilde{M} \rightarrow M$ )[Le-So-2006, §4]. The morphism $b$ is semismall with irreducible exceptional divisor $E \subset \widetilde{M}$. The fibers of $E \rightarrow \operatorname{Sing}(M)$ are as follows:

(a) the morphism $E_{\mathrm{Sym}^{2}\left(M^{\prime}\right) \backslash \Delta_{M^{\prime}}} \rightarrow \operatorname{Sym}^{2}\left(M^{\prime}\right) \backslash \Delta_{M^{\prime}}$ is an analytic fiber bundle with fiber $\mathbb{P}^{1}$;

(b) the morphism $E_{\Delta_{M^{\prime}}} \rightarrow \Delta_{M^{\prime}}$ is an analytic fiber bundle with fiber a three dimensional smooth quadric.

For later use, we highlight the following consequence of diagram (20).

Remark 4.1.2. A point $b \in \Sigma \backslash \Delta$ corresponds to two distinct point $b_{1}, b_{2} \in B^{\prime}$; the strictly semistable locus $\operatorname{Sing}(M)_{b}=\left(\operatorname{Sym}^{2} M^{\prime}\right)_{b}$ of the fiber $M_{b}:=m^{-1}(b)$ is isomorphic to the product $M_{b_{1}}^{\prime} \times M_{b_{2}}^{\prime}$ of the corresponding fibers of $m^{\prime}: M^{\prime} \rightarrow B^{\prime}$. In particular, $\operatorname{Sing}(M)_{b}$ is irreducible of dimension 4.

Analogously, for $b \in \Delta$ the strictly semistable locus $\operatorname{Sing}(M)_{b}$ of the fiber $M_{b}$ is isomorphic to $\operatorname{Sym}^{2}\left(M^{\prime}\right)_{b}$ (recall the identification $\left.\Delta \simeq B^{\prime}\right)$. It follows that $\operatorname{Sing}(M)_{b}$ is irreducible of dimension 4 . The strictly semistable locus of $M_{\Delta}:=m^{-1}(\Delta)$ is the quotient of the fiber product $M^{\prime} \times_{\Delta} M^{\prime}$ by the involution exchanging the factors. For $b \in \Delta$, the intersection $M_{b} \cap \Delta_{M^{\prime}}$ has a unique irreducible component isomorphic to $M_{b}^{\prime}$, which is irreducible of dimension 2.

We can now determine the Decomposition Theorem for $\widetilde{M} \rightarrow M$.

Lemma 4.1.3. (Decomposition Theorem for the blow up $b: \widetilde{M} \rightarrow M$ ) There is a canonical isomorphism in $D^{b} M H M_{\text {alg }}(M)$ and in $D^{b}(M, \mathbb{Q})$ (turn off the Tate shifts):

$$
R b_{*} \mathbb{Q}_{\widetilde{M}} \simeq \mathscr{I} \mathscr{C}_{M} \bigoplus \mathbb{Q}_{\mathrm{Sym}^{2} M^{\prime}}[-2](-1) \bigoplus \mathbb{Q}_{\Delta_{M^{\prime}}}[-6](-3),
$$

Proof. By combining Fact 4.1.1 with the Decomposition Theorem for semismall morphisms (cf. the survey [de-Mi-2009], and references therein), we obtain a canonical isomorphism:

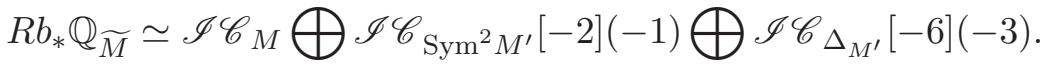

Since $M^{\prime}$ is nonsingular, the intersection complexes appearing as the second and third summand are actually the constant sheaf with the corresponding dimensional shift.

Corollary 4.1.4. There is a canonical isomorphism in $D^{b} M H M_{a l g}(S)$ and in $D^{b}(S, \mathbb{Q})$ :

$$
R \widetilde{m}_{*} \mathbb{Q}_{\widetilde{M}} \simeq R m_{*} \mathscr{I} \mathscr{C}_{M} \bigoplus R m_{*} \mathbb{Q}_{S^{\prime} m^{2} M^{\prime}}[-2](-1) \bigoplus R m_{*} \mathbb{Q}_{\Delta_{M^{\prime}}}[-6](-3) .
$$

In particular, the subvarieties $B, \Sigma, \Delta$ of $B$ are among the supports of $R \widetilde{m}_{*} \mathbb{Q}_{\widetilde{M}}$ on $B$.

Proof. Apply $R m_{*}$ to (22) and obtain (23). Each direct summand in (23) is the direct image of the intersection complex of a variety that surjects properly onto $B, \Sigma$ and $\Delta$, respectively; the statement about the supports follows from this. 
Remark 4.1.5. The forthcoming Proposition 5.7.2 shows that $B, \Sigma$ and $\Delta$ are the only supports of $R \widetilde{m}_{*} \mathbb{Q}_{\widetilde{M}}$ on $B$.

4.2. Irreducible components of the fibers: main results. In view of proving Proposition 4.4.3, which is concerned with the top degree direct image sheaves $R^{10} \widetilde{m}_{*} \mathbb{Q}_{\widetilde{M}}$ and $R^{10} n_{*} \mathbb{Q}_{N}$, in this subsection we study the irreducible components of the fibers of the fibrations $M, N \rightarrow B$ over the loci $\Sigma=\operatorname{Sym}^{2}|H|$ and $\Delta=\Delta_{|H|}$ of $B=|2 H|$.

The main results are: Proposition 4.2.1, dealing with the components of the fibers of $\widetilde{M}$ that arise from the exceptional divisor $E$; Proposition 4.2.2, on the structure of the fibers of $M$ and $N$ over the locus $B \backslash \Delta$ of reduced curves; and finally Proposition 4.2.4, on the structure of the fibers of $M$ and $N$ over the locus $\Delta$ of non-reduced curves. The proof of this last proposition rests on Propositions 4.3.10 and 4.3.16, which are the main results of the forthcoming $\S 4.3 .3$.

4.2.1. Irreducible components arising from the exceptional divisor. Let $\widetilde{m}_{E}: E \rightarrow \operatorname{Sing}(M)=$ Sym $^{2} M^{\prime} \rightarrow B$ be the restriction of $\widetilde{m}$ to the exceptional divisor of the symplectic resolution $b: \widetilde{M} \rightarrow M$. Set

$$
E_{\Delta}:=\widetilde{m}_{E}^{-1}(\Delta), \quad E_{\Delta}^{I}:=b^{-1}\left(\Delta_{M}^{\prime}\right), \quad E_{\Delta}^{I I}:=E_{\Delta} \backslash E_{\Delta}^{I} .
$$

Proposition 4.2.1 (The structure of the exceptional divisor over $\Delta$ ).

(1) The variety $E_{\Delta}$ is the union of two projective irreducible varieties of pure dimension 7 , namely $E_{\Delta}^{I}$ and the closure $\overline{E_{\Delta}^{I I}}$ of $E_{\Delta}^{I I}$ in $E_{\Delta}$;

(2) The morphism $E_{\Delta}^{I} \rightarrow \Delta$ is projective, with irreducible 5-dimensional fibers;

(3) The morphism $E_{\Delta}^{I I} \rightarrow \Delta$ is flat, surjective, with irreducible 5-dimensional fibers.

Proof. Part (1) follows from parts (2) and (3), which we now prove. (2) By Fact 4.1.1 (b), the morphism $E_{\Delta}^{I} \rightarrow \Delta$ factors via a 3-dimensional quadric fibration $E_{\Delta}^{I} \rightarrow \Delta_{M^{\prime}}=M^{\prime}$, followed by the relative compactified Jacobian $m^{\prime}: M^{\prime} \rightarrow \Delta=|H|$ of the genus 2 linear system $|H|$. As noted in Remark 3.2.4, the curves in $|H|$ are reduced and irreducible and hence so are the fibers of $m^{\prime}$ [Re-1980]. (3) By definition of $E_{\Delta}^{I I}$, there is a factorization

$$
E_{\Delta}^{I I} \rightarrow \operatorname{Sym}_{\Delta}^{2}\left(M^{\prime}\right) \backslash \Delta\left(M^{\prime}\right) \rightarrow \Delta
$$

where $\operatorname{Sym}_{\Delta}^{2}\left(M^{\prime}\right)=\left(\operatorname{Sym}^{2}\left(M^{\prime}\right)\right)_{\Delta}$ is the quotient of the fiber product $M^{\prime} \times_{\Delta} M^{\prime}$ by the involution interchaging the two factors. By Fact 4.1.1 (a), $E_{\Delta}^{I I} \rightarrow \operatorname{Sym}_{\Delta}^{2}\left(M^{\prime}\right) \backslash \Delta\left(M^{\prime}\right)$ is a fibration in $\mathbb{P}^{1}$ and, by Remark 3.2.6, $\operatorname{Sym}_{\Delta}^{2}\left(M^{\prime}\right) \backslash \Delta\left(M^{\prime}\right) \rightarrow \Delta$ is flat surjective with irreducible 4-dimensional fibers.

4.2.2. Irreducible components over reduced curves.

For $b \in B$ representing a reduced curve, a description of the fibers $M_{b}:=m^{-1}(b)$, $N_{b}:=n^{-1}(b)$ and $\widetilde{M}_{b}:=\widetilde{m}^{-1}(b)$ can be found in [Ra-2008]. In the following proposition and corollary we collect the result that we will use in this paper.

Proposition 4.2.2. Consider a point $b \in B \backslash \Delta$. The fibers $M_{b}$ and $N_{b}$ are reduced, Cohen-Macauley of dimension 5 and have dense open subsets parametrizing line bundles. The fiber $M_{b}$ is irreducible while the fiber $N_{b}$ is irreducible for $b \in B \backslash \Sigma$ and has exactly 2 irreducible components for $b \in \Sigma \backslash \Delta$. 
Proof. By Remark 3.2.6, the morphisms $m$ and $n$ are flat and the fibers are Cohen-Macauly of dimension 5. For $b \in B \backslash \Delta$, the points of the fibers $M_{b}$ and $N_{b}$ represent rank 1 torsion free sheaves on the reduced locally planar curve $C_{b}$ corresponding to $b$. By Proposition 2.3 (ii) of [Me-Ra-Vi-2017], the loci of $M_{b}$ and $N_{b}$ representing stable line bundles are dense in $N_{b}$ and in the stable locus of $M_{b}$, respectively. Since the strictly semistable locus of $M_{b}$ has dimension 4 (see Remark 4.1.2), and since $M_{b}$ is 5 dimensional and Cohen-Macauley, density of the line bundle locus holds also in $M_{b}$. By Proposition 2.8 of [LP-1993a] the Le Potier morphism is smooth at every point corresponding to a stable line bundle: hence $M_{b}$ and $N_{b}$ have open dense subsets which are reduced. Since $M_{b}$ and $N_{b}$ are Cohen-Macauley, they are reduced everywhere.

Finally, using Corollary 13 of Chapter 9 of [Bo-Lu-Ra-1990], every connected component $\Gamma$ of the loci of $M_{b}$ or $N_{b}$ parametrizing stable line bundles is determined by the set of degrees of the restrictions of any line bundle parametrized by $\Gamma$ to the irreducible components of $C_{b}$. If $b \in B \backslash \Sigma$, then the corresponding curve $C_{b}$ as well as the fibers $M_{b}$ and $N_{b}$ are irreducible [Re-1980]. If $b \in \Sigma \backslash \Delta$, the curve $C_{b}$ has 2 distinct irreducible components so by Lemma 3.3.2(2) the fiber $M_{b}$ is irreducible while the fiber $N_{b}$ has two irreducible components.

Corollary 4.2.3. For $b \in \Sigma \backslash \Delta$, the fiber $\widetilde{M}_{b}$ is pure of dimension 5 and has exactly 2 irreducible components.

Proof. By Proposition 4.2.2 the strict transform of $M_{b}$ in $\widetilde{M}_{b}$ is irreducible of dimension 5 . By Fact 4.1.1 and Remark 4.1.2 the inverse image of the strictly semistable locus $\operatorname{Sing}(M)_{b}$ of $\widetilde{M}_{b}$ is a $\mathbb{P}^{1}$-bundle over an irreducible variety of dimension 4 .

4.2.3. Irreducible components over non-reduced curves: the statement. Next, we analyze the irreducible components of the fibers of $M, N \rightarrow B$ over the points $b \in \Delta \subseteq B$ parametrizing non-reduced curves. This analysis divides the sheaves parametrized by the fibers over $\Delta$ into two types, which we call type I (sheaves defined on the reduction of the curves) and type II (the remaining ones). Consider the restrictions

$$
N_{\Delta} \rightarrow \Delta, \quad M_{\Delta} \rightarrow \Delta
$$

of the morphisms $N, M \rightarrow B$ to the locus of double curves $\Delta \subseteq B$. The main result of this subsection is Proposition 4.2.4, which describes the irreducible components of the varieties $N_{\Delta}$ and $M_{\Delta}$, as well as of their fibers $N_{C}$ and $M_{C}$ over the points $C \in \Delta$. The proof of this proposition uses results from $\S 4.3$ on certain sheaves on non-reduced curves.

Recall from Remark 3.2.5 that every curve $C \in \Delta \subseteq|2 H|$ is of the form $C=2 C^{\prime}$ for a curve $C^{\prime} \in|H|$. Let us introduce the sheaves of type $I$ and $I I$. The sheaves parametrized by $N_{\Delta}$ and $M_{\Delta}$ are of pure dimension 1 on $S$ and their Fitting supports are non-reduced curves $C=2 C^{\prime} \in \Delta$. There are two kinds of such sheaves. The first kind are the sheaves $\mathcal{F}$ on $S$ for which the compositum of the natural morphisms $\mathcal{O}_{S} \rightarrow \mathcal{O}_{C} \rightarrow \mathcal{E} n d_{S}(\mathcal{F})$ factors via the natural surjection $\mathcal{O}_{C} \rightarrow \mathcal{O}_{C^{\prime}}$. We call these the sheaves of type $I$; in this case, $\mathcal{F}$ is an $\mathcal{O}_{C^{\prime}}$-module and can be viewed as a rank 2 torsion free sheaf on the underlying reduced curve $C^{\prime}$; we denote the corresponding loci by $M_{C}^{I}$ and $N_{C}^{I}$ and, by letting $C$ vary 
in $\Delta, M_{\Delta}^{I}$ and $N_{\Delta}^{I}$. We call the remaining sheaves of type $I I$, and we set

$$
M_{C}^{I I}:=M_{C} \backslash M_{C}^{I}, \quad, N_{C}^{I I}:=N_{C} \backslash N_{C}^{I}, \quad M_{C}^{I I}:=M_{\Delta} \backslash M_{\Delta}^{I}, \quad N_{C}^{I I}:=N_{\Delta} \backslash M_{\Delta}^{I} .
$$

In other words, the sheaves of type $I I$ on $C=2 C^{\prime}$ are those for which the Fitting support equals the schematic support. In the following proposition, we consider the varieties and fibers with their reduced induced structure.

Proposition 4.2.4. (Irreducible components of $M_{\Delta}, N_{\Delta} \rightarrow \Delta$ )

(1) (a) The variety $M_{\Delta}$ is of pure dimension 7 and has two irreducible components, namely $M_{\Delta}^{I}$ and the closure $\overline{M_{\Delta}^{I I}}$ of $M_{\Delta}^{I I}$.

(b) The morphisms $M_{\Delta}^{I}, M_{\Delta}^{I I} \rightarrow \Delta$ have irreducible 5-dimensional fibers, namely, for $C \in \Delta, M_{C}^{I}$ and $M_{C}^{I I}$.

(2) (a) The variety $N_{\Delta}$ is of pure dimension 7 and has two irreducible components, namely $N_{\Delta}^{I}$ and the closure $\overline{N_{\Delta}^{I I}}$ of $N_{\Delta}^{I I}$.

(b) The morphisms $N_{\Delta}^{I}, N_{\Delta}^{I I} \rightarrow \Delta$ have irreducible 5-dimensional fibers, namely, for $C \in \Delta, N_{C}^{I}$ and $N_{C}^{I I}$.

Proof. The proof uses results from the forthcoming $\S 4.3$ : by Proposition 4.3.1 the varieties $M_{C}^{I}$ and $N_{C}^{I}$ are irreducible of dimension 5 and $M_{\Delta}^{I}$ and $N_{\Delta}^{I}$ are irreducible of dimension 7. By Proposition 4.3.10 the variety $M_{C}^{I I}=M_{C} \backslash M_{C}^{I}$ is irreducible of dimension 5 and, by Proposition 4.3.16, the same holds for $N_{C}^{I I}=N_{C} \backslash N_{C}^{I}$. By Remark 3.2.6, the varieties $M_{\Delta}$ and $N_{\Delta}$ are flat over $\Delta$. Since the fibers of $M_{\Delta}^{I I}=M_{\Delta} \backslash M_{\Delta}^{I}$ and $N_{\Delta}^{I I}=N_{\Delta} \backslash N_{\Delta}^{I}$ are irreducible of dimension 5 , the closure $\overline{M_{\Delta}^{I I}}$ of $M_{\Delta}^{I I}$ in $M_{\Delta}^{I I}$ and the closure $\overline{N_{\Delta}^{I I}}$ of $N_{\Delta}^{I I}$ in $N_{\Delta}^{I I}$ are irreducible of dimension 7.

Remark 4.2.5. The varieties $M_{\Delta}^{I I}, M_{C}^{I I}$ (and their respective closures) are actually reduced but we don't need this result.

4.3. The fibers over non-reduced curves. In this section we study sheaves the nonreduced curves of $|2 H|$ with the aim of proving Propositions 4.3.1, 4.3.10, and 4.3.16, which were used in the proof of Proposition 4.2.4. First we deal with the simpler case of the sheaves of type I (§4.3.1), then in $\S 4.3 .2-4.3 .3$ we carry out the more involved study of the sheaves of type II.

\subsubsection{Irreducible components over non-reduced curves: sheaves of type I.}

Proposition 4.3.1. (Irreducibility of $M_{C}^{I}, N_{C}^{I}, M_{\Delta}^{I}$ and $N_{\Delta}^{I}$ ) Let $C=2 C^{\prime} \in \Delta$.

(1) The locus $M_{\Delta}^{I}$ is an irreducible component of $M_{\Delta}$ and the morphism $M_{\Delta}^{I} \rightarrow \Delta$ is projective with irreducible 5-dimensional fibers $M_{C}^{I}$.

(2) The locus $N_{\Delta}^{I}$ is an irreducible component of $N_{\Delta}$ and the morphism $N_{\Delta}^{I} \rightarrow \Delta$ is projective with irreducible 5-dimensional fibers $N_{C}^{I}$.

Proof. The sheaves of type $I$ are $\mathcal{O}_{C^{\prime}}$-modules, so by Definition 3.2.2 the loci $M_{C}^{I}$ and $N_{C}^{I}$ parametrizing semistable sheaves of type $I$ on $C$, with Euler characteristic $\chi$, are naturally identified with the moduli spaces of torsion free slope semistable sheaves on the reduced underlying curve $C^{\prime}$, with rank 2 and degree $d^{\prime}:=\chi-2 \chi\left(\mathcal{O}_{C^{\prime}}\right)$. The latter space 
is irreducible and projective by [Se-1982, Re-1982]. By [Hu-Le-1997, Thm 4.3.7], $M_{\Delta}^{I}$ and $N_{\Delta}^{I}$ are projective over $\Delta$. The statement about the dimension of the fibers follows from Remark 3.2.6. This proves the first assertion. The second one follows, since the morphisms $M_{\Delta}^{I}, N_{\Delta}^{I} \rightarrow \Delta$ are proper, surjective and with irreducible fibers.

As a side remark, we mention that it is a classical result of [Na-Ra-1969], that for a smooth curve $C^{\prime} \in B^{\prime}$, the assignment $\mathcal{F} \mapsto \operatorname{det} \mathcal{F}$ realizes $N_{C}^{I}$ (respectively, $M_{C}^{I}$ ) as a smooth fibration over $\mathrm{Jac}^{d^{\prime}}\left(C^{\prime}\right)$, locally trivial in the étale topology, with fibers a transversal complete intersections of two 4-dimensional quadrics (respectively, $\mathbb{P}^{3}$ ).

Remark 4.3.2. The strictly semistable locus on $N$ is empty. By Theorem 3.1.2 (1) the locus of strictly semistable sheaves in $M_{\Delta}$ is contained in $M_{\Delta}^{I}$.

4.3.2. Preliminary results towards the analysis of type II.

In this subsection we establish some notation, recall some facts, and prove a series of preliminary results towards Propositions 4.3.10 and 4.3.16 (which describes the fibers of $\left.M_{\Delta}^{I I}, N_{\Delta}^{I I} \rightarrow \Delta\right)$.

For $C^{\prime} \in B^{\prime}$, let $C=2 C^{\prime} \in \Delta$ be the corresponding double curve. Denote by $\mathcal{I} \subset \mathcal{O}_{C}$ the ideal sheaf of the reduced curve $C^{\prime} \subset C$. Then:

$$
\mathcal{I}^{2}=0, \quad \mathcal{I} \text { is an } \mathcal{O}_{C^{\prime}} \text {-module, } \mathcal{I} \cong \mathcal{O}_{C^{\prime}}\left(-C^{\prime}\right) \cong \omega_{C^{\prime}}^{-1}, \operatorname{deg} \mathcal{I}=-2 .
$$

Since the genus 2 K3 surface $S$ is general in moduli, any curve $C^{\prime} \in|H|$ has only nodes and cusps (cf. Remark 3.2.4) and, moreover, it has at most two singular points. For later use we record the following standard facts on rank 1 torsion free sheaves on integral curves with nodes or cusps. Let $\Gamma$ be an integral projective curve with only nodes and cusps and let $E$ be a rank one torsion free sheaf on $\Gamma$. By [DS-1979, Lem 1.4], we can write $E=n_{*} L$, where $n: \widehat{\Gamma} \rightarrow \Gamma$ is a partial normalization and where $L$ is a line bundle on $\widehat{\Gamma}$. Both $\widehat{\Gamma}$ and $L$ are uniquely determined by $E$. Now let $\mathcal{L} \in \operatorname{Pic}(\Gamma)$ be a line bundle. Then $E \otimes \mathcal{L} \cong E$ if and only if $\mathcal{L} \in \operatorname{Ker}\left[n^{*}: \operatorname{Pic}(\Gamma) \rightarrow \operatorname{Pic}(\widehat{\Gamma})\right]$ (cf. [Bea-1999, Lem. 2.1]).

Lemma 4.3.3. (Nodes and cusps) Let $\Gamma$ be as above.

(1) Let $q \in \Gamma$ be any point. Then $\operatorname{Ext}_{\mathcal{O}_{\Gamma}}^{1}\left(\mathbb{C}_{q}, \mathcal{O}_{\Gamma}\right)=\operatorname{Ext}_{\mathcal{O}_{\Gamma, q}}^{1}\left(\mathbb{C}_{q}, \mathcal{O}_{\Gamma, q}\right)=\mathbb{C}$. The unique (up to isomorphism) extension is:

$$
0 \rightarrow \mathcal{O}_{\Gamma} \rightarrow\left(m_{q}\right)^{\vee} \rightarrow \mathbb{C}_{q} \rightarrow 0,
$$

obtained by dualizing the standard exact sequence $0 \rightarrow m_{q} \rightarrow \mathcal{O}_{\Gamma} \rightarrow \mathbb{C}_{q} \rightarrow 0$.

(2) Let $q \in \Gamma$ be a singular point, and let $\widehat{\Gamma}$ be the normalization of $\Gamma$ at $q$. Then $\operatorname{Ext}_{\mathcal{O}_{\Gamma}}^{1}\left(\mathbb{C}_{q}, m_{q}\right)=\operatorname{Ext}_{\mathcal{O}_{\Gamma, q}}^{1}\left(\mathbb{C}_{q}, m_{q}\right)=2$. The corresponding 1 -dimensional family of isomorphism classes of extensions is given by considering the natural extension

$$
0 \rightarrow m_{q} \rightarrow \mathcal{O}_{\Gamma} \rightarrow \mathbb{C}_{q} \rightarrow 0
$$

and tensoring it with the elements of the one dimensional group $\operatorname{Ker}\left[n^{*}: \operatorname{Pic}(\Gamma) \rightarrow\right.$ $\operatorname{Pic}(\widehat{\Gamma})]$ (which fixes $\left.m_{q}\right)$.

Proof. (1) Since $\Gamma$ is a Gorenstein curve, have $\operatorname{dim} \operatorname{Ext}_{\mathcal{O}_{\Gamma, q}}^{1}\left(\mathbb{C}_{q}, \mathcal{O}_{\Gamma, q}\right)=1$. (2) The first statement follows from [Co-1993, Lem. 2.5.5]. The second statement follows from the considerations before the Lemma, with $E=m_{q}$ and $\widehat{\Gamma}$ the normalization of $\Gamma$ at $q$. 
Lemma 4.3.4. ([Co-1993, Lem. 2.5.9]) Let $\Gamma$ be an integral curve, let $p \in \Gamma$ be a nodal or cuspidal point, and let $F_{p}$ and $K_{p}$ be rank 1 torsion free $\mathcal{O}_{\Gamma, p}$-modules. Then

$$
\operatorname{Ext}_{\Gamma, p}^{1}\left(F_{p}, K_{p}\right)=\left\{\begin{array}{cc}
0 & \text { if } F_{p}, \text { or } K_{p} \text { is free, } \\
\mathbb{C}^{2} & \text { if neither } F_{p}, \text { nor } K_{p} \text { is free. }
\end{array}\right.
$$

Lemma 4.3.5. Let $n: \widehat{\Gamma} \rightarrow \Gamma$ be a partial normalization of a reduced curve and let $L$ and $L^{\prime}$ be torsion free sheaves on $\widehat{\Gamma}$. Then $\operatorname{Hom}_{\Gamma}\left(n_{*} L, n_{*} L^{\prime}\right)=\operatorname{Hom}_{\widehat{\Gamma}}\left(L, L^{\prime}\right)$.

Proof. It is clear that a morphism $\varphi: L \rightarrow L^{\prime}$ induces by pushforward a morphism $n_{*} \varphi: n_{*} L \rightarrow n_{*} L^{\prime}$ of torsion free sheaves. Conversely, if $\psi: n_{*} L \rightarrow n_{*} L^{\prime}$ is a morphism, by pullback we get a morphism $n^{*} n_{*} L \rightarrow n^{*} n_{*} L^{\prime} \rightarrow L^{\prime}$. Since $L^{\prime}$ is torsion free, this morphism necessarily factors via the surjection $n^{*} n_{*} L \rightarrow L$ which thus induces a morphism $L \rightarrow L^{\prime}$. Since $n$ is a generic isomorphism, these two assignments are inverses of each other.

Lemma 4.3.6. Let $E$ be a pure dimension 1 sheaf on the $K 3$ surface $S$ and let $\Gamma \subseteq S$ be the curve Fitting support of $E$. Then $\mathcal{E x t}_{S}^{i}\left(E, \mathcal{O}_{S}(-\Gamma)\right)=0$ for $i \neq 1$ and there is a

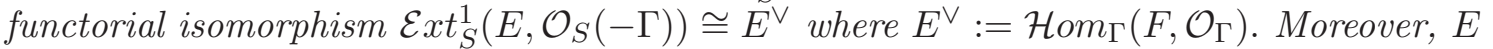
is reflexive, i.e. $\left(E^{\vee}\right)^{\vee} \cong E$.

Proof. This is [Hu-Le-1997, Prop 1.1.10] (or can easily be seen using Remark 3.2.1).

4.3.3. Irreducible components over non-reduced curves: sheaves of type II.

We now come back to $M_{C}, N_{C}, M_{\Delta}$ and $N_{\Delta}$ and turn to sheaves of type $I I$. We follow closely [Mo-2007, §3.1], which deals with the case of sheaves with even Euler charcteristics (those parametrized by $M$ ).

Lemma 4.3.7. Let $\mathcal{F}$ be a sheaf of type II supported on a curve $C=2 C^{\prime} \in \Delta$, let $\mathcal{F}_{\mid C^{\prime}}$ be its restriction to the reduced curve $C^{\prime}$, and let $F:=\mathcal{F}_{\mid C^{\prime}} / T$ be the quotient of $\mathcal{F}_{\mid C^{\prime}}$ by its torsion subsheaf $T:=\operatorname{Tors}\left(\mathcal{F}_{\mid C^{\prime}}\right)$. If $E$ is a pure dimension 1 sheaf on $S$ and $\alpha: \mathcal{F} \rightarrow E$ is a surjection which is not an isomorphism, then $E \cong F$ and $\alpha$ is a non zero multiple of the natural morphism $\mathcal{F} \rightarrow F$. As a consequence, $\mathcal{F}$ is (semi)stable if an only if

$$
\frac{\chi(\mathcal{F})}{2} \underset{(=)}{<} \chi(F) .
$$

Proof. Let $\alpha: \mathcal{F} \rightarrow E$ be a surjection onto a pure dimension 1 sheaf $E$. Then the Fitting support of $E$ is either $C$ or $C^{\prime}$. In the first case, $\operatorname{Ker} \alpha$ is supported on points, hence it has to be zero by the purity of $\mathcal{F}$. In particular, $\alpha$ is an isomorphism. It follows that we can consider only the case when the Fitting support of $E$ is $C^{\prime}$ and hence $E$ is an $\mathcal{O}_{C^{\prime}}$-module of rank one. Restricting $\alpha$ to $C^{\prime}$ we get a surjection $F \rightarrow E$, where $F:=\mathcal{F}_{\mid C^{\prime}} / \operatorname{Tors}\left(\mathcal{F}_{\mid C^{\prime}}\right)$, which has to be an isomorphism since $F$ and $E$ are torsion free sheaves of rank 1 on $C^{\prime}$. Using Definition 3.2.2, we conclude the proof.

Proposition 4.3.8. Let $C=2 C^{\prime} \in \Delta \subseteq B$ and let $\mathcal{F}$ be a sheaf parametrized by a point in $N_{C}^{I I}$ or in $M_{C}^{I I}$, i.e., a stable sheaf of type II (cf. Remark 4.3.2). Then $\mathcal{F}$ sits in the following exact sequence:

$$
0 \rightarrow F \otimes_{C^{\prime}} \mathcal{I} \rightarrow \mathcal{F} \rightarrow \mathcal{F}_{\mid C^{\prime}} \rightarrow 0
$$


where $F:=\mathcal{F}_{\mid C^{\prime}} / T$ and $T:=$ Tors $\left(\mathcal{F}_{\mid C^{\prime}}\right)$ is the torsion subsheaf of $\mathcal{F}_{\mid C^{\prime}}$. Furthermore, either $T=0$, which occurs if $\chi(\mathcal{F})$ is even (i.e. $\mathcal{F} \in M_{C}^{I I}$ ), or $T=\mathbb{C}_{p}$, for some point $p \in C^{\prime}$, which occurs if $\chi(\mathcal{F})$ is odd (i.e. $\left.\mathcal{F} \in N_{C}^{I I}\right)$.

Proof. In the even case, this is [Mo-2007, Lem. 3.1.6], whose proof we modify below to deal with the case of arbitrary $\chi$. Consider the exact sequence:

$$
\mathcal{F} \otimes_{C} \mathcal{I} \rightarrow \mathcal{F} \stackrel{r}{\rightarrow} \mathcal{F}_{\mid C^{\prime}} \rightarrow 0 .
$$

Since $\mathcal{I}$ is a nilpotent square zero ideal, $\mathcal{F} \otimes_{\mathcal{O}_{C}} \mathcal{I}$ is an $\mathcal{O}_{C^{\prime}}$-module, and $\mathcal{F} \otimes_{C} \mathcal{I} \cong$ $\mathcal{F}_{\mid C^{\prime}} \otimes_{\mathcal{O}_{C^{\prime}}} \mathcal{I}$. Since $\mathcal{F}$ is not of type $I$, the restriction map $r$ is not an isomorphism and $\operatorname{ker}(r)$ is a non-zero pure $\mathcal{O}_{C^{\prime}}$-module. This applies to the upcoming $K$ as well. We see that the sheaves $\operatorname{ker}(r)$ and of $\mathcal{F}_{\mid C^{\prime}}$ have first Chern class equal to $C^{\prime}$, so they are $\mathcal{O}_{C^{\prime}}$ modules of rank 1. Let $T:=\operatorname{Tor} s\left(\mathcal{F}_{\mid C^{\prime}}\right)$ be the torsion subsheaf of $\mathcal{F}_{\mid C^{\prime}}$. Then $F:=\mathcal{F}_{\mid C^{\prime}} \mid$ $T$ is torsion free of rank 1 . The natural surjective morphism $\mathcal{F}_{\mid C^{\prime}} \otimes_{\mathcal{O}_{C^{\prime}}} \mathcal{I} \rightarrow \operatorname{ker}(r)$ factors via the quotient $\mathcal{F}_{\mid C^{\prime}} \otimes_{\mathcal{O}_{C^{\prime}}} \mathcal{I} \rightarrow F \otimes_{\mathcal{O}_{C^{\prime}}} \mathcal{I}$, determining an isomorphism $F \otimes_{\mathcal{O}_{C^{\prime}}} \mathcal{I} \rightarrow \operatorname{ker}(r)$ of rank 1 torsion free sheaves on $C^{\prime}$. We can summarize this discussion in the following commutative diagram of short exact sequences (the zeros are omitted from the vertical ones):

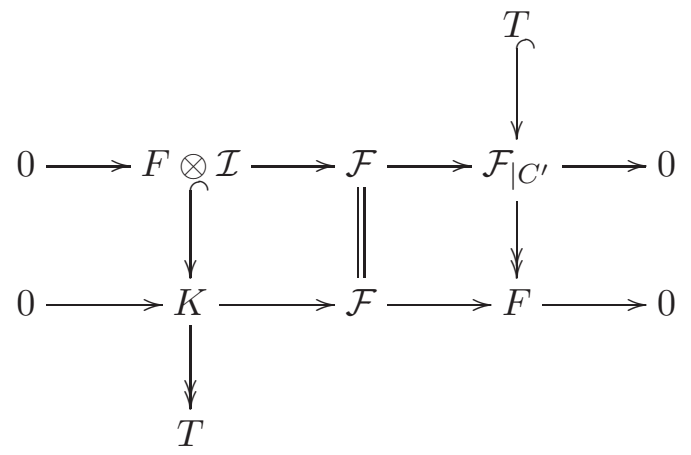

Using this diagram, together with (26), we get:

$$
\chi(\mathcal{F})=2 \chi(F)-2+\chi(T),
$$

showing that $\chi(\mathcal{F}) \equiv \chi(T)$ modulo 2. By using Lemma 4.3.7 to check stability, we see that:

$$
\frac{\chi(\mathcal{F})}{2}<\chi(F)
$$

so that $0 \leq \chi(T)<2$.

Corollary 4.3.9. Let $\mathcal{F}$ be a sheaf with Fitting support $C=2 C^{\prime}$ and odd Euler characteristic. Then $\mathcal{F}$ is not (the push forward of) a line bundle on $C$.

Proof. If $\mathcal{F}$ is of type $I$, then it is clear that it cannot be a locally free $\mathcal{O}_{C}$-module. If $\mathcal{F}$ is a sheaf of type $I I$, then by $(28), T \neq 0$ so $\mathcal{F}_{\mid C^{\prime}}$ is not locally free.

Proposition 4.3.10 (The case of $\chi$ even: the structure of $M_{C}^{I I}$ and $M_{\Delta}^{I I}$ ). Let $C=2 C^{\prime} \in \Delta \subseteq B$. The stable sheaves of type II on $C$ with even Euler characteristic 
$\chi=2 k$ are locally free. Restricting a sheaf to the underlying reduced curve defines a surjective morphism

$$
M_{C}^{I I} \rightarrow \mathrm{Pic}^{\mathrm{k}+2}\left(\mathrm{C}^{\prime}\right)
$$

which is a Zariski-locally trivial $\mathbb{C}^{3}$-bundle whose fibers are isomorphic to $H^{1}\left(C^{\prime}, \mathcal{I}\right)$. In particular, $M_{C}^{I I}$ is smooth and irreducible. The locally closed subvariety $M_{\Delta}^{I I}$ of $\widetilde{M}$ can be identified with degree- $(2 k+4)$ component $\operatorname{Pic}^{2 \mathrm{k}+4}\left(\mathcal{C}_{\Delta} / \Delta\right)$ of the relative Picard scheme of the family $\mathcal{C}_{\Delta} / \Delta$ of non-reduced curves. In particular, $M_{\Delta}^{I I}$ is smooth and irreducible.

Proof. The first step is to use [Mo-2007, Lemma 3.3.3]. We include the details to set up the notation for the case of $N_{C}^{I I}$, i.e., when $\chi$ is odd. Let $\mathcal{E}$ be any sheaf with Fitting support equal to $C$. Suppose $\mathcal{E}$ sits in the following exact sequence of $\mathcal{O}_{C}$-modules:

$$
0 \rightarrow E_{2} \rightarrow \mathcal{E} \rightarrow E_{1} \rightarrow 0
$$

where $E_{1}$ and $E_{2}$ are torsion free sheaves of rank one on $C^{\prime}$. The spectral sequence for the change of coefficients in the Ext groups [Mo-2007, Cor.3.2.2] gives:

$$
0 \rightarrow \operatorname{Ext}_{C^{\prime}}^{1}\left(E_{1}, E_{2}\right) \rightarrow \operatorname{Ext}_{C}^{1}\left(E_{1}, E_{2}\right) \stackrel{\delta}{\rightarrow} \operatorname{Hom}_{C^{\prime}}\left(E_{1} \otimes \mathcal{I}, E_{2}\right) \rightarrow \operatorname{Ext}_{C^{\prime}}^{2}\left(E_{1}, E_{2}\right) .
$$

Let $\varepsilon \in \operatorname{Ext}_{C}^{1}\left(E_{1}, E_{2}\right)$ be the class of this extension. By [Mo-2007, Lem 3.2.2], $E_{1}=$ $\mathcal{E} \otimes \mathcal{O}_{C} \mathcal{O}_{C^{\prime}}$ (equivalently, $E_{2}=\mathcal{I E}$ ) if and only if $\delta(\varepsilon): E_{1} \otimes \mathcal{I} \rightarrow E_{2}$ is surjective. Since $E_{1} \otimes \mathcal{I}$ is torsion free, this is the case if and only if $\delta(\varepsilon)$ is an isomorphism, which means that $E_{2} \cong E_{1} \otimes \mathcal{I}$. Moreover, by [Mo-2007, Prop 3.2.7] $\delta$ is surjective if and only if $E_{1}$ is locally free.

Now we consider the case of a stable sheave $\mathcal{F}$ with Fitting support equal to $C$ and even Euler characteristic. Then by diagram $(27) \mathcal{F}$ is an extension of $F$ by $F \otimes_{\mathcal{O}} \mathcal{I}$. So we set $E_{1}=F$ and $E_{2}=F \otimes_{\mathcal{O}} \mathcal{I}$ and the exact sequence (30) becomes:

$$
0 \rightarrow \operatorname{Ext}_{C^{\prime}}^{1}(F, F \otimes \mathcal{I}) \rightarrow \operatorname{Ext}_{C}^{1}(F, F \otimes \mathcal{I}) \stackrel{\delta}{\rightarrow} \operatorname{Hom}_{C^{\prime}}(F \otimes \mathcal{I}, F \otimes \mathcal{I})=\mathbb{C} .
$$

We conclude that if $\mathcal{F}$ is a sheaf in $M_{\Delta}^{I I}$, then $\delta$ is non zero, so it is surjective and hence $F$ is locally free. By using Lemma 3.3.1, $\mathcal{F}$ is locally free. Conversely, if $F$ is locally free, then $\operatorname{Ext}_{C^{\prime}}^{2}(F, F \otimes \mathcal{I})=H^{2}\left(C^{\prime}, F^{\vee} \otimes F \otimes \mathcal{I}\right)=0$, so $\delta$ is surjective and the isomorphism classes of $\mathcal{O}_{C}$-modules realized as extensions of $F$ by $F \otimes_{\mathcal{O}_{C^{\prime}}} \mathcal{I}$ are parametrized by the affine space $\mathbb{P}\left(\operatorname{Ext}_{C}^{1}(F, F \otimes \mathcal{I})\right) \backslash \mathbb{P}\left(\operatorname{Ext}_{C^{\prime}}^{1}(F, F \otimes \mathcal{I})\right)$. Since $F$ is locally free, $\operatorname{Ext}_{C^{\prime}}^{1}(F, F \otimes \mathcal{I}) \cong$ $H^{1}\left(C^{\prime}, \mathcal{I}\right)=\mathbb{C}^{3}$. If we set $\chi(\mathcal{F})=2 k$, by $(27)$ we get $\chi(F)=k+1$ and $\operatorname{deg}(F)=k+2$.

Associating to each $\mathcal{F}$ its restriction $F$ to the reduced underlying curve defines the morphism $M_{C}^{I I} \rightarrow \mathrm{Pic}^{\mathrm{k}+2}\left(\mathrm{C}^{\prime}\right)$. By the discussion above, the fibers are identified with the affine space $\mathbb{P}\left(\operatorname{Ext}_{C}^{1}(F, F \otimes \mathcal{I})\right) \backslash \mathbb{P}\left(\operatorname{Ext}_{C^{\prime}}^{1}(F, F \otimes \mathcal{I})\right) \cong H^{1}\left(C^{\prime}, \mathcal{I}\right)$. Another way of seeing that the fibers of the restriction morphism are isomorphic to $H^{1}\left(C^{\prime}, \mathcal{I}\right)$ is to consider the short exact sequence $0 \rightarrow \mathcal{I} \rightarrow \mathcal{O}_{C}^{\times} \rightarrow \mathcal{O}_{C^{\prime}}^{\times} \rightarrow 0$ and the corresponding sequence of first cohomology groups.

The last statement follows from the fact, proved above, that $\mathcal{F}$ is a locally free $\mathcal{O}_{C^{-}}$ module.

Corollary 4.3.11. Let $\mathcal{C}^{\prime} \rightarrow|H|=\Delta$ be the family of genus two curves. The kernel of the restriction morphism $\mathrm{Pic}_{\mathcal{C}_{\Delta} / \Delta}^{0} \rightarrow \mathrm{Pic}_{\mathcal{C}^{\prime} / \Delta}^{0}$ is an affine group scheme of relative dimension 3 , which acts trivially on the points of $M_{\Delta}^{I}$ and $N_{\Delta}^{I}$. 
Remark 4.3.12. The change of coefficients exact sequence (30) for the Ext-groups with respect to the closed embedding $C^{\prime} \subseteq C$, remains valid for the closed embedding $C \subset S$, thus giving an inclusion $\operatorname{Ext}_{C}^{1}\left(E_{1}, E_{2}\right) \subset \operatorname{Ext}_{S}^{1}\left(E_{1}, E_{2}\right)$. We claim that this is in fact an isomorphism. Indeed, any extension as $\mathcal{O}_{S}$-modules of $E_{1}$ by $E_{2}$ has Fitting support equal to $C$ and is automatically an $\mathcal{O}_{C}$-module. Moreover, any such extension is an extension of $\mathcal{O}_{C}$-modules.

We now deal with sheaves $\mathcal{F}$ of type $I I$ with odd Euler characteristic $\chi$, i.e. with $\mathcal{F} \in N_{\Delta}^{I I}$. The goal is to prove Proposition 4.3.16, the " $\chi$ odd" analogue of Proposition 4.3.10.

Consider a double curve $C \in \Delta$. In analogy with the case of even Euler characteristic treated earlier, in order to describe the open subvariety $N_{C}^{I I} \subset N_{C}$ we wish to realize every $\mathcal{F} \in N_{C}^{I I}$ as an extension of $\mathcal{O}_{C}$-modules by two rank 1 torsion free $\mathcal{O}_{C^{\prime}}$-modules. By looking at (27) and (28), we see that any $\mathcal{F} \in N_{C}^{I I}$ fits into a short exact sequence:

$$
0 \rightarrow K \rightarrow \mathcal{F} \rightarrow F \rightarrow 0 \text {. }
$$

with

$$
d:=\operatorname{deg}(F)=\frac{\chi+3}{2}, \quad \operatorname{deg}(K)=d-1 .
$$

As a consequence of Proposition 4.3.8, for each $\mathcal{F} \in N_{C}^{I I}$, we find a triple:

$$
(p, F, K) \in C^{\prime} \times \bar{J} a c^{d}\left(C^{\prime}\right) \times \bar{J} a c^{d-1}\left(C^{\prime}\right) .
$$

In the proof of Proposition 4.3.16 we will describe explicitly the set of triples occurring as above from a stable sheaf $\mathcal{F}$ of type $I I$ and odd Euler characteristic.

We start with the following two lemmata, relating the regularity properties of $F$ with those of $K$, and viceversa. These will only be relevant if the underlying reduced curve $C^{\prime}$ is singular, since otherwise $F$ and $K$ are clearly locally free.

Lemma 4.3.13. Let $\mathcal{F} \in N_{C}^{I I}$ be a stable sheaf of type II with odd Euler characteristic supported on a curve $C=2 C^{\prime}$, and let $(p, F, K)$ be the triple (33). If $F_{p}$ is free, then $F$ is locally free on $C^{\prime}$ and $K$ is locally free on $C^{\prime} \backslash p$.

Proof. From the first vertical sequence in (27) it follows that $K$ and $F$ are locally isomorphic away from $p$, so it is enough to prove the statement involving $F$. Suppose $F$ is locally free at $p$. Then $\operatorname{Ext}_{C^{\prime}}^{1}(F, T)=0$ so $\mathcal{F}_{\mid C^{\prime}} \cong F \oplus \mathbb{C}_{p}$. Using $(27)$ we get the following commutative diagram, with horizontal and vertical short exact sequences:

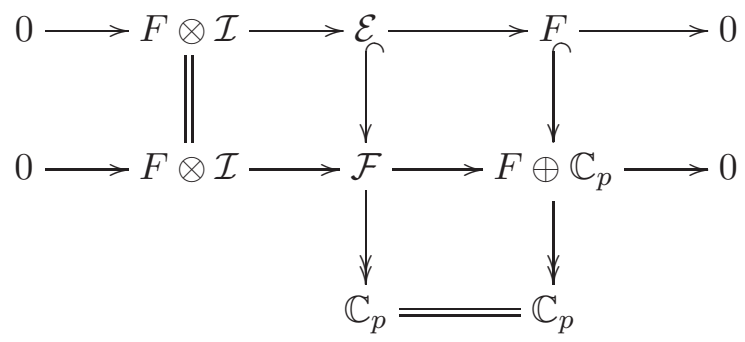

where $\mathcal{E}:=\operatorname{ker}\left[\mathcal{F} \rightarrow \mathbb{C}_{p}\right]$. Notice that $\mathcal{E}$, which is a pure sheaf of type $I I$, has even Euler characteristic. By Lemma 4.3.7 it follows that $F \cong \mathcal{E}_{\mid C^{\prime}} / \operatorname{Tors}\left(\mathcal{E}_{\mid C^{\prime}}\right)$. Using (29) we deduce 
that $\chi(\mathcal{E})=\chi(\mathcal{F})-1<2 \chi(F)$ and hence, by Lemma 4.3.7, $\mathcal{E}$ is stable. We may thus apply the arguments of the proof of Proposition 4.3.10 to $\mathcal{E}$ and deduce that $F$ is locally free.

By dualizing, we can reverse the role of $F$ and $K$.

Lemma 4.3.14. Let $\mathcal{F}$ and $(p, F, K)$ be as in Lemma 4.3.13. If $K_{p}$ is free, then $K$ is locally free on $C^{\prime}$, and $F$ is locally free on $C^{\prime} \backslash p$.

Proof. The sheaf $K$ is locally free at a point if and only if its dual $K^{\vee}:=\mathcal{H o m}_{C^{\prime}}\left(K, \mathcal{O}_{C^{\prime}}\right)$ is locally free at that point. It is therefore enough to prove the statement for $K^{\vee}$. Consider the short exact sequence (31). It can be viewed both as sequence of pure $\mathcal{O}_{C}$ and of $\mathcal{O}_{S^{-}}$ modules (cf. Remark 4.3.12). Applying $\mathcal{H o m}_{S}\left(-, \mathcal{O}_{S}\left(-C^{\prime}\right)\right.$ ) and using Lemma 4.3.6 we get:

$$
0 \rightarrow \mathcal{E} x t_{S}^{1}\left(F, \mathcal{O}_{S}\left(-C^{\prime}\right)\right) \rightarrow \mathcal{E} x t_{S}^{1}\left(\mathcal{F}, \mathcal{O}_{S}\left(-C^{\prime}\right)\right) \rightarrow \mathcal{E} x t_{S}^{1}\left(K, \mathcal{O}_{S}\left(-C^{\prime}\right)\right) \rightarrow 0 .
$$

where:

$\mathcal{E} x t_{S}^{1}\left(F, \mathcal{O}_{S}\left(-C^{\prime}\right)\right)=\mathcal{H o m}_{C^{\prime}}\left(F, \mathcal{O}_{C^{\prime}}\right)=: F^{\vee}, \mathcal{E} x t_{S}^{1}\left(K, \mathcal{O}_{S}\left(-C^{\prime}\right)=\mathcal{H o m}_{C^{\prime}}\left(K, \mathcal{O}_{C^{\prime}}\right):=K^{\vee}\right.$,

and:

$$
\mathcal{F}^{\prime}:=\mathcal{E} x t_{S}^{1}\left(\mathcal{F}, \mathcal{O}_{S}\left(-C^{\prime}\right)\right) \cong \mathcal{H o m}_{C}\left(\mathcal{F}, \mathcal{O}_{C}\right) \otimes_{\mathcal{O}_{S}} \mathcal{O}_{S}\left(-C^{\prime}\right)
$$

Notice that $\mathcal{F}^{\prime}$ is a pure sheaf of type $I I$, with odd Euler characteristic. By Lemma 4.3.7, up to multiplication by a non-zero scalar, the morphism $\mathcal{F}^{\prime} \rightarrow K^{\vee}$ is precisely the morphism $\mathcal{F}^{\prime} \rightarrow \mathcal{F}_{\mid C^{\prime}}^{\prime} /$ Tors $\left(\mathcal{F}_{\mid C^{\prime}}^{\prime}\right)$. Using (32) we find that:

$$
\chi\left(K^{\vee}\right)=-d, \quad \chi\left(F^{\vee}\right)=-d-1, \quad \chi\left(\mathcal{F}^{\prime}\right)=\chi\left(F^{\vee}\right)+\chi\left(K^{\vee}\right)=-2 d-1,
$$

so that we have:

$$
\frac{\chi\left(\mathcal{F}^{\prime}\right)}{2}<\chi\left(K^{\vee}\right)
$$

and by Lemma 4.3.7, $\mathcal{F}^{\prime}$ is stable. By Lemma 4.3 .15 below, the triple associated with $\mathcal{F}^{\prime}$, is precisely

$$
\left(p, K^{\vee}, F^{\vee}\right)
$$

By applying Lemma 4.3.13 to this triple, we see that if $K^{\vee}$ is locally free at $p$, then it is locally free everywhere.

We remark that in the proof of the lemma above we showed that the sheaf $\mathcal{F}^{\prime}$ defined in (35) is a stable sheaf of type $I I$ on $C$ with odd Euler characteristics. The following lemma describes how the triple (33) changes when passing from $\mathcal{F}$ to $\mathcal{F}^{\prime}$.

Lemma 4.3.15. Let $\mathcal{F}$ be as in Lemma 4.3.13. Let $\mathcal{F}^{\prime}=\mathcal{E} x t_{S}^{1}\left(\mathcal{F}, \mathcal{O}_{S}\left(-C^{\prime}\right)\right)$ be as in (35). Then the support of $T^{\prime}:=\operatorname{Tors}\left(\mathcal{F}_{\mid C^{\prime}}^{\prime}\right)$ is the same as the support $\{p\}$ of $T=\operatorname{Tors}\left(\mathcal{F}_{\mid C^{\prime}}\right)$. In particular, if $(p, F, K)$ is the triple (33) associated with $F$, then $\left(p, K^{\vee}, F^{\vee}\right)$ is the triple (33) associated with a sheaf $\mathcal{F}^{\prime}$ in $N_{C}^{I I}$ with $\chi\left(\mathcal{F}^{\prime}\right)=-2 d(F)-1$.

Proof. We have already observed in the course of proving Lemma 4.3.14 that $K^{\vee}=\mathcal{F}_{\mid C^{\prime}}^{\prime} /$ $T^{\prime}$ and that $F^{\vee}=\operatorname{ker}\left[\mathcal{F}^{\prime} \rightarrow K^{\vee}\right]$. Moreover, since $\mathcal{F}^{\prime}$ is stable of odd Euler characteristic, by Proposition 4.3.8, $T^{\prime}$ is a skyscraper sheaf supported at one point of $C^{\prime}$. We need to 
show that $\operatorname{Supp}\left(T^{\prime}\right)=\{p\}$. To this aim, consider the short exact sequences $0 \rightarrow F \otimes \mathcal{I} \stackrel{a}{\rightarrow}$ $K \rightarrow T \rightarrow 0$ and $0 \rightarrow K^{\vee} \otimes \mathcal{I} \stackrel{a^{\prime}}{\rightarrow} F^{\vee} \rightarrow T^{\prime} \rightarrow 0$ obtained by considering the left most column of (27) for the sheaves $\mathcal{F}$ and $\mathcal{F}^{\prime}$. We will show that $T$ and $T^{\prime}$ are supported on the same point by showing that the morphisms $a: F \otimes \mathcal{I} \rightarrow K$ and $a^{\prime} \otimes i d_{\omega_{C^{\prime}}}: K^{\vee} \rightarrow F^{\vee} \otimes \omega_{C^{\prime}}$ are dual to each other. Consider the short exact sequence:

$$
0 \rightarrow \mathcal{O}_{S}\left(-C^{\prime}\right) \stackrel{j}{\rightarrow} \mathcal{O}_{S} \rightarrow \mathcal{O}_{C^{\prime}} \rightarrow 0
$$

and its dual:

$$
0 \rightarrow \mathcal{O}_{S} \stackrel{j^{\vee}}{\rightarrow} \mathcal{O}_{S}\left(C^{\prime}\right) \rightarrow \mathcal{O}_{C^{\prime}}\left(C^{\prime}\right) \cong \omega_{C^{\prime}} \rightarrow 0 .
$$

Tensoring (36) by $\mathcal{F}$ we a morphism $\mathcal{F} \otimes_{\mathcal{O}_{S}} \mathcal{O}_{S}\left(-C^{\prime}\right) \rightarrow \mathcal{F}$ which by (27) factors as follows:

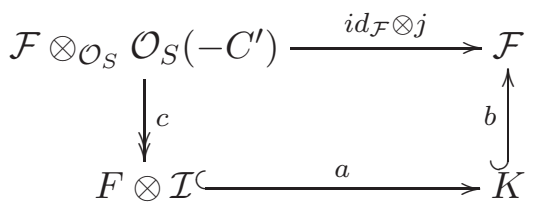

Similarly, we can tensor (36) by $\mathcal{F}^{\prime}$ and get a morphism $\mathcal{F}^{\prime} \otimes_{\mathcal{O}_{S}} \mathcal{O}_{S}\left(-C^{\prime}\right) \rightarrow \mathcal{F}^{\prime}$ which factors as:

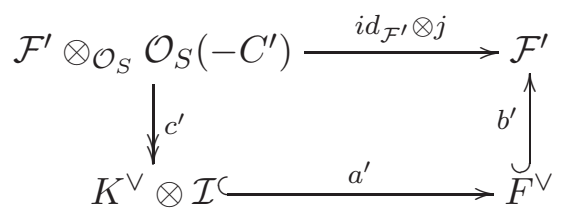

We can also dualize (38) by first applying $\mathcal{E} x t^{1}\left(-, \mathcal{O}_{S}\left(-C^{\prime}\right)\right)$ and then tensoring the resulting diagram by the line bundle $\mathcal{O}_{S}\left(-C^{\prime}\right)$. Using (34) and (35) we find:

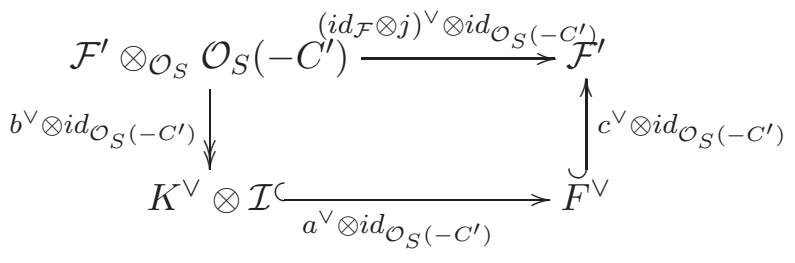

Using (36) - (37) we see that $\left(i d_{\mathcal{F}} \otimes j\right)^{\vee} \otimes i d_{\mathcal{O}_{S}\left(-C^{\prime}\right)}=i d_{\mathcal{F}^{\prime}} \otimes j^{\vee} \otimes i d_{\mathcal{O}_{S}\left(-C^{\prime}\right)}=i d_{\mathcal{F}^{\prime}} \otimes j$. It follows that diagrams (39) - (40) are two ways of factoring the same morphism into the product of three morphisms. By the first part of Lemma 4.3.7, the surjective morphism $b^{\vee} \otimes i d_{\mathcal{O}_{S}\left(-C^{\prime}\right)}: \mathcal{F}^{\prime} \otimes_{\mathcal{O}_{S}} \mathcal{O}_{S}\left(-C^{\prime}\right) \rightarrow K^{\vee} \otimes \mathcal{I}$ coincides (up to a scalar) with the morphism $c^{\prime}$ of diagram (39). Since this morphism is surjective, and the two morphism $b^{\prime}$ and $c^{\vee} \otimes i d_{\mathcal{O}_{S}\left(-C^{\prime}\right)}$ are injective, we may conclude that $a^{\vee} \otimes i d_{\mathcal{O}_{S}\left(-C^{\prime}\right)}=a^{\prime}$ and we are done.

We are now in the position to prove the " $\chi$ odd" analogue of Proposition 4.3.10.

Proposition 4.3.16 (The case of $\chi$ odd: the structure of $N_{C}^{I I}$ and $N_{\Delta}^{I I}$ ). Let $C=2 C^{\prime} \in \Delta \subseteq B$. The fiber $N_{C}^{I I}$ is irreducible. More precisely, the reduced underlying variety $\left(N_{C}^{I I}\right)_{\text {red }}$ is an affine bundle of rank 2 over a 3 -dimensional irreducible locally closed 
subvariety $Z \subset C^{\prime} \times \bar{J} a c^{d}\left(C^{\prime}\right) \times \bar{J} a c^{d-1}\left(C^{\prime}\right)$ parametrizing triples $(p, F, K)$ that arise from $\mathcal{F} \in N_{C}^{I I}$ as in (33). The fiber of this bundle over a point $(p, F, K)$ is given by:

$$
\mathbb{P} \operatorname{Ext}_{C}^{1}(F, K) \backslash \mathbb{P} \operatorname{Ext}_{C^{\prime}}^{1}(F, K),
$$

which is isomorphic to $\mathbb{C}^{2}$. Here, the inclusion $\operatorname{Ext}_{C^{\prime}}^{1}(F, K) \subset \operatorname{Ext}_{C}^{1}(F, K)$ inducing (41) is given as in (30) by the change of coefficients exact sequence.

Proof. By (27), every $\mathcal{F} \in N_{C}^{I I}$ is obtained as an extension of $F$ by $K$, so $\mathcal{F}$ determines an element in $\operatorname{Ext}_{C}^{1}(F, K)$. Conversely, the extensions in $\operatorname{Ext}_{C}^{1}(F, K) \backslash \operatorname{Ext}_{C^{\prime}}^{1}(F, K)$ (cf. (30)) correspond to stable sheaves of type $I I$ on $C$ with odd Euler characteristics. We now show that $N_{C}^{I I}$, with its reduced induced structure, is a fibration in affine spaces $\mathbb{P} \operatorname{Ext}_{C}^{1}(F, K) \backslash \mathbb{P} \operatorname{Ext}_{C^{\prime}}^{1}(F, K)$ over an irreducible variety $Z$ parametrizing the set of possible $F$ and $K$.

To see this, we start by computing $\operatorname{Ext}_{C}^{1}(F, K)$ and $\operatorname{Ext}_{C^{\prime}}^{1}(F, K)$. By Remark 4.3.12, $\operatorname{Ext}_{C}^{1}(F, K)=\operatorname{Ext}_{S}^{1}(F, K)$. The computations of both of these ext groups depend on the singularities of $C^{\prime}$, as well as on the local behavior of $F$ and $K$ at $p \in C^{\prime}$, or at any other singular point of $C^{\prime}$. In Lemmata 4.3.13 and 4.3.14, we ruled out some possibilities for the local behavior of the triples arising from a sheaf $\mathcal{F} \in N_{C}^{I I}$. In the proof of this proposition we will rule out one further case. We say that $F$ (or $K$ ) is singular at a point $p \in C^{\prime}$ if $F$ (respectively, $K$ ) is not locally free at $p$. We denote by $\operatorname{Sing}(F) \subset C^{\prime}$ the locus of points where $F$ is singular, and similarly for $K$. Throughout, we will use the following fundamental short exact sequence from $(27)$

$$
0 \rightarrow F \otimes \mathcal{I} \rightarrow K \rightarrow \mathbb{C}_{p} \rightarrow 0
$$

relating $F, K$, and $p$, where $\mathcal{I} \cong \omega_{C^{\prime}}^{\vee}$. Notice that (42) implies that $F$ and $K$ are locally isomorphic away from $p$. Using Lemma 4.3.3 we have the following relations between $F$ and $K$ in terms of their local behavior at $p$. Clearly, if $F$ and $K$ are both locally free, then $p$ has to be a smooth point of $C^{\prime}$ and we find:

$$
K \cong F \otimes \mathcal{I} \otimes \mathcal{O}_{C^{\prime}}(p)
$$

If $F$ is locally free, but $K_{p}$ is not free, then $K$ is uniquely determined:

$$
K \cong F \otimes \mathcal{I} \otimes m_{p}^{\vee}
$$

If $K$ is locally free, but $F_{p}$ is not free we find:

$$
F \cong K \otimes \mathcal{I}^{\vee} \otimes m_{p}
$$

If this is the case, then $K$ is not uniquely determined by $F$ and $p$. Indeed, by (2) Lemma 4.3.3, there is whole $\mathbb{P}^{1}=\mathbb{P} \operatorname{Ext}^{1}\left(\mathbb{C}_{p}, F \otimes \mathcal{I}\right.$ ) of possible (not mutually isomorphic) $K$ fitting in a short exact sequence as in (42). The locally free $K$ correspond to open subsets $\mathbb{C}^{*} \subset \mathbb{P}^{1}$, if $p$ is a node, or $\mathbb{C} \subset \mathbb{P}^{1}$, if $p$ is a cusp.

Finally, if neither $F_{p}$ nor $K_{p}$ are free, then we can write

$$
K=n_{*} G, F=n_{*} L, \text { with } G=L \otimes n^{*} \mathcal{I} \otimes \mathcal{O}_{\widehat{C^{\prime}}}(\widehat{p}),
$$

and where $n: \widehat{C^{\prime}} \rightarrow C^{\prime}$ is the normalization of $C^{\prime}$ at $\operatorname{Sing}(F)=\operatorname{Sing}(K)$, where $L$ and $G$ are locally free on $\widehat{C^{\prime}}$, and $\widehat{p} \in n^{-1}(p) \subset \widehat{C^{\prime}}$. In the course of the proof we will show that this last case does not occur if $F, K$, and $p$ arise from a stable sheaf of type $I I$. 
Lemma 4.3.17. Let $F, K$ and $p$ be as in (33). Then

$$
\operatorname{Ext}_{S}^{1}(F, K)=\left\{\begin{array}{c}
\mathbb{C}^{3} \\
\mathbb{C}^{4}
\end{array} \quad \text { if } F \text { or } K \text { are locally free on } C^{\prime} \backslash p\right.
$$

Proof. The Euler pairing (cf. [Hu-Le-1997, 6.1.5]) and Serre duality on $S$ yield:

$$
\begin{aligned}
\chi(F, K): & =\operatorname{dim} \operatorname{Hom}_{S}(F, K)-\operatorname{dim}_{\operatorname{Ext}_{S}^{1}}(F, K)+\operatorname{dim}_{\operatorname{Hom}_{S}}(K, F) \\
& =-v(F) \cdot v(K)=-C^{\prime 2}=-2 .
\end{aligned}
$$

By (32), $\operatorname{deg} F=\operatorname{deg} K+1$, so $\operatorname{Hom}_{S}(F, K)=\operatorname{Hom}_{C^{\prime}}(F, K)=0$. To prove the Lemma it is thus enough to show that $\operatorname{dim}_{\operatorname{Hom}_{C^{\prime}}}(K, F)=1$ if $F$ or $K$ are locally free away from $p$ and $\operatorname{dim} \operatorname{Hom}_{C^{\prime}}(K, F)=2$ otherwise. This is done using the expressions of equations (43), (44), (45), and (46). For example, if $F$ is locally free, then by (43) and (44), we have

$$
\operatorname{Hom}_{C^{\prime}}(K, F)=\operatorname{Hom}_{C^{\prime}}\left(F \otimes \mathcal{I} \otimes m_{p}^{\vee}, F\right)=H^{0}\left(C^{\prime}, \mathcal{I}^{\vee} \otimes m_{p}\right)=\mathbb{C} .
$$

A similar computation (using (45)) holds if $K$ is locally free, but $F_{p}$ is not free. If neither $F_{p}$ nor $K_{p}$ are free, then using (46) and Lemma 4.3 .5 we have

$$
\operatorname{Hom}_{C^{\prime}}(K, F)=\operatorname{Hom}_{\widehat{C^{\prime}}}\left(L \otimes n^{*} \mathcal{I} \otimes \mathcal{O}_{\widehat{C^{\prime}}}(\widehat{p}), L\right)=H^{0}\left(\widehat{C^{\prime}}, n^{*} \mathcal{I}^{\vee} \otimes \mathcal{O}_{\widehat{C^{\prime}}}(-\widehat{p})\right) .
$$

It follows that if $\widehat{C^{\prime}}$ is a curve of arithmetic genus 1 (i.e., $F$ and $K$ are locally free on $\left.C^{\prime} \backslash p\right)$, then $H^{0}\left(\widehat{C^{\prime}}, n^{*} \mathcal{I}^{\vee} \otimes \mathcal{O}_{\widehat{C^{\prime}}}(-\widehat{p})\right)=1$, while if $\widehat{C^{\prime}}$ is a smooth rational curve, then $H^{0}\left(\widehat{C^{\prime}}, n^{*} \mathcal{I}^{\vee} \otimes \mathcal{O}_{\widehat{C^{\prime}}}(-\widehat{p})\right)=2$.

Lemma 4.3.18. Let $F, K$ and $p$ as in (33). Then

$$
\operatorname{Ext}_{C^{\prime}}^{1}(F, K)=\left\{\begin{array}{cc}
\mathbb{C}^{2} & \text { if } F \text { or } K \text { is locally free } \\
\mathbb{C}^{3} & \text { if Sing }(F)=\text { Sing }(K)=\{p\} \\
\mathbb{C}^{4} & \text { otherwise }
\end{array}\right.
$$

Proof. We use the local to global spectral sequence, which yields the long exact sequence $0 \rightarrow H^{1}\left(C^{\prime}, \mathcal{H o m}_{C^{\prime}}(F, K)\right) \rightarrow \operatorname{Ext}_{C^{\prime}}^{1}(F, K) \rightarrow H^{0}\left(C^{\prime}, \mathcal{E}_{x} t_{C^{\prime}}^{1}(F, K)\right) \rightarrow H^{2}\left(C^{\prime}, \mathcal{H}_{C^{\prime}}(F, K)\right)=0$.

We thus have to compute $H^{1}\left(C^{\prime}, \mathcal{H o m}(F, K)\right)$ and $H^{0}\left(C^{\prime}, \mathcal{E} x t_{C^{\prime}}^{1}(F, K)\right)$. Using (43), (44), (45), and (46) we see that

$$
\mathcal{H o m}(F, K)=\left\{\begin{array}{cc}
\mathcal{I} \otimes m_{p}^{\vee} & \text { if } F_{p} \text { or } K_{p} \text { is free } \\
n_{*}\left(n^{*} \mathcal{I} \otimes \mathcal{O}_{\widehat{C^{\prime}}}(\widehat{p})\right) & \text { otherwise. }
\end{array}\right.
$$

This immediately implies that $H^{1}\left(C^{\prime}, \mathcal{H o m}(F, K)\right)=H^{1}\left(C^{\prime}, \mathcal{I} \otimes m_{p}^{\vee}\right)=\mathbb{C}^{2}$ if $F_{p}$ or $K_{p}$ is free; that $H^{1}\left(C^{\prime}, \mathcal{H o m}(F, K)\right)=H^{1}\left(\widehat{C}^{\prime}, n^{*} \mathcal{I} \otimes \mathcal{O}_{\widehat{C^{\prime}}}(\widehat{p})\right)=\mathbb{C}$ if $\operatorname{Sing}(F)=\operatorname{Sing}(K)=$ $\{p\}$ so $\widehat{C^{\prime}}$ is of arithmetic genus 1 ; and finally that $H^{1}\left(C^{\prime}, \mathcal{H o m}(F, K)\right)=H^{1}\left(\widehat{C^{\prime}}, n^{*} \mathcal{I} \otimes\right.$ $\left.\mathcal{O}_{\widehat{C^{\prime}}}(\widehat{p})\right)=0$ if $\# \operatorname{Sing}(F)=\# \operatorname{Sing}(K)=2$ so that $\widehat{C^{\prime}}$ is a smooth rational curve. To compute $H^{0}\left(C^{\prime}, \mathcal{E} x t_{C^{\prime}}^{1}(F, K)\right)$ we use Lemma 4.3 .4 to find:

$$
\mathcal{E} x t_{C^{\prime}}^{1}(F, K)=\bigoplus_{x \in \operatorname{Sing}(F)} \operatorname{Ext}_{\mathcal{O}_{C^{\prime}, x}}^{1}\left(F_{x}, K_{x}\right) \simeq \bigoplus_{x \in \operatorname{Sing}(F)} \mathbb{C}^{2}
$$

Putting everything together yields the proof of the Lemma. 
As a consequence of these two lemmas we see that the inclusion $\operatorname{Ext}_{C^{\prime}}^{1}(F, K) \subset \operatorname{Ext}_{S}^{1}(F, K)$ of Remark 4.3.12 is proper, i.e., there is a sheaf of type $I I$ on $C^{\prime}$ that is an extension of $K$ by $F$ if and only if $F$ and $K$ are locally free $C^{\prime} \backslash p$ and at most one is not locally free at $p$. When this is the case, then

$$
\operatorname{dim} \operatorname{Ext}_{C^{\prime}}^{1}(F, K)=2, \quad \operatorname{dim} \operatorname{Ext}_{S}^{1}(F, K)=3
$$

Now let

$$
Z \subset C^{\prime} \times \bar{J} a c^{d}\left(C^{\prime}\right) \times \bar{J} a c^{d-1}\left(C^{\prime}\right)
$$

be the set of such triples. The discussion above shows that $Z$ is contained in the open set $U \subset C^{\prime} \times \bar{J} a c^{d}\left(C^{\prime}\right) \times \bar{J} a c^{d-1}\left(C^{\prime}\right)$ consisting of points such that $F$ and $K$ are locally free $C^{\prime} \backslash p$ and at most one is not locally free at $p$. More precisely, we claim that $Z$ is the intersection of $U$ with the graph $\Gamma_{\psi}$ of the rational map

$$
\begin{aligned}
& C^{\prime} \times \bar{J} a c^{d}\left(C^{\prime}\right) \stackrel{\psi}{-} \bar{J} a c^{d-1}\left(C^{\prime}\right) \\
&(p, F) \longmapsto F \otimes \mathcal{I} \otimes m_{p}^{\vee} .
\end{aligned}
$$

Indeed, the fiber of the projection $\Gamma \rightarrow C^{\prime} \times \bar{J} a c^{d}\left(C^{\prime}\right)$ consists of: the sheaf $F \otimes \mathcal{I} \otimes m_{p}^{\vee}$ over the locus where $F$ is locally free at $p$; the isomorphism classes of extensions $\mathbb{P}^{1}=$ $\mathbb{P} \operatorname{Ext}_{C^{\prime}}^{1}\left(\mathbb{C}_{p}, F \otimes \mathcal{I}\right.$ ) over the points where $p \in \operatorname{Sing}(F)$ (cf. Lemma 4.3.3). It follows from (43), (44), (45), and from the remarks shortly thereafter, that $Z=\Gamma \cap U$. In particular, $Z$ is an irreducible variety of dimension 3 .

We are left with showing that the reduced fiber $\left(N_{C}^{I I}\right)_{\text {red }}$ is a 2-dimensional affine bundle over $Z$, with fiber $\mathbb{P} \operatorname{Ext}_{S}^{1}(F, K) \backslash \mathbb{P} \operatorname{Ext}_{C^{\prime}}^{1}(F, K)$ over a point $(p, F, K)$. To see this, first note that if $\mathcal{F}_{W}$ is flat family of sheaves in $N_{C}^{I I}$, parametrized by a reduced scheme $W$, then we can preform the construction of Proposition 4.3.8 in families to get $W$-flat families $F_{W}$ and $K_{W}$, of rank 1 torsion free sheaves of degree $d$ and $d-1$, respectively, as well as a flat family of degree one skyscraper sheaves $T_{W}$. By the universal property of compactified Jacobians, these families induce a regular morphism $W \rightarrow C^{\prime} \times \bar{J} a c^{d}\left(C^{\prime}\right) \times \bar{J} a c^{d-1}\left(C^{\prime}\right)$ whose image is contained in $Z$. Now choose as $W$ the preimage of $N_{C}^{I I}$ in the appropriate Quot scheme, taken with its reduced induced structure. Then $W$ is such that its quotient by the group action on the Quot scheme is exactly $\left(N_{C}^{I I}\right)_{\text {red }}$ (cf. [Mu, (3) pg 29]). The equivariant morphism $W \rightarrow Z$ factors via the quotient morphism $W \rightarrow\left(N_{C}^{I I}\right)_{\text {red }}$ and induces a surjective morphism $\left(N_{C}^{I I}\right)_{\text {red }} \rightarrow Z$. By (50), the fibers of this morphism are affine 2-dimensional spaces.

This ends the proof of Proposition 4.3.16

Remark 4.3.19. By [Ch-Ka-2016, Lem. 4.12], the varieties $N_{C}^{I I}$ and $N_{\Delta}^{I I}$ are non-reduced.

\subsection{The top degree direct image sheaves $R^{10}$ and the local systems $\mathscr{L}$.}

The purpose of this section is to prove Proposition 4.4.3 which describes the restriction of the sheaves $R^{10} \widetilde{m}_{*} \mathbb{Q}$ and $R^{10} n_{*} \mathbb{Q}$ to the locally closed subvarieties $B \backslash \Sigma, \Sigma \backslash \Delta$ and $\Delta$ of $B$. The proof of the proposition uses: the analysis of the irreducible components of the restrictions of $\widetilde{M}, M$ and $N$ to the loci $B \backslash \Sigma, \Sigma \backslash \Delta$ and $\Delta$ and of the corresponding fibers (Proposition 4.2.4); some basic properties of the trace morphism and of the direct image sheaf in top degree, as summarized in Fact 4.4.1; the general topological Lemma 4.4.2. 
Fact 4.4.1. (Trace morphism and direct image in top degree) In this section, we need the following three sets of facts:

(1) The basic properties of the trace morphism $\operatorname{Tr}_{f}: R^{2 d} f_{!} \mathbb{Q}_{X}(d) \rightarrow \mathbb{Q}_{T}$, for a flat morphism $f: X \rightarrow T$ of relative dimension d. See [SGA 4.3], especially Exp. XVIII, Théorème 2.9 (functoriality and compatibility properties), and Remarque 2.10 .1 (the trace morphism is an isomorphism if and only if all the fibers of $f$ have a unique irreducible component of dimension d).

(2) If a flat morphism $f: X \rightarrow T$ of relative dimension $d$ has reduced fibers, then the sheaf $R^{2 d} f_{!} \mathbb{Q}_{X}(d)$ is the $\mathbb{Q}_{T}$-linearization of the sheaf of sets of irreducible components of the fibers of $f$; see [Ngô-2008, Proposition 7.3.2].

(3) Let $f: X \rightarrow T$ be a projective morphism of pure relative dimension $d$ and with irreducible fibers. Let $L^{d}: \mathbb{Q}_{X} \rightarrow \mathbb{Q}_{X}[2 d]$ be the morphism induced by the $d$-th power of the first Chern class of an $f$-ample line bundle $L$ on $X$. In view of the irreducibility of the fibers, by pushing forward, we get an isomorphism $L^{d}: \mathbb{Q}_{T} \stackrel{\sim}{\rightarrow}$ $R^{2 d} f_{*} \mathbb{Q}_{X}$.

Moreover, if $U \subset X$ is a Zariski open subset intersecting every fiber of $f$ and $f_{U}: U \rightarrow T$ is the restriction of $f$ then $R^{2 d} f_{U !} \mathbb{Q}_{X} \simeq R^{2 d} f_{*} \mathbb{Q}_{X} \simeq \mathbb{Q}_{T}$. In fact, by denoting by $f_{X \backslash U}: X \backslash U \rightarrow T$ the restriction of $f$, the statement follows from the exact sequence:

$$
R^{2 d-1} f_{X \backslash U *} \mathbb{Q}_{X \backslash U}=0 \longrightarrow R^{2 d} f_{U !} \mathbb{Q}_{U} \stackrel{R^{2 d} j_{!}}{\simeq} R^{2 d} f_{*} \mathbb{Q}_{X} \longrightarrow 0=R^{2 d} f_{X \backslash U *} \mathbb{Q}_{X \backslash U},
$$

where the vanishing statements hold since the fibers of $f_{X \backslash U}$ have at most dimension $d-1$.

Lemma 4.4.2. Let $X$ be a variety of pure dimension $d+n$, let $T$ be a normal irreducible variety of dimension $n$ and let $f: X \rightarrow T$ be a projective surjective morphism.

Let $\emptyset=X_{0} \subset X_{1} \subset X_{2} \subset \cdots \subset X_{m-1} \subset X_{m}=X$ be a filtration of $X$ by closed subvarieties such that, for $0<i \leq m$ :

(1) Every fiber of the restriction $f_{i}^{0}: X_{i} \backslash X_{i-1} \rightarrow T$ of $f$ has dimension $d$,

(2) $R^{2 d} f_{i !}^{0} \mathbb{Q}_{X_{i} \backslash X_{i-1}} \simeq \mathbb{Q}_{T}$.

Then the direct image sheaf in top degree $R^{2 d} f_{*} \mathbb{Q}_{X}$ is the trivial local system of rank $m$. Proof. Without loss of generality, we may assume that the $X_{i}$ are reduced. Let $f_{i}: X_{i} \rightarrow T$ be the restriction of $f$.

CLAIM: the sheaves $R^{2 d} f_{i !} \mathbb{Q}_{X_{i}}$ are local systems of rank $i$.

We now prove the CLAIM by induction on $i$. Let $\iota: X_{i-1} \rightarrow X_{i} \leftarrow X_{i} \backslash X_{i-1}: j$ be the closed and open complementary embeddings. By applying $R f_{i \text { ! }}$ to the short exact sequence $0 \rightarrow j_{!} \mathbb{Q}_{X_{i} \backslash X_{i-1}} \rightarrow \mathbb{Q}_{X_{i}} \rightarrow i_{*} \mathbb{Q}_{X_{i-1}} \rightarrow 0$, we get the following long exact sequence:

$$
R^{2 d-1} f_{i-1 *} \mathbb{Q}_{X_{i-1}} \longrightarrow R^{2 d} f_{i !}^{0} \mathbb{Q}_{X_{i} \backslash X_{i-1}} \stackrel{R^{2 d} j !}{\longrightarrow} R^{2 d} f_{i *} \mathbb{Q}_{X_{i}} \longrightarrow R^{2 d} f_{i-1 *} \mathbb{Q}_{X_{i-1}} \longrightarrow 0 .
$$

By (2), we have that $R^{2 d} f_{i !}^{0} \mathbb{Q}_{X_{i} \backslash X_{i-1}} \simeq \mathbb{Q}_{T}$. By the induction hypothesis, we have that $R^{2 d} f_{i-1 *} \mathbb{Q}_{X_{i-1}} \simeq \mathbb{Q}_{T}^{i-1}$. It follows that, if $R^{2 d} j_{\text {! }}$ is an injective morphism of constructible sheaves, then $R^{2 d} f_{i *} \mathbb{Q}_{X_{i}}$ is a rank $i$ local system. In this case, the CLAIM would follow. 
Since $R^{2 d} f_{i !}^{0} \mathbb{Q}_{X_{i} \backslash X_{i-1}}$ is a local system it suffices to check the desired injectivity on a non-empty open subset of the irreducible base $T$. Shrinking $T$ if necessary, we may assume that $X_{i-1}$ and $X_{i}$ are flat over $T$ : in this case it makes sense to consider the trace morphisms $\operatorname{Tr}_{f_{i}^{0}}: R^{2 d} f_{i !}^{0} \mathbb{Q}_{X_{i} \backslash X_{i-1}} \rightarrow \mathbb{Q}_{T}$ and $\operatorname{Tr}_{f_{i}}: R^{2 d} f_{i *} \mathbb{Q}_{X_{i}} \rightarrow \mathbb{Q}_{T}$. By [SGA 4.3], Exp. XVIII, Théorème 2.9 (basic functoriality and compatibility properties), we have $\operatorname{Tr}_{f_{i}^{0}}=\operatorname{Tr}_{f_{i}} \circ R^{2 d} j_{!}$. Moreover $R^{2 d} f_{i !}^{0} \mathbb{Q}_{X_{i} \backslash X_{i-1}} \simeq \mathbb{Q}_{T}$ implies, by base change, that every fiber of $f_{i}^{0}$ has a unique irreducible component of top dimension $d$. By [SGA 4.3, Exp. XVIII, Remarque 2.10.1 ], the trace morphism $\operatorname{Tr}_{f_{i}^{0}}$ is an isomorphism. We conclude, in particular, that the morphism $R^{2 d} j_{\text {! }}$ must be injective. The CLAIM is proved.

The conclusion follows from the fact that the morphism $\operatorname{Tr}_{f_{i}^{0}}^{-1} \circ \operatorname{Tr}_{f_{i}}$ splits the short exact sequence stemming from (51).

Proposition 4.4.3. (The direct image sheaves in top degree) Let $R_{M}^{10}:=R^{10} m_{*} \mathbb{Q}$,, $R_{\widetilde{M}}^{10}:=R^{10} \widetilde{m}_{*} \mathbb{Q}$, and $R_{N}^{10}:=R^{10} n_{*} \mathbb{Q}$, Then we have canonical isomorphisms of constructible sheaves:

$$
\begin{array}{lll}
R_{M \mid B \backslash \Delta}^{10} \simeq \mathbb{Q}_{B \backslash \Sigma}, & R_{M \mid \Delta}^{10} \simeq \mathbb{Q}_{\Delta}^{\oplus 2} ; \\
R_{\widetilde{M} \mid B \backslash \Sigma}^{10} \simeq \mathbb{Q}_{B \backslash \Sigma}, & R_{\widetilde{M} \mid \Sigma \backslash \Delta}^{10} \simeq \mathbb{Q}_{\Sigma \backslash \Delta}^{\oplus 2}, & R_{\widetilde{M} \mid \Delta}^{10} \simeq \mathbb{Q}_{\Delta}^{\oplus 4} ; \\
R_{N \mid B \backslash \Sigma}^{10} \simeq \mathbb{Q}_{B \backslash \Sigma} & R_{N \mid \Sigma \backslash \Delta}^{10} \simeq \mathbb{Q}_{\Sigma \backslash \Delta} \oplus \mathscr{L}_{\Sigma \backslash \Delta}, & R_{N \mid \Delta}^{10} \simeq \mathbb{Q}_{\Delta}^{\oplus 2},
\end{array}
$$

where $\mathscr{L}$ is the rank one local system on $\Sigma \backslash \Delta$ corresponding to the $\mathbb{Z} / 2 \mathbb{Z}$-module of character -1 via the isomorphism $\pi_{1}(\Sigma \backslash \Delta) \simeq \mathbb{Z} / 2 \mathbb{Z}$.

Proof. For ease of notation: if $W$ is a variety, $\varphi: W \rightarrow B$ is a morphism and $Z \subset B$ is a subvariety, then we set $W_{Z}:=\varphi^{-1}(Z)$ and $R_{W}^{10}:=R^{10} \varphi_{!} \mathbb{Q}_{W}$.

The statements in the first column of (52) follow from the fact that the morphisms in question are flat of relative dimension five with integral fibers over the indicated loci; see Remark 3.2.6 and Proposition 4.2.2.

Proof that $R_{\widetilde{M} \mid \Sigma \backslash \Delta}^{10} \simeq \mathbb{Q}_{\Sigma \backslash \Delta}^{\oplus 2}$. Recall from Lemma 4.1.3 and proper base change that

$$
\left(R b_{*} \mathbb{Q}_{\widetilde{M}}\right)_{\mid M_{\Sigma \backslash \Delta}} \simeq \mathscr{I} \mathscr{C}_{M_{\Sigma \backslash \Delta}} \oplus \mathbb{Q}_{\left(\mathrm{Sym}^{2} M^{\prime}\right)_{\Sigma \backslash \Delta}}[-2] .
$$

By [Le-So-2006], the singularities of $M_{\Sigma \backslash \Delta}$ are of type $A_{1}$ quotient singularities, so that

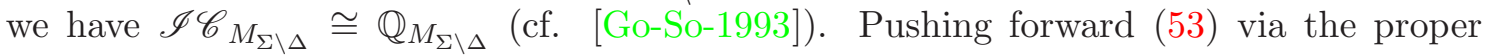
morphism $m: M_{\Sigma \backslash \Delta} \rightarrow \Sigma \backslash \Delta$ we get:

$$
R \widetilde{m}_{*} \mathbb{Q}_{\widetilde{M}_{\Sigma \backslash \Delta}}=R m_{*} R b_{*} \mathbb{Q}_{\widetilde{M}_{\Sigma \backslash \Delta}}=R m_{*} \mathbb{Q}_{M_{\Sigma \backslash \Delta}} \oplus R p_{*}^{2} \mathbb{Q}_{\left(\mathrm{Sym}^{2} M^{\prime}\right)_{\Sigma \backslash \Delta}}[-2] .
$$

and thus:

$$
R^{10} \widetilde{m}_{*} \mathbb{Q}_{\widetilde{M}_{\Sigma \backslash \Delta}}=R^{10} m_{*} \mathbb{Q}_{M_{\Sigma \backslash \Delta}} \oplus R^{8} p_{*}^{2} \mathbb{Q}_{\mathrm{Sym}^{2} M_{\Sigma \backslash \Delta}^{\prime}}
$$


By using Fact 4.4.1.(2) and the irreducibility of the five dimensional $M_{b}$ (cf. Proposition 4.2.2) and of the four dimensional $M_{b}^{\prime}$ (cf. Remark 4.1.2), we see that $R^{10} m_{*} \mathbb{Q}_{M_{\Sigma \backslash \Delta}}=$ $\mathbb{Q}_{\Sigma \backslash \Delta}(-5)$ and $R^{8} p_{*}^{2} \mathbb{Q}_{\mathrm{Sym}^{2} M_{\Sigma \backslash \Delta}^{\prime}} \cong \mathbb{Q}_{\Sigma \backslash \Delta}(-4)$ and we are done.

Proof that $R_{N_{\Sigma \backslash \Delta}}^{10} \simeq \mathbb{Q}_{\Sigma \backslash \Delta} \oplus \mathscr{L}_{\Sigma \backslash \Delta}$. The variety $N_{\Sigma \backslash \Delta}$ is irreducible (cf. [Ra-2008, Proof of Prop 1.3]), the morphism $N_{\Sigma \backslash \Delta} \rightarrow \Sigma \backslash \Delta$ is flat, with each of its fibers are reduced and with two irreducible components (cf. Proposition 4.2.2). The sheaf $R_{N_{\Sigma \backslash \Delta}}^{10}$ is the $\mathbb{Q}$-linearization of the sheaf of sets $\mathfrak{I}$ of irreducible components of $N_{\Sigma \backslash \Delta} /(\Sigma \backslash \Delta)$. Let $V \subseteq N_{\Sigma \backslash \Delta}$ be the locus parameterizing line bundles. By Proposition 4.2.2 and its proof we have that: the morphism $V \rightarrow \Sigma \backslash \Delta$ is surjective and smooth; $V$ is dense in each fiber $N_{b} ; V_{b}$ has two connected components. It follows that the sheaf of sets $\mathfrak{I}$ is locally constant, with stalks of cardinality two. We now examine the monodromy of $\mathfrak{I}$ and relate it to that of the broken curves. Let $b \in \Sigma \backslash \Delta$. The corresponding curve $C_{b}=C_{1, b}+C_{2, b} \in|2 H|$ has two irreducible components. The two connected components of $V_{b}$ parameterize line bundles of bi-degree $(2,3)$ and $(3,2)$ (cf. Lemma 3.3.2). Since $\Sigma \backslash \Delta$ is nonsingular and connected, the monodromy can be detected on a Zariski dense open subset $U \subseteq \Sigma \backslash \Delta$. We take $U:=\operatorname{Sym}^{2} B^{\prime o}$, where $B^{\prime o} \subseteq B^{\prime}=|H|$ is the locus of nonsingular curves. It is clear that looping in $U$ around the diagonal exchanges the two components of $C_{b}$, the same way in which the two lines of the corresponding broken conic are. It follows that the bidegrees are swapped as well. The desired conclusion follows.

Proof of the remaining isomorphisms. We now focus on the third column, of (52), i.e. on the situation over $\Delta$. By proper base change we need to show that: (a) $R_{M_{\Delta}}^{10} \simeq \mathbb{Q}_{\Delta}^{2}$; (b) $R_{\bar{M}_{\Delta}}^{10} \simeq \mathbb{Q}_{\Delta}^{4}$; (c) $R_{N_{\Delta}}^{10} \simeq \mathbb{Q}_{\Delta}^{2}$.

We are going to use Lemma 4.4.2 with filtrations of length two in cases (a) and (c) and of length four in case (b).

We first deal with cases (a) and (c). In case $(a)$ and (c), we choose the first space $X_{1}$ of the filtration to be the locus of rank two semistable sheaves (cf. Proposition 4.3.1), i.e. in case (a), we set $X_{1}:=M_{\Delta}^{I}$, in case (c), we set $X_{1}:=N_{\Delta}^{I}$. By Proposition 4.3.1, $M_{\Delta}^{I}$ and $N_{\Delta}^{I}$ are projective over $\Delta$ with 5-dimensional irreducible fibers. By Fact 4.4.1.(3), we have that $R_{M_{\Delta}^{I}}^{10} \simeq \mathbb{Q}_{\Delta} \simeq R_{N_{\Delta}^{I}}^{10}$. Note that with our choice of $X_{1}$, we have that $X_{2} \backslash X_{1}$ is, $M_{\Delta}^{I I}$ in case $(a), N_{\Delta}^{I I}$ in case $(c)$. By Proposition 4.2.4 (the irreducibility of the fibers of $M_{C^{\prime}}^{I I}$ and $N_{C^{\prime}}^{I I}$ ), and by Fact 4.4.1.(1), we are thus in the position of applying Lemma 4.4.2 and reach the desired conclusion in cases (a) and (c).

In order to prove (b) via an application of Lemma 4.4.2, we consider the following increasing filtration of $\widetilde{M}_{\Delta}$ into closed subvarieties:

$$
X_{1}:=E_{\Delta}^{I} \subseteq X_{2}:=E_{\Delta} \subseteq X_{3}:=b^{-1}\left(M_{\Delta}^{I}\right) \subseteq X_{4}:=\widetilde{M}_{\Delta}
$$

Recall Proposition 4.2.1 and the subvarieties $E_{\Delta}=E_{\Delta}^{I} \amalg E_{\Delta}^{I I}$ defined in (24). By using that $b$ is an isomorphism away from the center $\operatorname{Sym}^{2} M^{\prime}$ of the blow up, the successive differences are seen to be as follows:

$$
X_{2} \backslash X_{1}=E_{\Delta}^{I I}, \quad X_{3} \backslash X_{2}=M_{\Delta}^{I} \backslash \operatorname{Sym}_{\Delta}^{2} M^{\prime}, \quad X_{4} \backslash X_{3}=M_{\Delta}^{I I}
$$


As shown in Proposition 4.2.1, the projective variety $X_{1}=E_{\Delta}^{I}$ has irreducible fibers of dimension 5 over $\Delta$ : by Fact 4.4.1.(3) we get $R_{E_{\Delta}^{I}}^{10} \simeq \mathbb{Q}_{\Delta}$.

By Proposition 4.2.1, the variety $X_{2} \backslash X_{1}=E_{\Delta}^{I I}$ is flat surjective over $\Delta$ with irreducible fibers. By Fact 4.4.1.(1), we get that $R_{E_{\Delta}^{I I} \backslash E_{\Delta}^{I}} \simeq \mathbb{Q}_{\Delta}$.

By Remark 4.1.2, the fibers of $M_{\Delta}^{I}$ and of $X_{3} \backslash X_{2}$ over the points of $\Delta$ are non empty and irreducible of dimension five. By Fact 4.4.1.(3), we conclude that $R_{M_{\Delta}^{I} \backslash\left(\operatorname{Sym}^{2} M^{\prime}\right)_{\Delta}}^{10} \simeq \mathbb{Q}_{\Delta}$.

The morphism $M_{\Delta} \rightarrow \Delta$ is flat, so that its restriction to the open subset $M_{\Delta}^{I I} \subset M_{\Delta}$ is also flat. By Proposition 4.2.4, the fibers of $M_{\Delta}^{I I} \rightarrow \Delta$ are irreducible of dimension five. By Fact 4.4.1.(1), we have that $R_{M_{\Delta}^{I I}}^{10} \simeq \mathbb{Q}_{\Delta}$. We conclude that (b) holds in view of Lemma 4.4.2.

Remark 4.4.4. (Properties of $\mathscr{L}$ in (52)) We record the following elementary properties of $\mathscr{L}$. Let $T$ be an irreducible normal variety of positive dimension, let $\mathscr{L}_{T}$ be the rank one local system on $\operatorname{Sym}^{2} T \backslash \Delta_{T}$ corresponding to the $\mathbb{Z} / 2 \mathbb{Z}$ module of character -1 via the epimorphism $\pi_{1}\left(\operatorname{Sym}^{2} T \backslash \Delta_{T}\right) \rightarrow \mathbb{Z} / 2 \mathbb{Z}$. Let $j: U \rightarrow \operatorname{Sym}^{2} T \backslash \Delta_{T}$ be any open immersion with $U \neq \emptyset$, and let $i: \operatorname{Sym}^{2} T \backslash \Delta_{T} \rightarrow \operatorname{Sym}^{2} T$ be the evident open immersion. Let $u: T \rightarrow T^{\prime}$ be a non constant morphism of irreducible normal varieties. Let $u^{(2)}: \operatorname{Sym}^{2} T \rightarrow \operatorname{Sym}^{2} T^{\prime}$ and $u^{(2)^{\prime}}: \operatorname{Sym}^{2} T \backslash u^{(2)}{ }^{-1}\left(\Delta_{T^{\prime}}\right) \rightarrow \operatorname{Sym}^{2} T^{\prime} \backslash \Delta_{T^{\prime}}$ be the resulting evident morphisms. Then:

(1) $i_{*} \mathscr{L}=i_{!} \mathscr{L}=R i_{1} \mathscr{L}=R i_{*} \mathscr{L}$;

(2) $j_{*} j^{*} \mathscr{L}=\mathscr{L}$;

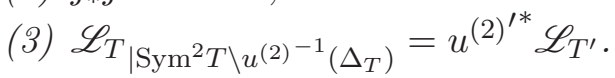

As to (1): the first $=$ is because there are no local invariants around the diagonal; the second $=i$ s because $i$ is an open immersion; the third equality follows from Verdier duality and the self-duality of $\mathscr{L}$. As to (2): in view of the normality assumption, it is valid for any local system, due to the surjection of fundamental groups $\pi_{1}(U, u) \rightarrow \pi_{1}\left(\operatorname{Sym}^{2} T \backslash \Delta_{T}\right)$. As to (3): this is because the natural surjection of fundamental groups above for $T$ factors through the one for $T^{\prime}$.

Finally, for every integer $\mathfrak{d} \in \mathbb{Z}$, when $T$ is irreducible nonsingular, the local system $\mathscr{L}$ underlies a unique structure of polarizable pure Hodge-Tate variation of Hodge structures of type $(\mathfrak{d}, \mathfrak{d}) ;$ see $\S 2.1$.

\section{The Ngô Strings of Several Lagrangian fibrations}

The aim of this section is to determine the Ngô strings (cf. Definition 7.0.4) of the two Lagrangian fibrations $\widetilde{M}, N \rightarrow B$ as well as of several other auxiliary fibrations. In $\S 5.1$ we introduce the Ngô strings that will appear in the Decomposition Theorem for $\widetilde{M} \rightarrow B$ and $N \rightarrow B$. Then in $\S 5.2$ and $\S 5.3$ we determine the Decomposition Theorem for the two universal families of curves of the linear systems $|H|$ and $|2 H|$ on the K3 surface. To prove Theorems A, B, and B', we need to compute the cohomology of the Ngô strings appearing the Decomposition Theorem for $\widetilde{M}, N \rightarrow B$. We do by realizing them as direct summands of the Decomposition theorem for other Lagrangian fibrations whose cohomology is known. This is done in this in $\S 5.4, \S 5.5$, and $\S 5.6$. Finally, in $\S 5.7$ we prove 
that the Ngô strings (60) are precisely those appearing in Decomposition Theorem for the two Lagrangian fibrations. This builds on $\S 5.2$ and $\S 5.3$.

All decomposition-type results that follow take place in the categories $D^{b} M H M_{a l g}(-)$ and have an evident counterpart (turn off the Tate shifts) in the corresponding categories $D^{b}(-, \mathbb{Q})$ via the functor rat. We only state the stronger result in $D^{b} M H M_{\text {alg }}(-)$.

\subsection{Some relevant strings.}

Let $u: \mathscr{C} \rightarrow \mathscr{B}$ be a family of nonsingular projective, not necessarily connected, curves of genus $g$. Let $v: \mathscr{P} \rightarrow \mathscr{B}$ be the relative $\operatorname{Pic}_{\mathscr{C} / \mathscr{B}}^{0}$. We consider the higher direct image sheaves $\Lambda_{\mathscr{B}}^{\bullet}:=R^{\bullet} v_{*} \mathbb{Q}_{\mathscr{P}}$, with $\bullet \in[0,2 g]$; these are polarizable variations of pure Hodge structures of weight $\bullet$. By taking into account Tate twists, we have natural isomorphisms:

$$
R^{1} u_{*} \mathbb{Q}_{\mathscr{C}}=R^{1} v_{*} \mathbb{Q}_{\mathscr{P}} ; \quad \Lambda_{\mathscr{B}}^{\bullet}=\wedge^{\bullet} R^{1} u_{*} \mathbb{Q}_{\mathscr{C}}, \quad \Lambda_{\mathscr{B}}^{g-\bullet}=\Lambda_{\mathscr{B}}^{g+\bullet}(\bullet)
$$

We come back to our families of curves $\mathcal{C}^{\prime} \rightarrow B^{\prime}$ and $\mathcal{C} \rightarrow B$ over the linear systems $B^{\prime}=|H|$ and $B=|2 H|$. By restricting these two families over the respective sets of regular values $B^{\prime o}$ and $B^{o}$, we obtain families of type $u$ as above, of genus 2 and 5 respectively, and with connected fibers. By first restricting the family of curves $\mathcal{C} \rightarrow B$ over $\Sigma^{o}:=$ $\operatorname{Sym}^{2} B^{\prime o} \backslash \Delta_{B^{\prime}}$, and then by normalizing the total space of the resulting family, we obtain a family of type $u$ as above, with connected total space, but with disconnected fibers given by the disjoint union of two curves of genus 2. We thus have (cf. (20)):

$$
\begin{array}{ccc}
\mathcal{C}_{B^{o}} \rightarrow B^{o}, & \left(\mathcal{C}_{B^{\prime o}}^{\prime} \rightarrow B^{\prime o}\right)=\left(\mathcal{C}_{\Delta^{o}}^{\prime} \rightarrow \Delta^{o}\right), & \widehat{\mathcal{C}_{\Sigma^{o}}} \rightarrow \Sigma^{o}, \\
\left\{\Lambda_{B}^{\bullet}:=\Lambda_{B^{o}}^{\bullet}\right\}_{\bullet=0}^{10}, & \left\{\Lambda_{\Delta}^{\bullet}:=\Lambda_{B^{\prime o}}^{\bullet}\right\}_{\bullet=0}^{4}, & \left\{\Lambda_{\Sigma}^{\bullet}:=\Lambda_{\Sigma^{o}}^{\bullet}\right\}_{\bullet=0}^{8},
\end{array}
$$

where we have dropped some decorations. We also have the following epimorphisms of groups schemes:

$$
P_{\mid B^{o}} \stackrel{=}{\longrightarrow} \operatorname{Pic}_{\mathcal{C}_{B^{o}} / B^{o}}^{0} \quad P_{\mid B^{\prime o}}^{\prime} \stackrel{=}{\longrightarrow} \operatorname{Pic}_{\mathcal{C}_{\Delta^{\prime}} / \Delta^{o}}^{0}, \quad P_{\mid \Sigma^{o}} \longrightarrow \mathrm{Pic}_{\widehat{\mathcal{C}_{\Sigma^{o}}} / \Sigma^{o}}^{0},
$$

that fiber-by-fiber realize the Chevalley devissages into affine and Abelian parts of the fibers of the corresponding group schemes $P, P^{\prime}$, and $P$ again, respectively

Recall that $\mathscr{L}$ in $\S 4.4$ is a local system on $\Sigma \backslash \Delta$, and that this latter contains $\Sigma^{o}$. Let $\mathscr{L}^{o}:=\mathscr{L}_{\mid \Sigma^{o}}$. Then we have:

$$
i_{\Sigma^{o} *} \mathscr{L}^{o}=i_{\Sigma \backslash \Delta_{*}} \mathscr{L}=i_{\Sigma \backslash \Delta_{!}} \mathscr{L}
$$

where the last equality, is due to the fact that the local monodromy of $\mathscr{L}$ around the diagonal is given by multiplication by -1 .

We introduce the following complexes, viewable in $D^{b} M H M_{a l g}(-)$ or in $D^{b}(-, \mathbb{Q})$, and we simply call them strings -we reserve the term Ngô strings for those strings that actually 
appear in the Decomposition Theorems 7.0.3 and 7.0.4-:

$$
\begin{array}{ll}
\mathscr{S}_{B}:=\bigoplus_{b=0}^{10} \mathscr{I} \mathscr{C}_{B}\left(\Lambda_{B}^{b}\right)[-b], & \mathscr{S}_{\Delta}:=\bigoplus_{b=0}^{4} \mathscr{I} \mathscr{C}_{\Delta}\left(\Lambda_{\Delta}^{b}\right)[-b], \\
\mathscr{S}_{\Sigma}^{+}:=\bigoplus_{b=0}^{8} \mathscr{I} \mathscr{C}_{\Sigma}\left(\Lambda_{\Sigma}^{b}\right)[-b], & \mathscr{S}_{\Sigma}^{-}:=\bigoplus_{b=0}^{8} \mathscr{I} \mathscr{C}_{\Sigma}\left(\Lambda_{\Sigma}^{b} \otimes \mathscr{L}^{o}\right)[-b],
\end{array}
$$

where $\mathscr{L}$ is described in Proposition 4.4.3. They are all subject to the Relative Hard Lefschetz theorem, which here boils down to:

$$
\begin{aligned}
& \Lambda_{B}^{5-\bullet} \simeq \Lambda_{B}^{5+\bullet}(\bullet), \quad \Lambda_{\Delta}^{2-\bullet} \simeq \Lambda_{\Delta}^{2+\bullet}(\bullet), \\
& \Lambda_{\Sigma}^{4-\bullet} \simeq \Lambda_{\Sigma}^{4+\bullet}(\bullet), \quad \Lambda_{\Sigma}^{4-\bullet} \otimes \mathscr{L}^{o} \simeq \Lambda_{\Sigma}^{4+\bullet} \otimes \mathscr{L}^{o}(\bullet)
\end{aligned}
$$

Remark 5.1.1. In the sequel of the paper, we show that the first two and last two summand in each of the strings in (60) are, when viewed in $D^{b}(B, \mathbb{Q})$, shifted sheaves; see (70), (72) and (78).

Remark 5.1.2. The local systems of type $\Lambda$ are endowed with the structure of polarizable variations of pure Hodge structures they acquire as direct image sheaves. We have the following relations between rational graded polarizable pure Hodge structures:

(1) $H^{*}(N)=H^{*}\left(S^{[5]}\right)$; see Lemma 3.2.7.

(2) $H^{*}\left(M^{\prime}\right)=H^{*}\left(S^{[2]}\right)=H^{*}\left(\mathscr{S}_{\Delta}\right)$; see Lemmata 3.2.7 and 5.4.1.

(3) $H^{*}\left(\operatorname{Sym}^{2} M^{\prime}\right)=H^{*}\left(\mathscr{S}_{\Sigma}^{+}\right)$; see Proposition 5.6.1.

(4) $H^{*}\left(M^{\prime 2}\right)=H^{*}\left(\mathscr{S}_{\Delta}\right)^{\otimes 2}=H^{*}\left(\mathscr{S}_{\Sigma}^{+}\right) \oplus H^{*}\left(\mathscr{S}_{\Sigma}^{-}\right)$; see Lemma 5.5.1 and Proposition 5.6.1.

(5) By the forthcoming Proposition 5.7.2, and by Lemma 4.1.3, we have:

$$
H^{*}(\widetilde{M}) \supseteq I H^{*}(M) \supseteq H^{*}\left(\mathscr{S}_{B}\right) \subseteq H^{*}(N) .
$$

5.2. The Decomposition Theorem for the genus two universal curve $\mathcal{C}^{\prime} \rightarrow B^{\prime}$.

The complete linear system $B^{\prime}=|H| \simeq \mathbb{P}^{2}$ of our genus two curve $C$ on $K 3$ is two dimensional and we have the universal curve morphism:

$$
\gamma^{\prime}: \mathcal{C}^{\prime 3} \rightarrow B^{\prime 2}=|C|
$$

The total space $\mathcal{C}^{\prime}$ is nonsingular and three dimensional. The fibers of $\gamma^{\prime}$ are all integral curves. Let $B^{\prime o} \subseteq B^{\prime}$ be the open subset of regular values of $\gamma^{\prime}$, and let $i_{B^{\prime o}}: B^{\prime o} \rightarrow B^{\prime}$ be the resulting open embedding. Let $R_{\mathcal{C}^{\prime}}^{1}$ be local system restriction of $R^{1} \gamma_{*}^{\prime} \mathbb{Q}$ to $B^{\prime o}$; it coincides with $\Lambda_{\Delta}^{1}:=\Lambda_{B^{\prime}}^{1}:=\Lambda_{B^{\prime o}}^{1}$ in (57).

Lemma 5.2.1. (Decomposition Theorem for $R \gamma_{*}^{\prime} \mathbb{Q}_{\mathcal{C}^{\prime}}$ ) The Decomposition Theorem for the direct image complex $R \gamma_{*}^{\prime} \mathbb{Q}$ takes the following form:

$$
R \gamma_{*}^{\prime} \mathbb{Q}_{\mathcal{C}^{\prime}} \simeq \mathbb{Q}_{B^{\prime}} \oplus i_{*} \Lambda_{\Delta}^{1}[-1] \oplus \mathbb{Q}_{B^{\prime}}[-2](-1) .
$$

Proof. For reasons of bookkeeping, we find it more natural to prove the equivalent statement:

$$
R \gamma_{*}^{\prime} \mathbb{Q}_{\mathcal{C}^{\prime}}[3] \simeq \mathbb{Q}_{B^{\prime}}[2][1] \oplus i_{*} \Lambda_{\Delta}^{1}[2][0] \oplus \mathbb{Q}_{B^{\prime}}[2][-1](-1)
$$


where the summands appear with increasing perverse degree $-1,0,1$.

The following is standard (cf. [de-PCMI, Theorem 1.5.3 and Fact 4.5.4]: the following summands appear in the Decomposition Theorem for $R \gamma_{*}^{\prime} \mathbb{Q}_{\mathcal{C}^{\prime}}[3]$ :

$$
\mathbb{Q}_{B^{\prime}}[2][1] \oplus I C_{B^{\prime}}\left(\Lambda_{\Delta}^{1}\right)[0] \oplus \mathbb{Q}_{B^{\prime}}[2][-1](-1) .
$$

Since the fibers of $\gamma^{\prime}$ are irreducible, we have that $R^{2} \gamma_{*}^{\prime} \mathbb{Q}=\mathbb{Q}_{B^{\prime}}$, hence:

$$
I C_{B^{\prime}}\left(\Lambda_{B^{\prime}}^{1}\right)[0]=i_{B^{\prime o} *} \Lambda_{B^{\prime}}^{1}[2] .
$$

If in (65) there were other summands than these three above, then, by the Relative Hard Lefschetz symmetries, they would need to be in perverse degree zero: else, they would contribute nontrivially to $R^{j} \gamma_{*}^{\prime} \mathbb{Q}=0$ for some $j \geq 3$, a contradiction (the fibers of $\gamma$ are curves).

If there were other summands in perverse degree zero, then they would be of the form $I^{0} \oplus I^{1}$, where the exponent refers to the dimension of the support of the intersection complex. An $I^{0}$ would contribute non trivially to $R^{3} \gamma_{*}^{\prime} \mathbb{Q}=0$, a contradiction. An $I^{1}$ would be a sheaf placed in cohomological degree -1 , and it would thus contribute non trivially to $R^{2} \gamma_{*}^{\prime} \mathbb{Q}$. Since we know that this latter is $\mathbb{Q}_{B^{\prime}}$ and is already accounted for by the summand $\mathbb{Q}_{B^{\prime}}[2][-1](-1)$ on the rhs of (65), we get a contradiction.

\subsection{The Decomposition Theorem for the genus five universal curve $\mathcal{C} \rightarrow B$.}

The complete linear system $|2 C|$ is five dimensional so that we have the associated universal family of curves:

$$
\gamma: \mathcal{C}^{6} \rightarrow B^{5}=|2 C|
$$

The total space $\mathcal{C}$ is nonsingular and six dimensional. The fibers of $\gamma$ are integral curves away from the integral divisor $\Sigma:=\operatorname{Sym}^{2} B^{\prime} \subseteq B$. Let $\Delta \subseteq \Sigma$ be the diagonal. The fibers of $\gamma$ over $\Sigma \backslash \Delta$ are reduced and are the union of two irreducible components. The two irreducible components of a fiber over the locus $\Sigma^{o}:=\operatorname{Sym}^{2} B^{\prime o} \backslash \Delta$ are exchanged by the monodromy action around the diagonal. Let $\mathscr{L}$ be the evident rank one local system on $\Sigma \backslash \Delta$ with monodromy -1 around $\Delta$ (cf. §4.4). We view it as a polarizable variation of pure Hodge structures of Hodge-Tate type, and of weight zero on $\Sigma \backslash \Delta$. We have:

$$
\left(R^{2} \gamma_{*} \mathbb{Q}_{\mathcal{C}}\right)_{\mid \Sigma \backslash \Delta}=\left(\mathbb{Q}_{\Sigma \backslash \Delta} \oplus \mathscr{L}\right)(-1) .
$$

The fibers of $\gamma$ over $\Delta$ are irreducible non-reduced: they are two times a curve in $|C|$ (recall that on our $K 3$ surface $S$ all curves in $|C|$ are integral).

Let $B^{o} \subseteq B$ be the open subset of regular values of $\gamma$. Let $R_{\mathcal{C}}^{1}$ be the local system restriction of $R^{1} \gamma_{*} \mathbb{Q}$ to $B^{o}$; it coincides with $\Lambda_{B}^{1}$ in (57). Let $i_{B^{o}}: B^{o} \rightarrow B$ be the evident open immersion.

Lemma 5.3.1. (Decomposition Theorem for $R \gamma_{*} \mathbb{Q}_{\mathcal{C}}$ ) The Decomposition Theorem for $R \gamma_{*} \mathbb{Q}$ takes the following form:

$$
R \gamma_{*} \mathbb{Q}_{\mathcal{C}} \simeq \mathbb{Q}_{B} \oplus\left(i_{B^{0} *} \Lambda_{B}^{1}[-1] \oplus i_{\Sigma \backslash \Delta_{*}} \mathscr{L}[-2](-1)\right) \oplus \mathbb{Q}_{B}[-2](-1) .
$$

Proof. We prove the equivalent:

$$
R \gamma_{*} \mathbb{Q}_{\mathcal{C}}[6] \simeq \mathbb{Q}_{B}[5][1] \oplus\left(i_{B^{0} *} \Lambda_{B}^{1}[5] \oplus i_{\Sigma \backslash \Delta_{*}} \mathscr{L}[4](-1)\right)[0] \oplus \mathbb{Q}_{B}[5][-1](-1),
$$


where the summands are grouped by perversity $(-1,0,1)$.

By looking at the regular part of $\gamma$, we deduce that the following summands appear in the Decomposition Theorem for $R \gamma_{*} \mathbb{Q}_{\mathcal{C}}[6]$ :

$$
\mathbb{Q}_{B}[5][1] \oplus I C_{B}\left(\Lambda_{B}^{1}\right)[0] \oplus \mathbb{Q}_{B}[5][-1](-1) .
$$

By the Relative Hard Lefschetz symmetries, any additional summand would need to be in perverse degree zero: else, they would contribute nontrivially to $R^{j} \gamma_{*} \mathbb{Q}=0$ for some $j \geq 3$, a contradiction (the fibers of $\gamma$ are curves).

The direct summand contribution of $I C_{B}\left(\Lambda_{B}^{1}\right)[0]$ to $R^{2} \gamma_{*} \mathbb{Q}$ is the sheaf $\mathcal{H}^{-4}\left(I C_{B}\left(\Lambda_{B^{\circ}}^{1}\right)\right)$. By the condition of support for intersection complexes, this sheaf is supported in dimension $\leq 3$. Recall that the curves over the points of the four dimensional $\Sigma^{o}$ have two irreducible components. It follows that there must be a contribution of $\Sigma$, to the l.h.s. of (69), of the form $I C_{\Sigma}(\mathfrak{L})[0]$, where $\mathfrak{L}$ is some rank one local system on some open dense subset $\Sigma^{\prime}$ of $\Sigma$. Since it contributes to $R^{2} \gamma_{*} \mathbb{Q}$, as a polarizable variation of pure Hodge structures, the local systems $\mathfrak{L}$ is necessarily of pure Hodge-Tate type with weight 2 . The intersection complex $I C_{\Sigma}(\mathfrak{L})$ has non zero cohomology sheaves in degrees contained in the interval $[-4,-1]$, where $i_{\Sigma^{\prime}}: \Sigma \rightarrow B$ is the evident locally closed embedding. The sheaf $\mathcal{H}^{-4}\left(I C_{\Sigma}(\mathfrak{L})\right)$ contributes to $R^{2} \gamma_{*} \mathbb{Q}$. The remaining cohomology sheaves in degrees in the interval $[-3,-1]$ contribute to $R^{j} \gamma_{*} \mathbb{Q}$ for $j \geq 3$, and are thus zero. It follows that $I C_{\Sigma}(\mathfrak{L})$ is the sheaf $i_{\Sigma^{\prime} *} \mathfrak{L}$ placed in degree -4 . The local system $\mathfrak{L}$ agrees with $\mathscr{L}$ where it is defined. Since the two local systems are both of rank one and of Hodge-Tate type, it follows that $\mathfrak{L}=\mathscr{L}(-1)$, where they are both defined. It follows that $i_{\Sigma^{\prime} *} \mathfrak{L}=i_{\Sigma \backslash \Delta_{*}} \mathscr{L}(-1)$, so that $I C_{\Sigma}(\mathfrak{L})=i_{\Sigma \backslash \Delta_{*}} \mathscr{L}[4](-1)$.

Note that the stalks of $R^{2} \gamma_{*} \mathbb{Q}$ on $\Delta$ are one dimensional. Note also that $i_{\Sigma \backslash \Delta_{*}} L=$ $i_{\Sigma \backslash \Delta_{1}} L$ has zero stalks on $\Delta$ (no local invariants near the diagonal).

By inspecting the cohomology sheaves of $I C_{B}\left(\Lambda_{B}^{1}\right)$, we deduce that this perverse sheaf is a sheaf placed in degree -5 , hence of the predicated form:

$$
\left.I C_{B}\left(\Lambda_{B}^{1}\right)=i_{B^{o} *} \Lambda_{B^{o}}^{1}[4] \quad \text { (equivalently, } \mathscr{I}_{\mathscr{C}_{B}}\left(\Lambda_{B}^{1}\right)=i_{B^{o} *} \Lambda_{B}^{1}\right) .
$$

\subsection{The Ngô strings for the Lagrangian fibration $p: M^{\prime} \rightarrow B^{\prime}$.}

Recall that $M^{\prime}$ is a four-dimensional moduli space which admits the Lagrangian fibration $p: M^{\prime} \rightarrow B^{\prime}(19)$, that we have the local systems of type $\Lambda$ (19), the strings (60) and the isomorphisms (61).

Lemma 5.4.1. (Ngô strings for $p: M^{\prime} \rightarrow B^{\prime}$ ) The Decomposition Theorem for $p$ : $M^{\prime} \rightarrow B^{\prime}$ has the following form:

$$
R p_{*} \mathbb{Q}_{M^{\prime}} \simeq \mathscr{S}_{\Delta}=\mathbb{Q}_{B^{\prime}} \bigoplus \bigoplus_{b=1}^{3} \mathscr{I}_{C_{B^{\prime}}}\left(\Lambda_{\Delta}^{b}\right)[-b] \bigoplus \mathbb{Q}_{B^{\prime}}[-4](-2) .
$$

where, moreover, the following intersection cohomology complex is a sheaf:

$$
\mathscr{I} \mathscr{C}_{B^{\prime}}\left(\Lambda_{\Delta}^{2 \pm 1}\right)=i_{B^{\prime o} *} R_{\mathcal{C}^{\prime}}^{1}\left(-\frac{1}{2} \mp \frac{1}{2}\right)
$$


Proof. By Proposition 3.4.6, the morphism $p: M^{\prime} \rightarrow B^{\prime}$ is part of a $\delta$-regular weak Abelian fibration. We can thus apply Theorem 7.0.3, so that, in view of the fact that the fibers of $p$ are irreducible (they are the compactified Jacobians of the integral locally planar curves in $B^{\prime}$ [Re-1980]), we have that the Decomposition Theorem has the desired form (71).

Since the general fiber of the group scheme $\mathrm{Pic}_{\mathcal{C}^{\prime} / B^{\prime}}^{0}$ is the Jacobian of a nonsingular curve of genus two, by (61) $\Lambda_{\Delta}^{1} \simeq \Lambda_{\Delta}^{3}(1)$, so that in order to prove (72) it is enough to prove that:

$$
\mathscr{I}_{B_{B^{\prime}}}\left(\Lambda_{\Delta}^{1}\right)=i_{B^{\prime o} *}\left(\left(R^{1} \gamma_{*}^{\prime} \mathbb{Q}_{\mathcal{C}^{\prime}}\right)_{\mid B^{\prime o}}\right)
$$

which follows from (66) and the fact, already observed at the beginning of $\S 5.2$, that $\Lambda_{\Delta}^{1}=\left(R^{1} \gamma_{*}^{\prime} \mathbb{Q}_{\mathcal{C}^{\prime}}\right)_{\mid B^{\prime o}}$.

5.5. The Ngô strings for the Lagrangian fibration $p^{2}: M^{\prime} \times M^{\prime} \rightarrow B^{\prime} \times B^{\prime}$.

Let $M^{\prime}$ be as in the beginning of $\S 5.4$. Given a product with the projections onto the factors, the box product is the ordinary tensor product of the pull-backs via the projections. We consider the Lagrangian fibration $p^{2}=p \times p: M^{\prime} \times M^{\prime} \rightarrow B^{\prime} \times B^{\prime}$. Recall (19) that for simplicity, we are denoting $\Lambda_{B^{\prime o}}^{\bullet}$ simply by $\Lambda_{\Delta}^{\bullet}$. We denote by $\Lambda_{\Delta \times \Delta}^{\bullet}$ the analogous local systems on $B^{\prime o} \times B^{\prime o}$ associated with $P^{\prime} \times P^{\prime} \rightarrow B^{\prime} \times B^{\prime}$.

Lemma 5.5.1. The Decomposition Theorem for $p^{2}$ has the following form:

$$
\begin{aligned}
R p_{*}^{2} \mathbb{Q}_{M^{\prime} \times M^{\prime}} & \simeq \mathscr{S}_{\Delta} \otimes \mathscr{S}_{\Delta} \\
& =\bigoplus_{b=0}^{8} \bigoplus_{b^{\prime}+b^{\prime \prime}=b}\left(\mathscr{I}_{C_{B^{\prime}}}\left(\Lambda_{\Delta}^{b^{\prime}}\right) \otimes \mathscr{I}_{C_{B^{\prime}}}\left(\Lambda_{\Delta}^{b^{\prime \prime}}\right)\right)[-b] \\
& =\bigoplus_{b=0}^{8} \mathscr{I}_{C_{B^{\prime} \times B^{\prime}}}\left(\Lambda_{\Delta \times \Delta}^{b}\right)[-b] .
\end{aligned}
$$

Moreover, the following summands are sheaves:

$$
\begin{gathered}
\mathscr{I} \mathscr{C}_{B^{\prime} \times B^{\prime}}\left(\Lambda_{\Delta \times \Delta}^{4 \pm 4}\right)(2 \pm 2)=\mathbb{Q}_{B^{\prime} \times B^{\prime}} \\
\mathscr{I} \mathscr{C}_{B^{\prime} \times B^{\prime}}\left(\Lambda_{\Delta \times \Delta}^{4 \pm 3}\right)=\bigoplus_{j=1}^{2} \operatorname{pr}_{j}^{*} \mathscr{I} \mathscr{C}_{B^{\prime}}\left(\Lambda_{\Delta}^{1}\right)(3 \pm 3)=\bigoplus_{j=1}^{2} \operatorname{pr}_{j}^{*} i_{B^{\prime o}{ }_{*}} \Lambda_{B^{\prime o}}^{1}(3 \pm 3) .
\end{gathered}
$$

Proof. For reasons of bookkeeping, we prove the theorem for the shifted $R p_{*}^{2} \mathbb{Q}_{M^{\prime} \times M^{\prime}}[8]$.

The projections $\operatorname{pr}_{j}: B^{\prime} \times B^{\prime}$ are smooth of relative dimension 2, so that the functors $\operatorname{pr}_{j}^{*}[2]$ preserve pure Hodge complexes and intersection complexes (the coefficients are the pulled-back coefficients). The desired conclusions (73) follows by: taking Lemma 5.4.1; applying the Künneth formula keeping track of the perversities; organizing the summands. The identities (74) follow from the isomorphisms $\Lambda_{\Delta \times \Delta}^{0} \simeq \Lambda_{\Delta \times \Delta}^{8}(4) \simeq \mathbb{Q}_{B^{\prime \circ} \times B^{\prime o}}$ (use (61) and the irreducibility of the fibers) and the fact that $B^{\prime} \times B^{\prime}$ is nonsingular. The identities (75) follow from the natural isomorphism (61) $\Lambda_{\Delta \times \Delta}^{1} \simeq \Lambda_{\Delta \times \Delta}^{7}$ (3) (the general fiber of the group scheme is the product of the Jacobians of two nonsingular projective curves of genus two), as well as (66), with the shift changed from [2] to [4] to take into account that we are pulling back along smooth morphisms, the projections, of relative dimension 2 . 
5.6. The Ngô strings for the Lagrangian fibration $p^{(2)}: \mathrm{Sym}^{2} M^{\prime} \rightarrow \mathrm{Sym}^{2} B^{\prime}$.

The morphism $M^{\prime 2}: M^{\prime 2} \rightarrow B^{\prime 2}$ is equivariant for the action of the symmetric group in two letters, so that it induces the natural morphism $p^{(2)}: \mathrm{Sym}^{2} M^{\prime} \rightarrow \mathrm{Sym}^{2} B^{\prime}$ on the two-fold symmetric products. We have the commutative diagram:

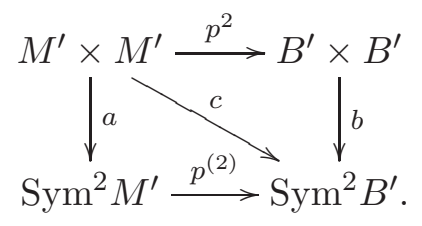

Recall that we have the the local systems $\Lambda_{\Sigma}^{\bullet}$ (57) and $\mathscr{L}^{\circ}$ (59) defined on $\Sigma^{o}=$ $\operatorname{Sym}^{2} B^{\prime o} \backslash \Delta_{B^{\prime}}$ and the strings $\mathscr{S}_{\Sigma}^{ \pm}$defined in (60).

Proposition 5.6.1. The Decomposition Theorems for the morphisms $c$ and $p^{(2)}$ take the following form:

$$
R c_{*} \mathbb{Q}_{M^{\prime} \times M^{\prime}}=\mathscr{S}_{\Sigma}^{+} \oplus \mathscr{S}_{\Sigma}^{-} ; \quad R p_{*}^{(2)} \mathbb{Q}_{\mathrm{Sym}^{2} M^{\prime}} \simeq \mathscr{S}_{\Sigma}^{+}
$$

Moreover, we have that the following terms are sheaves:

$$
\begin{aligned}
\mathscr{I} \mathscr{C}_{\Sigma}\left(\Lambda_{\Sigma}^{4 \pm 4}\right) & =\mathbb{Q}_{\Sigma}(2 \pm 2), & \mathscr{I} \mathscr{C}_{\Sigma}\left(\Lambda_{\Sigma}^{4 \pm 4} \otimes \mathscr{L}^{o}\right) & =i_{\Sigma^{o} *} \mathscr{L}_{B^{\prime}}(2 \pm 2)=i_{\Sigma^{o} !} \mathscr{L}_{B^{\prime}}(2 \pm 2), \\
\mathscr{I} \mathscr{C}_{\Sigma}\left(\Lambda_{\Sigma}^{4 \pm 3}\right) & =i_{\Sigma^{o} *} \Lambda_{\Sigma}^{4 \pm 3}(3 \pm 3), & \mathscr{I} \mathscr{C}_{\Sigma}\left(\Lambda_{\Sigma}^{4 \pm 3} \otimes \mathscr{L}^{o}\right) & =i_{\Sigma^{o} *}\left(\Lambda_{\Sigma}^{4 \pm 3} \otimes \mathscr{L}^{o}\right)(3 \pm 3) .
\end{aligned}
$$

Proof. Denote by $\mathscr{L}_{M^{\prime}}$ the rank one local system on $\mathrm{Sym}^{2} M^{\prime} \backslash \Delta_{B^{\prime}}$ with monodromy -1 around the diagonal; it is pure of Hodge-Tate type, with weight zero. We have $R a_{*} \mathbb{Q}_{M^{\prime} \times M^{\prime}}=a_{*} \mathbb{Q}_{M^{\prime} \times M^{\prime}}=\mathbb{Q}_{\mathrm{Sym}^{2} M^{\prime}} \oplus\left(i_{\mathrm{Sym}^{2} M^{\prime} \backslash \Delta_{M^{\prime}}}\right)_{*} \mathscr{L}_{M^{\prime}}$. It follows that:

$$
R c_{*} \mathbb{Q}_{M^{\prime} \times M^{\prime}}=R p_{*}^{(2)} \mathbb{Q}_{\mathrm{Sym}^{2} M^{\prime}} \oplus R p_{*}^{(2)} i_{\mathrm{Sym}^{2} M^{\prime} \backslash \Delta_{M^{\prime} *}} \mathscr{L}_{M^{\prime}}
$$

By the functoriality of the direct image, the fact that $b$ is finite and (73), we have $R c_{*} \mathbb{Q}_{M^{\prime} \times M^{\prime}} \simeq R b_{*} R p_{*}^{2} \mathbb{Q}_{M^{\prime} \times M^{\prime}}=b_{*}\left(\mathscr{S}_{\Delta} \otimes \mathscr{S}_{\Delta}\right)$. Note that $\mathscr{S}_{\Delta}$, being the direct sum of shifted intersection complexes supported exactly on $B^{\prime}$, is determined, via the intermediate extension functor, by its restriction to any dense open subset of $B^{\prime}$. The analogous fact remains true for $\mathscr{S}_{\Delta} \otimes \mathscr{S}_{\Delta}$ on $B^{\prime} \times B^{\prime}$. It follows that the analogous fact remains true for $b_{*}\left(\mathscr{S}_{\Delta} \otimes \mathscr{S}_{\Delta}\right) \simeq R p_{*}^{(2)} \mathbb{Q}_{\mathrm{Sym}^{2} M^{\prime}} \oplus R p_{*}^{(2)} i_{\mathrm{Sym}^{2} M^{\prime} \backslash \Delta_{M^{\prime}}} \mathscr{L}_{M^{\prime}}$ on Sym ${ }^{2} B^{\prime}$ : in fact, the morphism $b$ being finite and surjective, the direct image $b_{*}$ sends intersection complexes supported exactly on $B^{\prime 2}$ to ones supported exactly on $\mathrm{Sym}^{2} B^{\prime}$.

It is thus enough to verify the desired assertions (77) concerning (79) on any Zariskidense open subset $U$ of $\mathrm{Sym}^{2} B^{\prime}$. On any such open subset $U,(79)$ takes the form:

$$
\left(R p_{*}^{(2)} \mathbb{Q}_{\mathrm{Sym}^{2} M^{\prime}}\right)_{\mid U} \oplus\left(R p_{*}^{(2)} i_{\mathrm{Sym}^{2} M^{\prime} \backslash \Delta_{M^{\prime} *}} \mathscr{L}_{M^{\prime}}\right)_{\mid U}
$$

We set $U:=\Sigma^{o}:=\operatorname{Sym}^{2} B^{\prime o} \backslash \Delta_{B^{\prime o}}$. In view of Remark 4.4.4.(3), recalling that $\mathscr{L}$ is defined on $\operatorname{Sym}^{2} B^{\prime} \backslash \Delta_{B^{\prime}}$, and that $\mathscr{L}_{B^{\prime}}=p^{(2)^{*}} \mathscr{L}$, and by using the projection formula, we have that $(80)$ reads as follows:

$$
\left(R p_{*}^{(2)} \mathbb{Q}_{\mathrm{Sym}^{2} M^{\prime}}\right)_{\mid \Sigma^{o}} \oplus\left(\left(R p_{*}^{(2)} \mathbb{Q}_{\mathrm{Sym}^{2} M^{\prime}}\right)_{\mid \Sigma^{o}} \otimes \mathscr{L}_{\mid \Sigma^{\circ}}\right) .
$$


The proper morphism $p^{(2)}$ is smooth over $\Sigma^{o}$, so that, by Deligne's theorem [de-PCMI, Theorem 1.5.3], i.e. the Decomposition Theorem for proper smooth morphisms, we have that:

$$
\left(R p_{*}^{(2)} \mathbb{Q}_{\mathrm{Sym}^{2} M^{\prime}}\right)_{\mid \Sigma^{o}} \simeq \bigoplus_{\bullet=0}^{8}\left(R^{\bullet} p_{*}^{(2)} \mathbb{Q}_{\mathrm{Sym}^{2} M^{\prime}}\right)_{\mid \Sigma^{o}}[-\bullet]
$$

Now, the rhs of (82) coincides with $\mathscr{S}_{\Sigma \mid U}^{+}$: this follows from the fact that the morphism $\left(p^{(2)}\right)_{\mid \Sigma^{o}}$ is a torsor for the group scheme $(58)\left(\mathrm{Pic}_{\overline{\mathcal{C}}_{\Sigma^{o}} / \Sigma^{o}}^{0}\right)$. This can also be seen to follow directly from the Ngô String Theorem 7.0.3.

We have thus proved (77).

All the summands in $\mathscr{S}_{\Sigma}^{+}$and $\mathscr{S}_{\Sigma}^{-}$have been shown to be direct summands of terms of the form $b_{*} \mathscr{I} \mathscr{C}_{B^{\prime o} \times B^{\prime o}}\left(\Lambda_{\Delta \times \Delta}^{\bullet}\right)$. Since the direct image functor $b_{*}$ sends sheaves to sheaves, in view of (73) and (75), the intersection complexes in (78) are shifted sheaves, and thus have the predicated form.

\subsection{Ngô strings for the Lagrangian fibrations $\widetilde{M}, N \rightarrow B$.}

This section is devoted to proving Proposition 5.7.2. While it falls falls short of establishing the exact shape of the Decomposition Theorem for the complexes $R \widetilde{m}_{*} \mathbb{Q}_{\widetilde{M}}$ and $R n_{*} \mathbb{Q}_{N}$, the fact that this shortcoming is measured by the same integer $\epsilon$ in both expressions (87), is key to proving our main Theorem A in $\S 6.1$.

Before embarking in the proof of Proposition 5.7.2, we list some facts we need.

\section{Fact 5.7.1.}

(1) By the very definition (60), the cohomology sheaf in degree 10 of the string $\mathscr{S}_{B}$ takes the form: $\mathcal{H}^{10}\left(\mathscr{S}_{B}\right)=\mathbb{Q}_{B} \oplus \oplus_{i=1}^{4} \mathcal{H}^{i}\left(\mathscr{I}_{\mathscr{C}_{B}}\left(\Lambda_{B^{o}}^{10-i}\right)\right)$, where the direct sum ends at 4 by the conditions of support for intersection complexes. These same conditions tell us that $\operatorname{dim} \operatorname{support} \mathcal{H}^{i}\left(\mathscr{I}_{\mathscr{C}_{B}}\left(\Lambda_{B^{o}}^{10-i}\right)\right) \leq 4-i$, for $1 \leq i \leq 4$. The Relative Hard Lefschetz isomorphism $\Lambda_{B^{o}}^{1}=\Lambda_{B^{o}}^{9}(4)$, combined with Lemma 5.3.1 (cf. (70)), implies that $\mathcal{H}^{1}\left(\mathscr{I}_{\mathscr{C}}{ }_{B}\left(\Lambda_{B^{\circ}}^{9}\right)\right)=0$. In summary, we have:

$$
\mathcal{H}^{10}\left(\mathscr{S}_{B}\right)=\mathbb{Q}_{B} \oplus{ }^{\leq 2} \mathcal{H}^{2}\left(\mathscr{I} \mathscr{C}_{B}\left(\Lambda_{B^{o}}^{8}\right)\right) \oplus{ }^{\leq 1} \mathcal{H}^{3}\left(\mathscr{I} \mathscr{C}_{B}\left(\Lambda_{B^{o}}^{7}\right)\right) \oplus \leq 0 \mathcal{H}^{4}\left(\mathscr{I} \mathscr{C}_{B}\left(\Lambda_{B^{o}}^{6}\right)\right),
$$

where the exponents on the left are the upper bounds on the dimensions of the respective supports.

(2) In the same vein, but by using Proposition 5.6.1 in place of Lemma 5.3.1, we have that the cohomology sheaf in degree 8 of the string $\mathscr{S}_{\Sigma}^{+}$-i.e. in degree 0 for $S_{\Sigma}^{+}$ takes the form:

$$
\mathcal{H}^{8}\left(\mathscr{S}_{\Sigma}^{+}\right)=\mathbb{Q}_{\Sigma} \oplus \leq{ }^{\leq 1} \mathcal{H}^{2}\left(\mathscr{I}_{\mathscr{C}_{\Sigma}}\left(\Lambda_{\Sigma^{o}}^{6}\right)\right) \oplus{ }^{\leq 0} \mathcal{H}^{3}\left(\mathscr{I}_{\mathscr{C}_{B}}\left(\Lambda_{\Sigma^{o}}^{5}\right)\right) .
$$

where the exponents on the left are the upper bounds on the dimensions of the respective supports. This is improved in (91).

(3) Similarly, the cohomology sheaf in degree 8 of the string $\mathscr{S}_{\Sigma}^{-}$takes the form:

$$
\mathcal{H}^{8}\left(\mathscr{S}_{\Sigma}^{-}\right)=i_{\Sigma \backslash \Delta_{*}} \mathscr{L} \oplus \leq{ }^{1} \mathcal{H}^{2}\left(\mathscr{I} \mathscr{C}_{\Sigma}\left(\Lambda_{\Sigma^{o}}^{6} \otimes \mathscr{L}^{o}\right)\right) \oplus \leq 0 \mathcal{H}^{3}\left(\mathscr{I}_{\mathscr{C}_{B}}\left(\Lambda_{\Sigma^{o}}^{5} \otimes \mathscr{L}^{o}\right)\right) \text {, }
$$

where the exponents on the left are the upper bounds on the dimensions of the respective supports. This is improved to (92). 
(4) In the same vein, but by using Lemma 5.4.1 in place of Lemma 5.3.1, coupled with the fact, due to the conditions of support, that $\mathcal{H}^{2} \mathscr{I} \mathscr{C}(\mathfrak{L})=0$ for any system of coefficients $\mathfrak{L}$ on a surface, we have that the cohomology sheaf in degree 4 of the string $\mathscr{S}_{\Delta}$ reads as follows:

$$
\mathcal{H}^{4}\left(\mathscr{S}_{\Delta}\right)=\mathbb{Q}_{\Delta}
$$

(5) The following is clear: if $T$ is an irreducible variety and $F \simeq \mathbb{Q}_{T}^{\oplus r}$ is a constant sheaf on $T$, then $F$ does not admit a direct summand supported on any proper subvariety.

(6) The local system $\mathscr{L}$ on $\Sigma \backslash \Delta$ of Proposition 4.4 .3 satisfies the following properties Fact 4.4.4 (cf. the proof of Proposition 5.3.1): (i) $i_{\Sigma \backslash \Delta_{*}} \mathscr{L}=i_{\Sigma \backslash \Delta_{1}} \mathscr{L}$ (because there are no local monodromy invariants around the diagonal); (II) for every Zariski dense open subset $j: U \subseteq \Sigma \backslash \Delta$, we have that $\mathscr{L}=j_{*} j^{*} \mathscr{L}$ (true for any local system, due to the normality of the varieties involved); (IIi) $i_{U *}\left(\mathscr{L}_{\mid U}\right)=i_{\Sigma \backslash \Delta_{*}} \mathscr{L}$ on $B$ (follows immediately from (II) by functoriality).

Proposition 5.7.2. The Decomposition Theorem for $R \widetilde{m}_{*} \mathbb{Q}_{\widetilde{M}}, R n_{*} \mathbb{Q}_{N}$ in $D^{b} M H M_{\text {alg }}(B)$ takes the following form:

$$
\begin{aligned}
& R \widetilde{m}_{*} \mathbb{Q}_{\widetilde{M}} \simeq \mathscr{S}_{B} \bigoplus \mathscr{S}_{\Sigma}^{+}[-2](-1) \bigoplus \mathscr{S}_{\Delta}^{\oplus\left(1+\epsilon_{\widetilde{M}}\right)}[-6](-3), \\
& R n_{*} \mathbb{Q}_{N} \simeq \mathscr{S}_{B} \bigoplus \mathscr{S}_{\Sigma}^{-}[-2](-1) \bigoplus \mathscr{S}_{\Delta}^{\oplus \epsilon_{N}}[-6](-3),
\end{aligned}
$$

where $\epsilon_{\widetilde{M}}=\epsilon_{N}=0$, or 1 .

Proof. By virtue of Proposition 3.4.6 about $\delta$-regularity, we can apply Theorem 7.0.3 on Ngô strings.

We first deal with $R \widetilde{m}_{*} \mathbb{Q}_{\widetilde{M}}$.

By Corollary 4.1.4, the subvarieties $B, \Sigma$ and $\Delta$ are supports; in particular, each one of them must contribute the associated Ngô string as a direct summand of $R \widetilde{m}_{*} \mathbb{Q}_{\widetilde{M}}[10]$.

By Proposition 4.4.3, the local systems of type $L$ appearing in Theorem 7.0.3 applied to each of these three supports are constant of some strictly positive ranks $r_{\widetilde{M}, B}, r_{\widetilde{M}, \Sigma}, r_{\widetilde{M}, \Delta}$, and the same is true after the push-forward (114).

It follows that the direct sum of the Ngô strings associated with these three supports takes the following form:

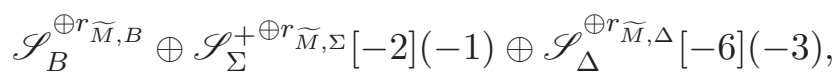

so that, according to Fact 5.7.1(1,2,4), the combined contribution to the direct image $R^{10} \widetilde{m}_{*} \mathbb{Q}_{\widetilde{M}}$ stemming from the local systems of type $L$ for these three Ngô strings takes the form:

$$
\left(\mathbb{Q}_{B}^{\oplus r_{\widetilde{M}, B}} \oplus \mathbb{Q}_{\Sigma}^{\oplus r_{\widetilde{M}, \Sigma}} \oplus \mathbb{Q}_{\Delta}^{\oplus r_{\widetilde{M}, \Delta}}\right)(-5) .
$$

Proposition 4.4.3 implies that $r_{\widetilde{M}, B}=r_{\widetilde{M}, \Sigma}=1$ and that $1 \leq r_{\widetilde{M}, \Delta} \leq 2$, Moreover:

(1) $r_{\widetilde{M}, \Delta}=2$ if an only if the combined contribution of the two Ngô strings $\mathscr{S}_{B}$ and $\mathscr{S}_{\Sigma}^{+}$to $R_{\widetilde{M} \mid \Delta}^{10}$, i.e. the direct sum of the l.h.s. of (83) and (84) restricted to $\Delta$, is exactly $\mathbb{Q}_{\Delta}^{\oplus 2}$; 
(2) $r_{\widetilde{M}, \Delta}=1$ if an only if the above contribution is exactly $\mathbb{Q}_{\Delta}^{\oplus 3}$.

We define $\epsilon_{\widetilde{M}}$ so that $1+\epsilon_{\widetilde{M}}=r_{\widetilde{M}, \Delta}$ :

$$
\epsilon_{\widetilde{M}}:=r_{\widetilde{M}, \Delta}-1 .
$$

The 1.h.s. of (87) has been established.

Before studying $R n_{*} \mathbb{Q}_{N}$, we note in passing that the above analysis, Fact 5.7.1.(1,2,5) and Proposition 4.4.3 imply the following improvements of (83) and of (84):

$$
\mathcal{H}^{10}\left(\mathscr{S}_{B}\right)=\mathbb{Q}_{B} \oplus \mathcal{H}^{2}\left(\mathscr{I} \mathscr{C}_{B}\left(\Lambda_{B^{o}}^{8}\right)\right), \quad \mathcal{H}^{2}\left(\mathscr{I}_{\mathscr{C}_{B}}\left(\Lambda_{B^{o}}^{8}\right)\right) \simeq \mathbb{Q}_{\Delta}^{\oplus \epsilon_{B}^{2,8}}, \quad \mathcal{H}^{8}\left(\mathscr{S}_{\Sigma}^{+}\right)=\mathbb{Q}_{\Sigma},
$$

where $\epsilon_{B}^{2,8}=1-\epsilon_{\widetilde{M}}$. Note that $r_{\widetilde{M}, \Delta}=1,2$, and $r_{\widetilde{M}, \Delta}=2$ iff $\epsilon_{\widetilde{M}}=1$ iff $\epsilon_{B}^{2,8}=0$.

We now study $R n_{*} \mathbb{Q}_{N}$.

By virtue of Proposition 3.4.6 about $\delta$-regularity, we can apply Theorem 7.0.3 on Ngô strings. By Proposition 4.4.3, we have that $R_{N \mid B \backslash \Sigma}^{10} \simeq \mathbb{Q}_{B \backslash \Sigma}(-5)$, which, jointly with Corollary 7.0.3 on the shape of Ngô strings and the associated local system contributing to $R_{\mid B \backslash \Sigma}^{10}$, implies that $\left(R n_{*} \mathbb{Q}_{N}\right)_{\mid B \backslash \Sigma} \simeq\left(\mathscr{S}_{B}\right)_{\mid B \backslash \Sigma}$. It follows that the Ngô string for $R n_{*} \mathbb{Q}_{N}$ associated with $B$ is $\mathscr{S}_{B}$. Moreover, any additional support must be contained in $\Sigma$.

As shown earlier, the contribution of $\mathscr{S}_{B}$ to $R_{N}^{10}$ is $\mathbb{Q}_{B} \oplus \mathcal{H}^{2}\left(\mathscr{I}_{\mathscr{C}}{ }_{B}\left(\Lambda_{B^{\circ}}^{8}\right)\right)$, where the second summand is supported on $\Delta$. In particular, this contribution restricted to $\Sigma \backslash \Delta$ is $\mathbb{Q}_{\Sigma \backslash \Delta}$, which does not yield the whole $R_{N \mid \Sigma \backslash \Delta}^{10}=\left(\mathbb{Q}_{\mid \Sigma \backslash \Delta} \oplus \mathscr{L}\right)(-5)$, as it is prescribed by Proposition 4.4.3.

It follows that $\Sigma$ is a support and that the local system of type $L$, defined on a suitable Zariski dense open subset of $\Sigma$, prescribed by Theorem 7.0.3 is the restriction of $\mathscr{L}$ to this open subset. It follows, also by virtue of Fact 5.7.1.(6), that the the associated Ngô string is $\mathscr{S}_{\Sigma}^{-}[-2](-1)$.

It also follows that the only other possible Ngô strings are supported at closed subvarieties of $\Delta$. Since $R_{N \mid \Delta}^{10} \simeq \mathbb{Q}_{\Delta}^{\oplus 2}(-5)$, Corollary 7.0.3 and Fact 5.7.1.(6) imply that the only remaining possible support is $\Delta$.

In particular, the only possible additional Ngô string is $S_{\Delta}^{\oplus \epsilon_{N}}$, with $\epsilon_{N}=0,1$.

By combining (85), the first equality in (91), and the sentence following (91), we see that $\epsilon_{N}=1$ iff $\epsilon_{B}^{2,8}=0$ iff $\epsilon_{\widetilde{M}}=1$. In particular, $\epsilon_{N}=\epsilon_{\widetilde{M}}$ and the r.h.s. of (87) follows, with the same value of $\epsilon$ on both sides.

The proposition is proved.

Remark 5.7.3. In analogy with (91), we note that the analysis carried out so-far implies that (85) can be improved to:

$$
\mathcal{H}^{8}\left(\mathscr{S}_{\Sigma}^{-}\right)=i_{\Sigma \backslash \Delta_{*}} \mathscr{L}
$$

\section{Proofs of Theorems A, B And B'}

We are now ready to combine the results proved so far with Theorem 7.0.3, the refined version of Ngô's Support Theorem. We prove Theorem A, then Theorem B and B'. The statement of Theorem B implies the statement of Theorem A. We chose however to first prove Theorem A and then Theorem B, as the extra layer given by the Hodge 
structures may make the topological arguments yielding Theorem A less clear. We then prove Theorem B, which implies Theorem B' thanks to a density argument using the period mapping.

\subsection{Proof of the main Theorem A.}

Fact 6.1.1. Let $\mathcal{A}$ be a semisimple Abelian category where every object has finite length and the isomorphism classes of simple objects form a set $\mathfrak{S}$. Recall that by definition the zero object is not simple. Every object $a \in \mathcal{A}$ is isomorphic to a unique finite direct sum of simple objects with multiplicities:

$$
a \simeq \bigoplus_{\mathfrak{s} \in \mathfrak{S}} \mathfrak{s}^{\oplus n_{\mathfrak{s}}(a)} .
$$

If we have an identity $[a]=[b]-[c]$ in the Grothendieck group $K(\mathcal{A})$, with $a, b, c \in \mathcal{A}$, then

$$
n_{\mathfrak{s}}(a)=n_{\mathfrak{s}}(b)-n_{\mathfrak{s}}(c) .
$$

Let $\phi: \operatorname{Obj}(\mathcal{A}) \rightarrow \mathfrak{M}$ be an assignment into a commutative group which is additive in exact sequences, then, if $[a]=[b]-[c]$ are as above, then

$$
\phi(a)=\phi(b)-\phi(c) .
$$

In the remainder of this section, we let $\mathbb{Q} G P P H S$ be the category of rational graded polarizable pure Hodge structures, we let $b_{*}$ denotes the graded dimension of the corresponding graded vector spaces (Betti numbers), and we let $h^{\bullet \star}$ denote the bi-graded dimension of the corresponding bi-graded vector spaces (Hodge numbers). If we set $\mathcal{A}=\mathbb{Q}$ GPPHS and $\phi=b_{*}, h^{\bullet \star}$, then we are in the situation of Fact 6.1 .1 , with $\mathfrak{M}=\mathbb{Z}^{\mathbb{N}},\left(\mathbb{Z}^{2}\right)^{\mathbb{N}}$, respectively.

Proposition 6.1.2. (Cut and paste of polarizable graded pure Hodge structures)

$$
\begin{gathered}
b_{*}(\widetilde{M})=b_{*}(N)+b_{*}\left(\left(\operatorname{Sym}^{2} M^{\prime}\right)^{\oplus 2}[-2]\right)-b_{*}\left(\left(M^{\prime 2}\right)[-2]\right)+b_{*}\left(\left(M^{\prime}\right)[-6]\right) ; \\
h^{\bullet \star}(\widetilde{M})=h^{\bullet \star}(N)+h^{\bullet \star}\left(\left(\operatorname{Sym}^{2} M^{\prime}\right)^{\oplus 2}[-2](-1)\right)-h^{\bullet \star}\left(\left(M^{\prime 2}\right)[-2](-1)\right)+h^{\bullet \star}\left(\left(M^{\prime}\right)[-6](-3)\right) .
\end{gathered}
$$

Proof. According to Proposition 5.7.2, to Remarks 5.1.2 and 7.0.5, we have isomorphisms of finite dimensional rational graded vector spaces, even of rational polarizable graded pure Hodge structures:

$$
\begin{array}{rlc}
H^{*}(\widetilde{M}) & \simeq & H^{*}\left(\mathscr{S}_{B}\right) \oplus H^{*}\left(\mathscr{S}_{\Sigma}^{+}\right)[-2](-1) \oplus H^{*}\left(\mathscr{S}_{\Delta}\right)^{\oplus 1+\epsilon}[-6](-3), \\
H^{*}(N) & \simeq & H^{*}\left(\mathscr{S}_{B}\right) \oplus H^{*}\left(\mathscr{S}_{\Sigma}^{-}\right)[-2](-1) \oplus H^{*}\left(\mathscr{S}_{\Delta}\right)^{\oplus \epsilon}[-6](-3), \\
H^{*}\left(M^{\prime 2}\right) & \simeq & H^{*}\left(\mathscr{S}_{\Sigma}^{+}\right) \oplus H^{*}\left(\mathscr{S}_{\Sigma}^{-}\right) .
\end{array}
$$


By working with the Abelian category of finite dimensional graded vector spaces, or even of rational polarizable graded pure Hodge structures, we obtain the identities in the corresponding Grothendieck groups: (note the important fact that the $\epsilon$ 's cancel out)

$$
H^{*}(\widetilde{M})=H^{*}(N)+\left(2 H^{*}\left(\operatorname{Sym}^{2} M^{\prime}\right)-H^{*}\left(M^{\prime 2}\right)\right)[-2](-1)+H^{*}\left(M^{\prime}\right)[-6](-3) .
$$

It remains to apply the identity (95).

Proof of Theorem A. It is enough to compute the Betti and Hodge number of $\widetilde{M}$.

The Betti and Hodge numbers of all the varieties appearing on the r.h.s. of (96) and (97), namely, of $N, M^{\prime}, M^{\prime 2}$, and of $\mathrm{Sym}^{2} M^{\prime}$ are known thanks to Göttsche's and Macdonald's formulae [Go-1990]. The odd Betti numbers of these varieties are zero, and their Hodge numbers in even degree are as follows. The Hodge numbers of $M^{\prime}$ :

$$
\begin{aligned}
& 1 \\
& \begin{array}{lll}
1 & 21 & 1
\end{array} \\
& \begin{array}{lllll}
1 & 21 & 232 & 21 & 1
\end{array}
\end{aligned}
$$

From the Hodge diamond of $M^{\prime}$, a direct computation gives the Hodge numbers of $M^{\prime 2}$ :

$$
\begin{aligned}
& 1 \\
& \begin{array}{ccccccccc} 
& & & 2 & 42 & 2 & & & \\
& & 3 & 84 & 907 & 84 & 3 & & \\
& 2 & 84 & 1350 & 9870 & 1350 & 84 & 2 & \\
1 & 42 & 907 & 9870 & 55596 & 9870 & 907 & 42 & 1 .
\end{array}
\end{aligned}
$$

and those of $\mathrm{Sym}^{2} M^{\prime}$ :

$$
\begin{array}{cccccccccc} 
& & & & 1 & & & & \\
& & & 1 & 21 & 1 & & & \\
& & 2 & 42 & 464 & 42 & 2 & & \\
& & 1 & 42 & 675 & 4935 & 675 & 42 & 1 & \\
1 & 21 & 464 & 4935 & 27914 & 4935 & 464 & 21 & 1 .
\end{array}
$$

The Hodge numbers of $N$ can be recovered from the generating series of [Go-1990] ${ }^{1}$ :

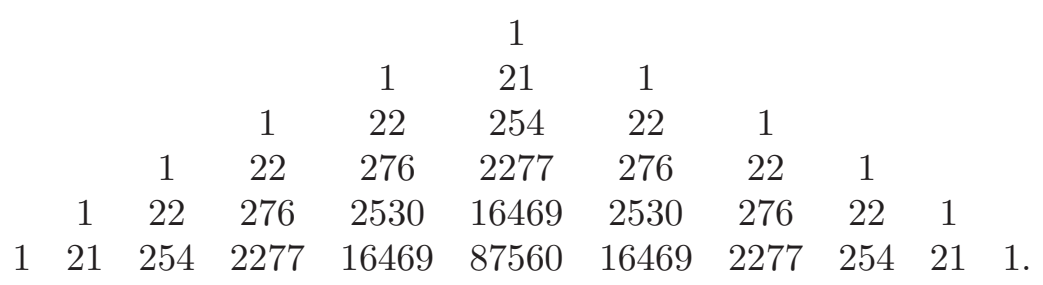

In view of (99), Theorem A now follows by direct calculation.

Remark 6.1.3. The proof of Theorem A combines the determination of the Ngo strings (Proposition 5.7.2) in $D^{b} M H M_{a l g}(B)$ with a cancelation occurring in a Grothendieck group (Proposition 6.1.2). J. Shen and Q. Yin have informed us that they can obtain the

\footnotetext{
${ }^{1}$ We used the script found at https://pbelmans.ncag.info/blog/2017/11/18/hodge-numbers-hilb/ to compute theses numbers.
} 
computation of the Hodge numbers as in Theorem A using the validity of Proposition 5.7.2 in $D^{b}(B, \mathbb{Q})$ (which can be considered the main technical result of this paper) and without invoking its validity at the level of mixed Hodge modules, and combine it with the main result in [Sh-Yi-2018]. This approach can then replace the last part of the proof of Theorem A given above.

\subsection{The main Theorem $\mathbf{B}^{\prime}$.}

Using Remarks 3.1.1 and 3.2.3 we can formulate the following, slightly more general, version of Theorem B:

Theorem $\mathbf{B}^{\prime}$. Let $(S, H)$ be a polarized $K 3$ surface of genus 2 . Let $w=(0,2 H, \chi), \chi$ odd, and $2 v^{\prime}=\left(0,2 H, 2 \chi^{\prime}\right)$ be Mukai vectors (cf. (15) and (16)) which are positive in the sense of Remark 3.1.1 . Let L, resp. $L^{\prime}$, be a polarization which is w-generic, resp. $2 v^{\prime}$-generic (cf. Remark 3.2.3). Finally, let $N=M_{w, L}(S)$ be the moduli space of L-stable sheaves on $S$ with Mukai vector $w$ and let $\widetilde{M}=\widetilde{M}_{2 v^{\prime}, L^{\prime}}(S)$ be a symplectic resolution of the moduli space $M_{2 v^{\prime}, L^{\prime}}(S)$ of $L^{\prime}$-semistable sheaves on $S$ with Mukai vector $2 v^{\prime}$. Then, using the notation as in the statement of Theorem B, we have isomorphisms:

$$
\begin{gathered}
\widetilde{M}=S^{(5)} \oplus\left[S^{(4)}\langle-1\rangle\right]^{\oplus 2} \oplus \mathbb{S}_{(2,2)}(S)\langle-1\rangle \oplus\left[S^{(3)}\langle-2\rangle\right]^{\oplus 2} \oplus \\
\oplus\left[\mathbb{S}_{(2,1)}(S)\langle-2\rangle\right]^{\oplus 2} \oplus[S \otimes S]\langle-3\rangle \oplus\left[S^{(2)}\langle-3\rangle\right]^{\oplus 3} \oplus[S\langle-4\rangle]^{\oplus 2} ; \\
N=S^{(5)} \oplus\left[S^{(3)} \otimes S\right]\langle-1\rangle \oplus\left[S \otimes S^{(2)}\right]^{\oplus 2}\langle-2\rangle \oplus\left[S^{2}\right]^{\oplus 2}\langle-3\rangle \oplus S\langle-4\rangle .
\end{gathered}
$$

The graded pure polarizable Hodge structures of all the varieties on the r.h.s. of (99) are known and can be expressed as follows in terms of the Hodge structure of the underlying K3 surface $S$. Let $V=H^{*}(S)$ be the rational Hodge structure of $S$. Using (19), Lemma 3.2.7, as well as [Go-So-1993] and MacDonald's formula, we can write:

$$
\begin{gathered}
H^{*}\left(M^{\prime}\right)=V^{(2)} \oplus V[-2](-1) \\
H^{*}\left(M^{\prime 2}\right)=V^{(2)} \otimes V^{(2)} \oplus 2 V^{(2)} \otimes V[-2](-1) \oplus V \otimes V[-4](-2) \\
H^{*}\left(\mathrm{Sym}^{2} M^{\prime}\right)=\mathrm{Sym}^{2} V^{(2)} \oplus V^{(2)} \otimes V[-2](-1) \oplus V^{(2)}[-4](-2) \\
H^{*}(N)=V^{(5)} \oplus V^{(3)} \otimes V[-2](-1) \oplus 2 V \otimes V^{(2)}[-4](-2) \oplus 2 V \otimes V[-6](-3) \oplus V[-8](-4) .
\end{gathered}
$$

For a partition $\lambda$ of an integer $k$, we denote by $\mathbb{S}_{(\lambda)}(-)$ the corresponding Schur functor. For the notation and basic properties of Schur functors we refer the reader to [Fu-Ha, Ch. 6]. Let $W$ be a vector space. By [Fu-Ha, Thm 6.3], the $G L(W)$-representation $W^{\otimes k}$ decomposes into a sum of irreducible subrepresentations, each of which is a Schur module $\mathbb{S}_{(\lambda)}(W)$. Moreover, if $W$ is a $\mathbb{Q}$-vector space, so are all of its Schur modules. It is well known that if $W$ is a $\mathbb{Q}$-vector space endowed with a polarizable Hodge structure, then $W^{\otimes k}$, as well as each of its irreducible representations, inherit compatible rational polarizable Hodge structures. This gives a rational polarizable Hodge structures on each of the Schur modules $\mathbb{S}_{(\lambda)}(W)$ which is such that the decomposition of $W^{\otimes k}$ into the direct sum of irreducible representations is also a decomposition into a direct sum of Hodge structures. 
In what follows, we need the following Lemma.

Lemma 6.2.1. The following isomorphisms of rational polarizable Hodge structures hold:

$$
\begin{aligned}
& V^{(2)} \otimes V=\mathbb{S}_{(2,1)}(V) \oplus V^{(3)} \\
& V^{(3)} \otimes V=\mathbb{S}_{(3,1)}(V) \oplus V^{(4)} \\
& V^{(2)} \otimes V^{(2)}=\mathbb{S}_{(2,2)}(V) \oplus \mathbb{S}_{(3,1)}(V) \oplus V^{(4)} \\
& \operatorname{Sym}^{2}\left(V^{(2)}\right)=\mathbb{S}_{(2,2)}(V) \oplus V^{(4)}
\end{aligned}
$$

Proof. By the observation on the compatibility of the Hodge structures that proceeds the lemma, it is enough to prove that these isomorphisms hold as $G L(V)$-subrepresentations $V^{(k)}$, for an appropriate integer $k$. The first two statements follow then from the formula on page 79 of [Fu-Ha], the second from the formula on page 81 of [Fu-Ha], while the last statement from [Fu-Ha, Ex. 6.16].

Using (107) and Lemma 6.2.1 we find the following isomorphism of rational polarizable Hodge structures:

$$
\begin{gathered}
H^{*}\left(M^{\prime 2}\right)=\underbrace{\mathbb{S}_{(2,2)}(V) \oplus \mathbb{S}_{(3,1)}(V) \oplus \mathbb{S}_{(4)}(V)}_{V^{(2)} \otimes V^{(2)}} \oplus \underbrace{V^{(3)}[-2](-1)^{\oplus 2} \oplus \mathbb{S}_{(2,1)}(V)[-2](-1)^{\oplus 2}}_{\left(V^{(2)} \otimes V[-2](-1)\right)^{\oplus 2}} \oplus V^{\otimes 2}[-4] . \\
H^{*}\left(\mathrm{Sym}^{2} M^{\prime}\right)=\underbrace{V^{(4)} \oplus \mathbb{S}_{(2,2)}(V)}_{\operatorname{Sym}^{2} V^{(2)}} \oplus \underbrace{V^{(3)}[-2](-1) \oplus \mathbb{S}_{(2,1}(V)[-2](-1)}_{V^{(2)} \otimes V[-2](-1)} \oplus V^{(2)}[-4](-2) \\
H^{*}(N)=V^{(5)} \oplus \underbrace{V^{(4)} \oplus S_{(3,1}(V)[-2](-1)}_{V^{(3)} \otimes V[-2](-1)} \oplus \underbrace{V^{(3)}[-4](-2) \oplus \mathbb{S}_{(2,1}(V)[-4](-2)}_{\left(V \otimes V^{(2)}[-4](-2)\right)^{\oplus 2}} \oplus \\
\oplus V \otimes V[-6](-3)^{\oplus 2} \oplus V[-8](-4) .
\end{gathered}
$$

Proof of Theorem $B^{\prime}$. We first prove the Theorem in the case when the degree two K3 surface $S$ is general in moduli, i.e., $S$ has Picard rank 1 (this is the statement of Theorem B). Then (99) holds. We thus substitute formulas (106),(108) (109) and (110), into equation (99) in order to express the Hodge structure of $\widetilde{M}$ in terms of that of $S$. Since the category of rational polarizable Hodge structures is semisimple, in view of Fact 6.1.1 we can make cancellations and find:

$$
\begin{aligned}
H^{*}(\widetilde{M}) & =V^{(5)} \oplus V^{(4)}[-2](-1)^{\oplus 2} \oplus \mathbb{S}_{(2,2)}(V)[-2](-1) \oplus V^{(3)}[-4](-2)^{\oplus 2} \oplus \mathbb{S}_{(2,1)}(V)[-4](-2)^{\oplus 2} \oplus \\
& \oplus V \otimes V[-6](-3) \oplus V^{(2)}[-6](-3)^{\oplus 3} \oplus V[-8](-4)^{\oplus 2} .
\end{aligned}
$$

This proves the theorem in case the degree two K3 surface $S$ has Picard rank 1.

Before proving the general case we recall that, since birational irreducible holomorphic symplectic manifolds have isomorphic integral Hodge structures, we can prove the theorem for any holomorphic symplectic birational model of $N$ or of $\widetilde{M}$. Thanks to Remark 3.2.3, it is thus sufficient to prove the statement for an arbitrary symplectic resolution of $M_{w, H}(S)$ or of $M_{2 v^{\prime}, H}(S)$. 
Let $\left(S_{0}, H_{0}\right)$ be a polarized K3 surface of genus 2. Choose a one-parameter deformation $(\mathcal{S}, \mathcal{H})$ of $\left(S_{0}, H_{0}\right)$ parameterized by a disk $\left(D, t_{0}\right)$ such that the subset of points $t \in D^{*}$ such that $N S\left(S_{t}\right)$ has rank one is dense. Let $v_{t}=\left(0,2 H_{t}, 2 \chi^{\prime}\right)$ be the corresponding Mukai vector. We consider the relative moduli space $\mathcal{M} \rightarrow D$, where $M_{t}=M_{v_{t}, H_{t}}\left(S_{t}\right)$. By [Pe-Ra-2013, Prop 2.20], up to shrinking $\left(D, t_{0}\right)$, we may assume that, for every $t \neq t_{0}$, the polarization $H_{t}$ is $v_{t}$-generic (cf. Remark 3.2.3). By [Pe-Ra-2013, Prop 2.23 and its proof], by blowing up the relative moduli space over the punctured disk $D^{*}$ along its singular locus results in a resolution $\widetilde{\mathcal{M}}_{D^{*}} \rightarrow \mathcal{M}_{D^{*}}$ which is point-by-point $t \in D^{*}$ a symplectic resolution. By Remark 3.2.3, $M_{t_{o}}$ admits a symplectic resolution $\widetilde{M}_{t_{0}} \rightarrow M_{t_{0}}$. By [Ko-La-Sa-Vo-2018, Thm 0.6, Cor 4.2], up to passing to a branched cover of $\left(D, t_{0}\right)$, we may assume that there is a smooth proper family of projective manifolds $\widetilde{M}_{D} \rightarrow D$ such that the restriction to $D^{*}$ coincides with the family $\widetilde{M}_{D^{*}} \rightarrow D^{*}$ introduced above and such that the central fiber is birational to $\widetilde{M}_{t_{0}}$.

Consider the two period mappings [Gr-1968] associated with the following two variations of (un-polarized) graded rational Hodge structures: the graded cohomology of the fibers of $\widetilde{M}_{D} \rightarrow D$ over $D$; the one on the r.h.s. of (1) as the K3 surface varies over $D$. By the first part of the proof, these two holomorphic period mappings coincide on the dense subset of $D$ seen above where the Néron-Severi of the K3 has rank one, so that the two mappings coincide on $D$.

\section{Appendix: Hodge-theoretic Ngô Strings}

The goal of this appendix is to state Theorem 7.0.3 (MHM Ngô strings over $\mathbb{C}$ ), which is a refinement of the Ngô Support Theorem [Ngô-2010, Théorème 7.2.1] valid in the Hodgetheoretic context of M. Saito's mixed Hodge modules [Sai-90, Sch-14]. We do not write out a detailed proof since, as it is explained below, it can obtained by repeating B.-C. Ngô's proof, with only two minor additional observations.

Theorem 7.0.3 admits several variants: $(a)$ varieties over finite fields and constructible $\overline{\mathbb{Q}}_{\ell}$-adic sheaves for the étale topology; varieties over the complex numbers and: $\left(b^{\prime}\right)$ constructible $\overline{\mathbb{Q}}_{\ell}$-sheaves for the étale topology; $\left(b^{\prime \prime}\right)$ constructible $\mathbb{Q}$-sheaves for the classical topology, with algebraic strata; $\left(b^{\prime \prime \prime}\right)$, i.e. Theorem 7.0.3 per se, mixed Hodge modules for the classical topology with algebraic strata. By the standard spreading out techniques of [Be-Be-De-1982][§6.1], version $(a)$ implies versions $\left(b^{\prime}, b^{\prime \prime}\right)$.

Ngô support Theorem [Ngô-2010, Théorème 7.2.1] is the (a)-version of (114), which roughly speaking, asserts that a support contributes a direct summand to the sheaf $R^{2 d} f_{*} \mathbb{Q}_{M}$. The paper [Ngô-2010] does not mention explicitly the complete collection of direct summands of $R f_{*} \overline{\mathbb{Q}}_{\ell M}$ on $S$ associated with a support $Z \subseteq S$. In fact, somewhat surprisingly, it seems a bit vague concerning this point; see [Ngô-2010, p.113, second sentence of last paragraph]. Even though this is not explicitly mentioned in [Ngô-2010], it is not difficult to make the Ngô Support Theorem more complete by making precise -building in an essential way on Ngô's method, especially [Ngô-2010, Proposition 7.4.10]all the direct summands supported on a given support appearing in the Decomposition Theorem for $R f_{*} \overline{\mathbb{Q}}_{\ell M}$. This leads to the versions $\left(a, b^{\prime}, b^{\prime \prime}\right)$ of Theorem 7.0.3. 
In this paper, we need Theorem 7.0.3, i.e. the Hodge-theoretic version $\left(b^{\prime \prime \prime}\right)$. Again, this is not difficult to achieve, by repeating B.-C. Ngô's proof of the support theorem by working in the categories $D^{b} M H M_{\text {alg }}(-)$. There are only two minor points to consider:

\section{Fact 7.0.1.}

(1) The weight argument [Ngô-2010, p.120-121] involving the $\overline{\mathbb{Q}}_{\ell}$-adic Tate module and Frobenius weights, goes through on the nose for the Tate module defined as an object in $D^{b} M H M_{a l g}(S)$ (cf. §2.2 and §2.1): we only need to work generically on a support, and the Tate module, being a direct image, is generically an admissible polarizable variation of mixed Hodge structures, of weights -1 and 0.

(2) The "Liberté" statement [Ngô-2010, Proposition 7.4.10]. B.-C. Ngô proves this by using finite fields. Since it is a topological statement concerning a graded module over a graded algebra in the category of lisse $\overline{\mathbb{Q}}_{\ell}$ sheaves in a geometric context, we can use the spreading out techniques mentioned above to carry over the "Liberté" statement from the algebraic closure of a finite field and graded semisimple lisse $\overline{\mathbb{Q}}_{\ell}$-sheaves, to the situation over the complex numbers and graded semisimple local systems. One then promotes the "Liberté" statement from the category of graded semisimple local systems, to the category of graded polarizable variations of pure Hodge structures by using the proof of [Ngô-2010, Lemma 7.4.11], where the graded object $E$ one obtains there is automatically a graded polarizable variation of pure Hodge structures.

Let us introduce the principal players in Theorem 7.0.3 by first giving the set-up and then by discussing some related objects.

Set-up 7.0.2. Let $(M, S, P)$ be a $\delta$-regular weak abelian fibration (Definition 2.2.1) of relative dimension $d:=\operatorname{dim}(M / S)=\operatorname{dim}(P / S)$, and such that $M / S$ is projective, $S$ is irreducible, and $M$ is a rational homology manifold $\left(\mathscr{I} \mathscr{C}_{M}=\mathbb{Q}_{M}\right)$.

We have assumed $\operatorname{dim}(M / S)=\operatorname{dim}(P / S)$ only to simplify the numerology. In this paper, we apply Theorem 7.0.3 to Lagrangian fibrations, so that the numerology gets simplified even further, since then one also has $d:=\operatorname{dim}(M / S)=\operatorname{dim}(P / S)=\operatorname{dim}(S)$.

Recall the notation fixed in $\S 2.1$. In particular, the supports of $R f_{*} \mathbb{Q}_{M}$ are the integral subvarieties of $S$ that support a non-zero direct summand of $R f_{*} \mathbb{Q}_{M}$. Let $\mathscr{A}$ be a finite set enumerating the supports of $R f_{*} \mathbb{Q}_{M}$, so that the supports are denoted $Z_{\alpha} \subseteq S, \alpha \in \mathscr{A}$. Let $g_{\alpha}: A_{\alpha} \rightarrow V_{\alpha}$ be the Abelian scheme (5) associated with $P_{\mid Z_{\alpha}}$, where $V_{\alpha} \subseteq Z_{\alpha}$ is a suitable Zariski open and dense subvariety contained in the regular locus of $Z_{\alpha}$, and we are free to shrink it if it is useful/necessary. Let $\delta_{\alpha}^{\text {ab }}$ be the relative dimension of $A_{\alpha} / V_{\alpha}$; then $\delta_{\alpha}^{\text {ab }}=d-\delta\left(Z_{\alpha}\right)$. Let $\Lambda_{\alpha}^{\bullet}:=R^{\bullet} g_{\alpha *} \mathbb{Q}_{A_{\alpha}}, 0 \leq \bullet \leq 2 \delta_{\alpha}^{\text {ab }}$; these are polarizable variations of pure Hodge structures of weight $\bullet$ on $V_{\alpha}$. Recall that a polarizable variation of pure Hodge structures $L$ of weight zero and of Hodge-Tate type on a nonsingular subvariety $V \subseteq S$ is determined by its underlying local system, and it gives rise to a pure Hodge module of weight $\operatorname{dim}(V)$ on the closure $Z$ of $V$, namely $I C_{Z}(L)$.

We can now state the main result of this appendix. 
Theorem 7.0.3. (MHM Ngô Strings Over $\mathbb{C}$ ) In the Set-up 7.0.2, there is an isomorphism in $D^{b} M H M_{a l g}(S)$ of pure objects of weight zero:

$$
R f_{*} \mathbb{Q}_{M} \simeq \bigoplus_{\alpha \in \mathscr{A}}\left\{\bigoplus_{\bullet=0}^{2 \delta_{\alpha}^{\mathrm{ab}}} \mathscr{I} \mathscr{C}_{Z_{\alpha}}\left(\Lambda_{\alpha}^{\bullet} \otimes L_{\alpha}\right)[-\bullet]\left[-2\left(d-\delta_{\alpha}^{\mathrm{ab}}\right)\right]\left(\delta_{\alpha}^{\mathrm{ab}}-d\right)\right\},
$$

where $L_{\alpha}$ is a polarizable variation of pure Hodge structures of weight zero, and of HodgeTate type, on $V_{\alpha}$ (we may need to shrink $V_{\alpha}$ to achieve this).

Moreover, we have:

(1) The identities:

$$
\delta_{\alpha}^{\mathrm{ab}}=\operatorname{dim}(M / S)-\operatorname{dim}(S)+\operatorname{dim}\left(Z_{\alpha}\right), \quad \forall \alpha \in \mathscr{A} .
$$

(2) Canonical isomorphisms:

$$
\Lambda_{A_{\alpha}}^{\delta_{\alpha}^{\mathrm{ab}}-\bullet}=\Lambda_{A_{\alpha}}^{\delta_{\alpha}^{\mathrm{ab}}+\bullet}(\bullet), \quad \Lambda_{A_{\alpha}}^{\delta_{\alpha}^{\mathrm{ab}}-\bullet} \otimes L_{\alpha}=\Lambda_{A_{\alpha}}^{\delta_{\alpha}^{\mathrm{ab}}+\bullet}(\bullet) \otimes L_{\alpha}, \quad \forall 0 \leq \bullet \leq \delta_{\alpha}^{\mathrm{ab}} .
$$

In addition, one can realize other such isomorphisms, with associated Primitive Lefschetz Decompositions, via the Relative Hard Lefschetz Theorem for the projective morphisms $g_{\alpha}$.

(3) The object $R^{2 d} f_{*} \mathbb{Q}_{M}$ on $S$ admits the following pure direct summand, where $i_{V_{\alpha}}$ : $V_{\alpha} \rightarrow Z_{\alpha}$ is the natural open immersion, and the equalities are summand-bysummand:

$$
\bigoplus_{\alpha} i_{V_{\alpha *}} L_{\alpha}(-d)=\bigoplus_{\alpha} \mathscr{I} \mathscr{C}_{Z_{\alpha}}\left(L_{\alpha}(-d)\right)
$$

If the general fiber of $M / S$ is integral and if we take $Z=S$, with $S$ normal, then $i_{V_{S} *} L=\mathbb{Q}_{S}$.

If all the fibers of $M / S$ are integral, then the only support is $S$ and, if $S$ is normal, then $i_{V_{S *}} L=\mathbb{Q}_{S}$.

(4) By taking degree * cohomology in (111), we have an isomorphism in the category of graded polarizable mixed Hodge structures (polarizable pure Hodge structures if $S$, hence $M$, is complete):

$$
\left.H^{\star}(M, \mathbb{Q}) \simeq \bigoplus_{\alpha}\left\{\bigoplus_{b=0}^{2 \delta_{\alpha}^{\mathrm{ab}}} I H^{\star-b-2 d+2 \delta_{\alpha}^{\mathrm{ab}}}\left(Z_{\alpha}, \Lambda_{\alpha}^{b} \otimes L_{\alpha}\right)\right)\left(\delta_{\alpha}^{\mathrm{ab}}-d\right)\right\}, \quad \forall \star .
$$

Proof. Recall that $d:=\operatorname{dim}(M / S)=\delta\left(Z_{\alpha}\right)+\delta_{\alpha}^{\text {ab }}$. The identity (112) follows by combining the inequality in [Ngô-2010, Proposition 7.2.2] (which is stated and proved in the étale context over the closure of an algebraically closed field, and, by [Be-Be-De-1982][§6.1], remains valid for the classical topology over $\mathbb{C}$ ) taken together with the $\delta$-regularity hypothesis, which is precisely the opposite inequality. This proves (1)

Note that (2) is a statement about polarizable variations of pure Hodge structures (pvphs) The first canonical isomorphism in (113) is standard and due to Lieberman (see [Ngô-2010, §7.4.4]). Clearly, the second one follows from the first by twisting by any pvphs. The remaining statements in (2), concerning the Hard Lefschetz Theorem, are also standard. This proves (2). 
We are left with proving (111) and (3) and (4). Recall that we are free to shrink the Zariski-dense open subset $V_{\alpha} \subseteq Z_{\alpha}$ if useful/necessary. In what follows, we do so to meet our needs without explicit mention.

The Decomposition Theorem in $D^{b} M H M_{a l g}(S)$ for the projective morphism $f: M \rightarrow S$ implies that there is an isomorphism

$$
R f_{*} \mathbb{Q}_{M} \simeq \bigoplus_{\alpha \in \mathscr{A}}\left(\bigoplus_{i \in o_{\alpha}^{-}}^{o_{\alpha}^{+}} \mathscr{I} C_{Z_{\alpha}}\left(\mathcal{K}_{\alpha}^{i}\right)[-i],\right)
$$

where the $\mathcal{K}_{\alpha}^{i}$ are pvphs of weight $i$ on $V_{\alpha}$, and where $o_{\alpha}^{-} \geq 0$ marks the first occurrence of a non-zero direct summand supported on $Z_{\alpha}$, and $o_{\alpha}^{+} \geq o_{\alpha}^{-}$marks the last.

We fix $\alpha \in \mathscr{A}$.

The graded object $\mathcal{K}_{\alpha}:=\oplus_{i \geq 0} \mathcal{K}_{\alpha}^{i}$ lives in degrees in the interval $\left[o_{\alpha}^{-}, o_{\alpha}^{+}\right]$, and has graded weight zero, i.e. weight $i$ in degree $i$.

By [Ngô-2010][Proposition 7.2.2], which (by [Be-Be-De-1982][§6.1], again), remains valid in $D^{b}(S, \mathbb{Q})$, the local system $\mathcal{K}_{\alpha}^{o_{\alpha}^{+}}$is a direct summand of $\left(R^{2 d} f_{*} \mathbb{Q}_{M}\right)_{\mid V_{\alpha}}$. It follows that $o_{\alpha}^{+}=2 d$; in particular, the last occurrence $o_{\alpha}^{+}$is independent of $\alpha$.

By combining (116) with the symmetries stemming from Verdier Duality, we deduce easily that $o_{\alpha}^{-}=2 d-2 \delta_{\alpha}^{\mathrm{ab}}$, i.e. the interval $\left[o_{\alpha}^{-}, o_{\alpha}^{+}\right]=\left[2 d-2 \delta_{\alpha}^{\mathrm{ab}}, 2 d\right]$ has length twice the relative dimension $\delta_{\alpha}^{\text {ab }}$ of the abelian scheme $A_{\alpha} / V_{\alpha}$.

The graded object

$$
\wedge_{\alpha}=\bigoplus_{i=-2 \delta_{\alpha}^{\mathrm{ab}}}^{0} \wedge_{\alpha}^{i}:=\bigoplus_{i=-2 \delta_{\alpha}^{\mathrm{ab}}}^{0} R^{2 \delta_{\alpha}^{\mathrm{ab}}+i} g_{\alpha *} \mathbb{Q}_{A_{V_{\alpha}}}\left(\delta_{\alpha}^{\mathrm{ab}}\right):
$$

is concentrated in semi-negative degrees in the interval $\left[-2 \delta_{\alpha}^{\mathrm{ab}}, 0\right]$; has weight zero (seminegative weight $i$ in degree $i$ ); has, via Poincaré Duality, as stalks the homology of the fibers of $A_{\alpha} / V_{\alpha}$; it admits, via the Pontryagin product operation, a natural structure of graded algebra $\wedge_{\alpha}^{i} \otimes \wedge_{\alpha}^{j} \rightarrow \wedge_{\alpha}^{i+j}$.

The graded object of pure weight zero

$$
\Lambda_{\alpha}:=\bigoplus_{i=0}^{2 \delta_{\alpha}^{\mathrm{ab}}} \Lambda_{\alpha}^{i}:=\bigoplus_{i=0}^{2 \delta_{\alpha}^{\mathrm{ab}}} R^{i} g_{\alpha *} \mathbb{Q}_{A_{\alpha}}
$$

is a free graded $\wedge_{\alpha}$-module generated in degree $2 \delta_{\alpha}^{\mathrm{ab}}$ by $\Lambda_{\alpha}^{2 \delta_{\alpha}^{\mathrm{ab}}} \simeq \mathbb{Q}_{V_{\alpha}}\left(-\delta_{\alpha}^{\mathrm{ab}}\right)$, via the Pontryagin product operation which, again via Poincaré Duality, is expressed by operations (recall that $\star \leq 0$ and $\bullet \geq 0$ )

$$
\Lambda_{\alpha}^{\star} \otimes \Lambda_{\alpha}^{\bullet} \rightarrow \Lambda_{\alpha}^{\bullet+\star}
$$

By [Ngô-2010][Proposition 7.4.10], we have that $\mathcal{K}_{\alpha}$ is a free graded $\wedge_{\alpha}$-module. In fact, to be precise, we need to make sure that the action $\wedge_{\alpha}$ on $\mathcal{K}_{\alpha}$ (cf. [Ngô-2010][pp. 120-121] is defined and free in the category of pvphs on $V_{\alpha}$; for this we use Fact 7.0.1, parts (1) (action) and (2) (freeness). 
Since $\mathcal{K}_{\alpha}$ has non trivial entries only in the interval $\left[2 d-2 \delta_{\alpha}^{\mathrm{ab}}, 2 d\right]$, which has length $2 \delta_{\alpha}^{\mathrm{ab}}$, we deduce that, as a graded object, we have $\mathcal{K}_{\alpha}=\left(\Lambda_{\alpha} \otimes L_{\alpha}\right)\left[-2\left(d-\delta_{\alpha}^{\mathrm{ab}}\right)\right]$, where $L_{\alpha}:=\mathcal{K}_{\alpha}^{2 d}\left(\delta_{\alpha}^{\mathrm{ab}}\right)$.

At this point, by taking care of the bookkeeping of weights and Tate shifts, (111) follows, and $L_{\alpha}$ is as predicated since $L_{\alpha}(-d)$ is a pvphs direct summand of the pvphs $\left(R^{2 d} f_{*} \mathbb{Q}_{M}\right)_{\mid V_{\alpha}}$ which, having stalks generated by the fundamental classes of the irreducible components of the fibers, is pure, of Hodge-Tate type with weights $2 d$.

At this point, (3) and (4) follows easily: the latter by taking cohomology; the former by inspecting the contributions of each support to the top cohomology sheaf $R^{2 d} f_{*} \mathbb{Q}_{M}$, and recalling that normality implies uni-branch, and this latter implies that the sheaf-theoretic direct image of the constant sheaf from a Zariski-dense open is also constant.

Definition 7.0.4. We call the $\alpha$-summands in (111) the $N g \hat{o}$ strings of $R f_{*} \mathbb{Q}_{M}$. If we set:

$$
\mathscr{S}_{\alpha}:=\bigoplus_{b=0}^{2 \delta_{\alpha}^{\mathrm{a}}} \mathscr{I}_{C_{Z_{\alpha}}}\left(\Lambda_{\alpha}^{b} \otimes L_{\alpha}\right)[-b],
$$

then we may re-write (111) as follows:

$$
R f_{*} \mathbb{Q}_{M} \simeq \bigoplus_{\alpha} \mathscr{S}_{\alpha}\left[-2\left(d-\delta_{\alpha}^{\mathrm{ab}}\right)\right]\left(\delta_{\alpha}^{\mathrm{ab}}-d\right) .
$$

We also call the $\mathscr{S}_{\alpha}$ 's the $N g \hat{o}$ strings of $R f_{*} \mathbb{Q}_{M}$; these start in cohomological degree zero and are pure of weight zero.

Each Ngô string in (111) is pure of weight zero and a direct sum of a collection of intersection complexes supported precisely at $Z_{\alpha}$, placed in cohomological degree in the interval $\left[2 d-2 \delta_{\alpha}^{\mathrm{ab}}, 2 d\right]$. Up to the twist by $L_{\alpha}$, the coefficients of the term indexed by $\bullet$ are the direct image $\Lambda_{\alpha}^{\bullet}=R^{b} g_{\alpha *} \mathbb{Q}_{A_{\alpha}}$. These latter direct images on $V_{\alpha}$ depend on the group scheme $P / S$, not on $M / S$. We record this fact in the following Remark which plays an important role in our proof of our main Theorems A and B, where it is crucial to be able to relate the Decomposition Theorem for the $\delta$-regular weak abelian fibration structure on $\widetilde{M}$, to the Decomposition Theorems for the auxiliary $\delta$-regular weak abelian fibrations we consider; see Lemmata 5.4.1 and 5.5.1, and Propositions 5.6.1 and 5.7.2.

Remark 7.0.5. As an object in $D^{b} M H M_{a l g}(S)$, the Ngô string $\mathscr{S}_{\alpha}$ associated with the support $Z_{\alpha}$ depends only on the Abelian group scheme $A_{\alpha}$ and on the topological local system $L_{\alpha}$ (because it can be enriched uniquely to a polarizable variation of Hodge structures of Hodge-Tate type and weight zero). The same holds for the graded polarizable mixed Hodge structure (pure and polarizable if $Z_{\alpha}$ is complete) $H^{\star}\left(Z_{\alpha}, \mathscr{S}_{\alpha}\right)$. Clearly, the $L_{\alpha}$ 's do depend on $M / S$.

\section{REFERENCES}

[EGA IV(3)] A. Grothendieck, Éléments de géométrie algébrique. IV. Étude locale des schémas et des morphismes de schémas. III. Inst. Hautes Études Sci. Publ. Math. No. 281966255 pp. 19

[EGA IV(4)] A. Grothendieck, Éléments de géométrie algébrique. IV. Étude locale des schémas et des morphismes de schémas. IV. Inst. Hautes Études Sci. Publ. Math. No. 321967361 pp. 7 
[SGA 4.3] P. Deligne M. Artin, A. Grothendieck; J.L.Verdier, Séminaire de Géométrie Algébrique du Bois Marie - 1963-64 - Théorie des topos et cohomologie étale des schémas - (SGA 4) - vol. 3. Lecture Notes in Mathematics. 305. Berlin; New York: Springer-Verlag. pp. vi+640. 37, 38

[Al-Kl-1980] A. B. Altman, S. L. Kleiman, "Compactifying the Picard scheme," Adv. in Math. 35 (1980), no. $1,50-112.19$

[Ar-Sa-2018] E. Arbarello, G. Saccà, "Singularities of moduli spaces of sheaves on K3 surfaces and Nakajima quiver varieties", Adv. in Math. 329 (2018) 649-703. 15

[Ba-Ma-2014a] A. Bayer, E. Macrì, "MMP for moduli of sheaves on K3's via wall-crossing: nef and movable cones, Lagrangian fibrations," Invent. Math. 198 (2014), no. 3, 505-590. 15

[Ba-Ma-2014b] A. Bayer, E. Macrì. "Projectivity and birational geometry of Bridgeland moduli spaces," J. Amer. Math. Soc. 27 (2014), no. 3, 707-752. 13

[Be-Be-De-1982] Faisceaux pervers, Analysis and topology on singular spaces, I (Luminy, 1981), 5-171, Astérisque, 100, Soc. Math. France, Paris, 1982. 54, 56, 57

[Bea-1983] A. Beauville, "Variétés Kähleriennes dont la première classe de Chern est nulle," J. Differential Geom. 18 (1983), no. 4, 755-782 (1984). 2

[Bea-1991] A. Beauville, "Systèmes hamiltoniens complètement intégrables associés aux surfaces K3," Symposia Mathematica 32, 25-31; Academic Press (1991). 16

[Bea-1999] A. Beauville, "Counting rational curves on K3 surfaces," Duke Math. J. 97 (1999), no. 1, 99-108. 9, 18, 27

[Bo-1973] F. A. Bogomolov, "The decomposition of Kähler manifolds with a trivial canonical class." (Russian) Mat. Sb. (N.S.) 93(135) (1974), 573-575, 630. 2

[Bo-1991] A. Borel, Linear Algebraic Groups, Second Enlarged Edition, Springer-Verlag, 1991. 12

[Bo-Lu-Ra-1990] S. Bosch, W. Lütkebohmert, M. Raynaud, Néron models. Ergebnisse der Mathematik und ihrer Grenzgebiete (3) [Results in Mathematics and Related Areas (3)], 21. Springer-Verlag, Berlin, 1990. 13, 19, 25

[Bu] A. Buium, "Sur le nombre de Picard des revêtements doubles des surfaces algébriques." C. R. Acad. Sci. Paris Sér. I Math. 296 (1983), no. 8, 361-364. 16

[Ch-Ka-2016] D. Chen, J. Kass, "Moduli of generalized line bundles on a ribbon," J. Pure Appl. Algebra 220 (2016), no. 2, 822-844. 36

[Ch-2002] X. Chen, "A simple proof that rational curves on K3 are nodal," Math. Ann. 324 (2002), no. 1, 71-104. 16

[Co-1993] P. R. Cook, "Local and Global Aspects of the Module Theory of Singular Curve", PhD. Thesis, University of Liverpool, 1993. 27, 28

[de-Mi-2000] M.A. de Cataldo, L. Migliorini, "The Douady space of a complex surface," Adv. Math. 151 (2000), no. 2, 283-312. 2

[de-Mi-2002] M.A. de Cataldo, L. Migliorini, "The Chow groups and the motive of the Hilbert scheme of points on a surface," J. Algebra 251 (2002), no. 2, 824-848. 2

[de-2017] M.A. de Cataldo, "A support theorem for the Hitchin fibration: the case of $S L_{n}$," Compos. Math. 153 (2017), no. 6, 1316-1347. 21

[de-Mi-2009] M.A. de Cataldo, L. Migliorini, "The Decomposition Theorem, perverse sheaves and the topology of algebraic maps," Bull. Amer. Math. Soc. (N.S.) 46 (2009), no. 4, 535-633. 5, 6, 7, 23

[de-2012] M.A. de Cataldo, "The perverse filtration and the Lefschetz Hyperplane Theorem, II," J. Algebraic Geometry 21 (2012) 305-345. 7

[de-PCMI] M.A. de Cataldo, "Perverse sheaves and the topology of algebraic varieties," Geometry of moduli spaces and representation theory, 1-58, IAS/Park City Math. Ser., 24, Amer. Math. Soc., Providence, RI, 2017. 43, 47

[DS-1979] C. D'Souza, "Compactification of generalised Jacobians," Proc. Indian. Acad. Sci. Vol. 88 A, Part III, n.5, 1979, pp.419-457. 27

[Fl-Fu-Zh-2019] S. Floccari, L. Fu, and Z. Zhang, "On the motive of O'Grady's ten-dimensional hyperKähler varieties," arXiv:1911.06572, 20195 
[Fu-1983] A. Fujiki “On primitively symplectic compact Kähler V-manifolds of dimension four," Classification of algebraic and analytic manifolds (Katata 1982), Progr. Math. 39, 1983, Birkhäuser, pp.71-250. 2

[Fu-Ha] W. Fulton, J. Harris, "Representation theory," Graduate Texts in Mathematics, 129, SpringerVerlag, New York, 1991. 4, 52, 53

[Gi-1977] D. Gieseker, "On the moduli of vector bundles on an algebraic surface," Ann. of Math. (2) 106 (1977), no. 1, 45-60. 13

[Go-1990] L. Göttsche, "The Betti numbers of the Hilbert scheme of points on a smooth projective surface," Math. Ann. 286 (1990), no. 1-3, 193-207. 2, 51

[Go-So-1993] L. Göttsche, W. Soergel, "Perverse sheaves and the cohomology of Hilbert schemes of smooth algebraic surfaces," Math. Ann. 296 (1993), no. 2, 235-245. 2, 38, 52

[Gr-1968] P. Griffiths, "Periods of integrals on algebraic manifolds,I,II" Amer. J. Math. 90, 1968, pp. $568-626,805-865.54$

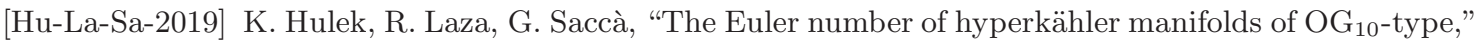
arXiv:1902.00781. 2, 3

[Hu-2003] D. Huybrechts, "The Kähler cone of a compact hyperkähler manifold," Math. Ann. 326 (2003), no. $3,499-513.18$

[Hu-Le-1997] D. Huybrechts and M. Lehn. The geometry of moduli spaces of sheaves. Number E 31 in Aspects of Mathematics. Vieweg, 1997. 13, 14, 15, 19, 27, 28, 35

[Ka-Le-2007] Kaledin, D. and Lehn, M. "Local structure of hyper-Kähler singularities in O'Grady's examples," Mosc. Math. J., 7(4):653-672, 766-767, 2007. 14

[Ka-Le-So-2006] Kaledin, D. and Lehn, M. and Sorger, Ch. "Singular symplectic moduli spaces," Invent. Math., 164(3):591-614, 2006. 13, 14

[Ko-Mo-1998] J. Kollár, S. Mori, Birational geometry of algebraic varieties. Cambridge Tracts in Mathematics, 134. Cambridge University Press, Cambridge, 1998. 17

[Ko-La-Sa-Vo-2018] J. Kollár, R. Laza, G. Saccà, C. Voisin, "Remarks on degenerations of hyper-Kähler manifolds," to appear in Ann. Inst. Four. 3, 54

[Le-So-2006] M. Lehn, C. Sorger, "La singularité de O’Grady," J. Algebraic Geom., 15(4):753-770, 2006. $14,15,23,38$

[G-K-L-R-2019] M. Green, Y.-J. Kim, R. Laza, C. Robles "The LLV decomposition of hyper-Kaehler cohomology," arXiv:1906.03432. 5

[LSV-2017] R. Laza, G. Saccà, and C. Voisin, "A hyper-Kähler compactification of the intermediate Jacobian fibration associated with a cubic 4-fold," Acta Math. 218 (2017), no. 1, 55-135. 3

[LP-1993a] J. Le Potier, "Faisceaux semi-stables de dimension 1 sur le plan projectif," Rev. Roumaine Math. Pures Appl., 38(7-8):635-678, 1993. 14, 16, 25

[LP-1993b] J. Le Potier, Systèmes cohérents et structures de niveau. Astérisque No. 214 (1993). 16

[Lo-Lu-1997] E. Looijenga, V.A. Lunts, "A Lie algebra attached to a projective variety," Invent. Math. 129 (1997), no. 2, 361-12. 5

[Ma-2000] D. Matsushita, "Equidimensionality of Lagrangian fibrations on holomorphic symplectic manifolds," Math. Res. Lett. 7 (2000), no. 4, 389-391. 9, 16, 17

[Ma-1986] H. Matsumura, Commutative Ring Theory, Translated from the Japanese by M. Reid. Cambridge Studies in Advanced Mathematics, 8. Cambridge University Press, Cambridge, 1986. 17

[Me-Ra-Vi-2017] M. Melo, A. Rapagnetta, F. Viviani, "Fine compactified Jacobians of reduced curves", Trans. Amer. Math. Soc. 369 (2017), no. 8, 5341-5402. 25

[Me-Zh-2016] C. Meachan, Z. Zhang, "Birational geometry of singular moduli spaces of O'Grady type", Adv. Math. 296 (2016), 210-267. 15

[Mi-2012] J.S Milne, Basic Theory of Affine Group Schemes, online 2012 notes. 7

[Mo-Ra-Sa-2018] G. Mongardi, A. Rapagnetta, and G. Saccà, “The Hodge diamond of O'Grady's sixdimensional example," Compos. Math. 154 (2018), no. 5, 984-1013. 2

[Mo-2007] S. Mozgovyy, "The Euler number of O'Gradys 10-dimensional symplectic manifold," Ph.D. thesis, Johannes Gutenberg-Universität Mainz, 2007. 2, 3, 28, 29, 30 
[Mu-1984] S. Mukai. "Symplectic structure of the moduli space of sheaves on an abelian or $K 3$ surface," Invent. Math., 77(1):101-116, 1984. 13, 16

$[\mathrm{Mu}]$ D. Mumford, J. Fogarty, F. Kirwan, Geometric invariant Theory, Third edition. Ergebnisse der Mathematik und ihrer Grenzgebiete (2) [Results in Mathematics and Related Areas (2)], 34. SpringerVerlag, Berlin, 1994. 36

[Na-Ra-1969] M.S. Narasimhan, S. Ramanan, "Moduli of vector bundles on a compact Riemann surface," Ann. of Math. (2) 891969 14-51. 27

[Ngô-2010] B.-C. Ngô, "Le lemme fondamental pour les algebres de Lie," Publ. Math. Inst. Hautes Études Sci. No. 111 (2010), 1-169. 7, 8, 9, 54, 55, 56, 57

[Ngô-2008] B.-C. Ngô, "Le lemme fondamental pour les algebres de Lie," arXiv: 0801.0446v1. 37

[Ngô-2011] B.-C. Ngô, "Decomposition Theorem and abelian fibration. On the stabilization of the trace formula," 253-264, Stab. Trace Formula Shimura Var. Arith. Appl., 1, Int. Press, Somerville, MA, 2011. 7

[O'G-1999a] K. G. O'Grady, "Desingularized moduli spaces of sheaves on a K3," J. Reine Angew. Math. 512 (1999), 49-117. 2, 13, 14

[O'G-1999b] K. G. O'Grady, “A new six-dimensional irreducible symplectic variety," J. Algebraic Geom. 12 (2003), no. 3, 435-505. 2

[Pe-Ra-2017] A. Perego, A. Rapagnetta, "The moduli spaces of sheaves on $K 3$ surfaces are irreducible symplectic varieties," arXiv:1802.01182. 13

[Pe-Ra-2013] A. Perego, A. Rapagnetta, "Deformation of the O'Grady moduli spaces," J. Reine Angew. Math. 678 (2013), 1-34. 14, 15, 54

[Ra-2008] A. Rapagnetta, "On the Beauville form of the known irreducible symplectic varieties." Math. Ann. 340 (2008), no. 1, 77-95. 2, 14, 24, 39

[Re-1980] C. J. Rego, "The compactified Jacobian," Annales scientifiques de l' E.N.S. 4e série, tome 13, no. 2 (1980), 211-223. 16, 18, 20, 24, 25, 45

[Re-1982] C. J. Rego, C. J. "Compactification of the space of vector bundles on a singular curve," Comment. Math. Helv. 57 (1982), no. 2, 226-236. 27

[SD-1972] B. Saint-Donat, "Projective models of K3 surfaces," Amer. J. Math. 96 (1974), 602-639. 16

[Sai-90] M. Saito, "Mixed Hodge modules," Publ. Res. Inst. Math. Sci. 26 (1990), no. 2, 221-333. 5, 6, 54

[Sch-14] C. Schnell, “An overview of Morihiko Saito's theory of mixed Hodge modules," arXiv:1405.3096. 5,54

[Se-1982] C.S. Seshadri, Fibrés vectoriels sur les courbes algébriques. (French) [Vector bundles over algebraic curves] Notes written by J.-M. Drezet from a course at the École Normale Supérieure, June 1980. Astérisque, 96. Société Mathématique de France, Paris, 1982. 209 pp. 27

[Sh-Yi-2018] J. Shen, Q. Yin, "Topology of lagrangian fibrations and Hodge theory of hyperkahelr manifolds," arXiv:1812.10673. 52

[Ve-96] M. Verbitsky, "Cohomology of compact hyper-Kähler manifolds and its applications", Geom. Funct. Anal. 6 (1996), no. 4, 601-611. 5

[Yos-01] K. Yoshioka. "Moduli spaces of stable sheaves on abelian surfaces," Math. Ann., 321(4):817-884, 2001. 13, 14, 15

Mark Andrea A. de Cataldo, Stony Brook University

Email address: mark.decataldo@stonybrook.edu

Antonio Rapagnetta, Università di Roma 2

Email address: rapagnet@axp.mat.uniroma2.it

Giulia Saccì, Columbia University

Email address: gs3032@columbia.edu 